\title{
Development of a High-Temperature Two-Component Explosive for Geothermal Stimulation
}

\section{A STATUS REVDEW}

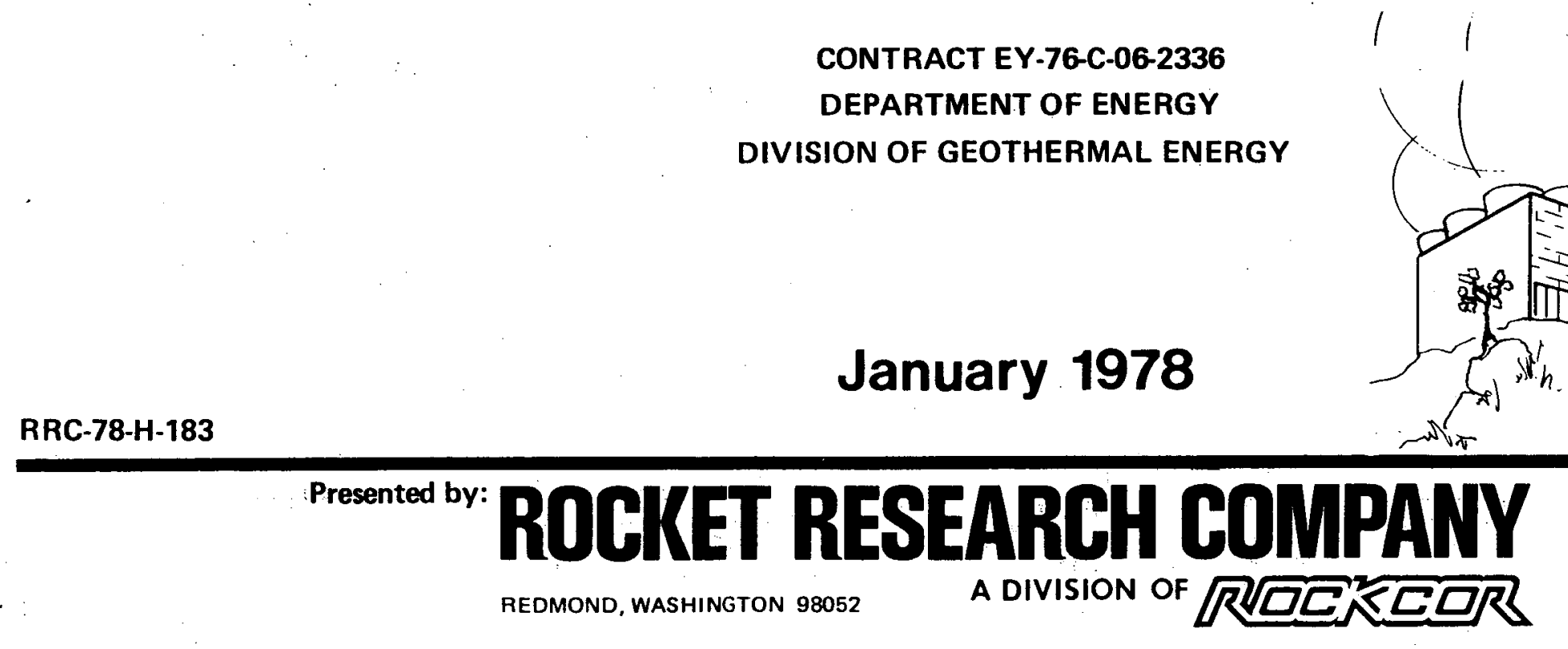




\section{DISCLAIMER}

This report was prepared as an account of work sponsored by an agency of the United States Government. Neither the United States Government nor any agency Thereof, nor any of their employees, makes any warranty, express or implied, or assumes any legal liability or responsibility for the accuracy, completeness, or usefulness of any information, apparatus, product, or process disclosed, or represents that its use would not infringe privately owned rights. Reference herein to any specific commercial product, process, or service by trade name, trademark, manufacturer, or otherwise does not necessarily constitute or imply its endorsement, recommendation, or favoring by the United States Government or any agency thereof. The views and opinions of authors expressed herein do not necessarily state or reflect those of the United States Government or any agency thereof. 


\section{DISCLAIMER}

Portions of this document may be illegible in electronic image products. Images are produced from the best available original document. 


\section{CORPORATE STRUCTURE}

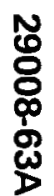

ROCKCOR, INC.

REDMOND, WASH.

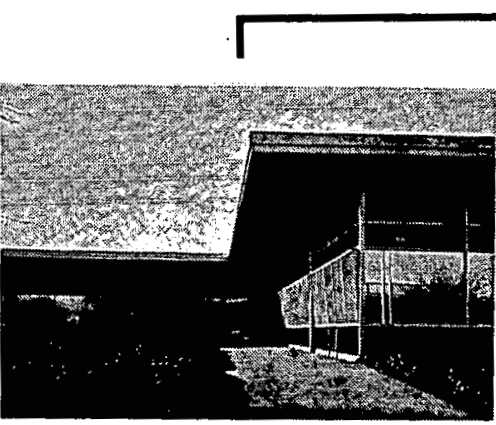

LARSE CORPORATION PALO ALTO, CALIF.

- TELEPHONE DIAL OFFICE FAULT REPORTING AND CONTROL SYSTEMS

- microwave Radio fault REPORTING AND CONTROL SYSTEMS

- fire aNd buRglar alarm comMUNICATION SYSTEMS

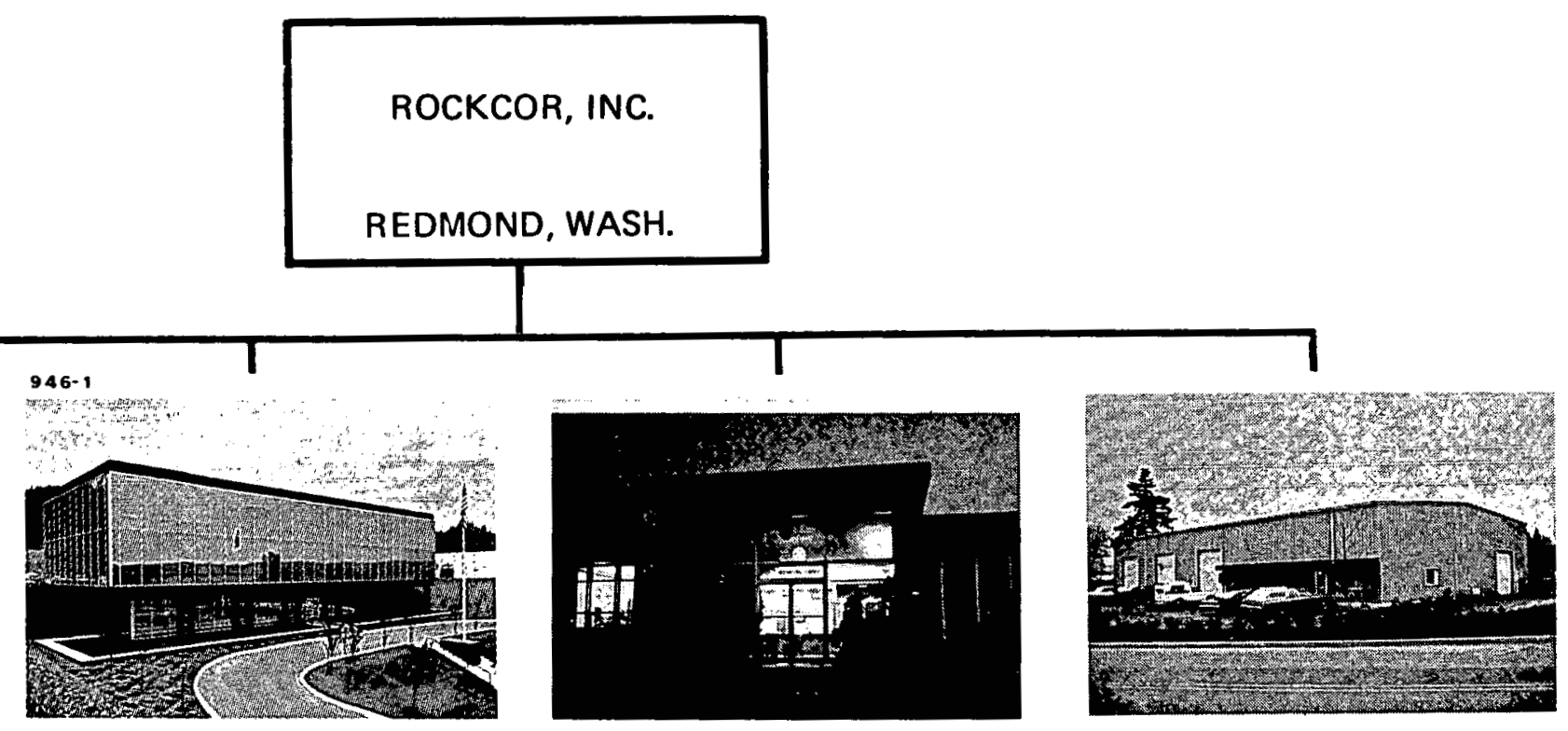

ROCKET RESEARCH COMPANY REDMOND, WA.

- ROCKETENGINES AND PROPULSION SYSTEMS

- gAS GENERATORS

- aUtomotive pRodUcts

- chemical technology

- ENERGY R\&D

- manufacturing aND TEST SERVICES

- LICENSOR For ASTROLITE EXPLOSIVES

- LICENSOR for CIRCUIT BOARD STAMPING TECHNOLOGY
PHYSICS INTERNATIONAL COMPANY

SAN LEANDRO, CALIF.

- PULSED POWER SYSTEMS

- nUCLEAR weAPONS EFFECTS SIMULATORS

- NUCLEAR WEAPONS EFFECTS TESTING AND ANALYSIS

- CONVENTIONAL EXPLOSIVES ENGINEERING

- COMPUTER CODES

- pIEZOELECTRIC ACTUATORS

- thermonUCLEAR fUSION POWER RESEARCH

\section{PETROLEUM TECHNOLOGY} CORPORATION REDMOND, WA.

- OIL AND GAS WELL EXPLOSIVE STIMULATION

- UNDERGROUND EXPLOSIVE FRACTURING FOR RESOURCE EXTRACTION 
ROCKET RESEARCH COMPANY

YORK CENTER FACILITY, REDMOND, WASHINGTON

$\overrightarrow{\vec{D}}$
$\stackrel{\leftrightarrow}{\mathbf{W}}$
$\dot{\infty}$

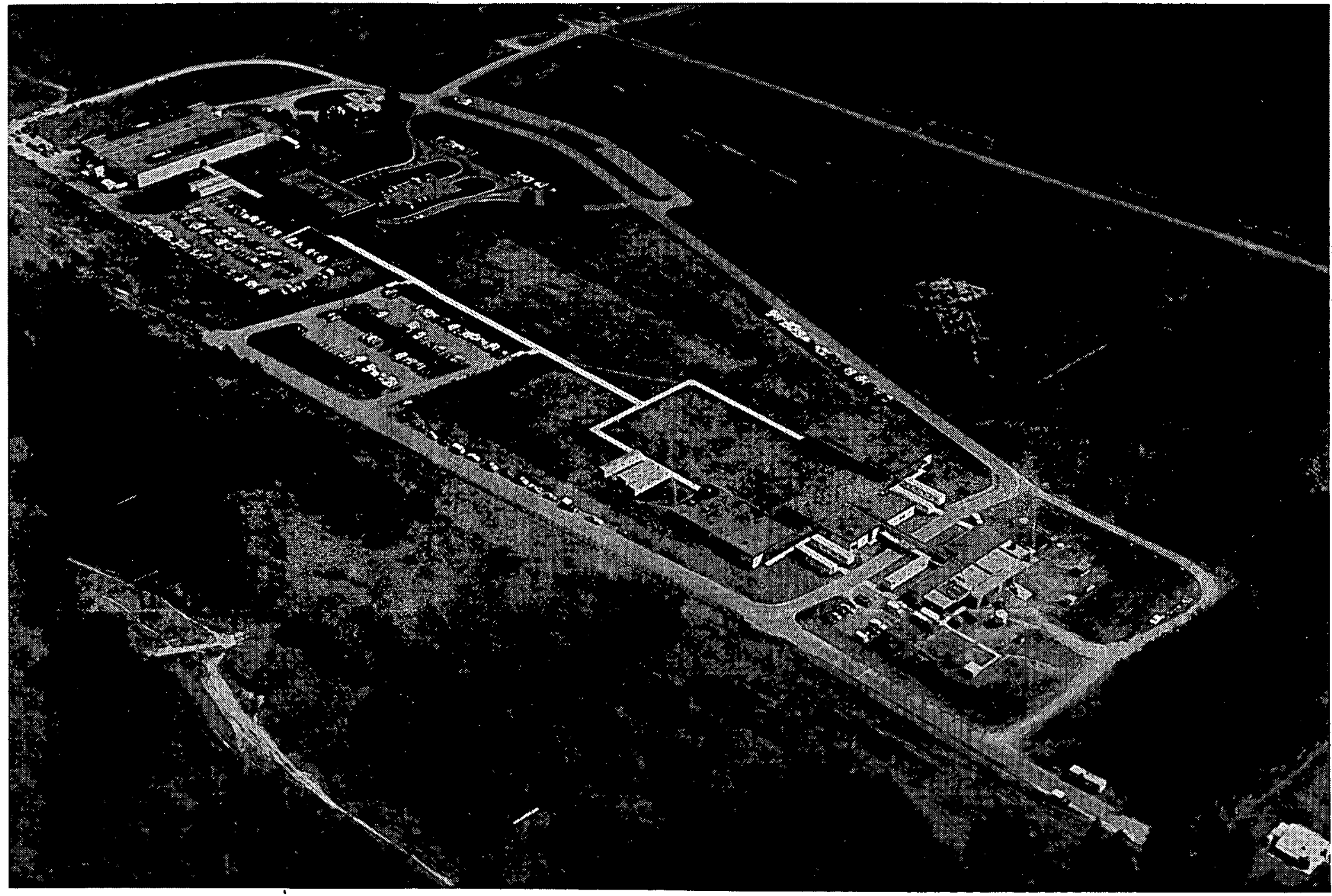




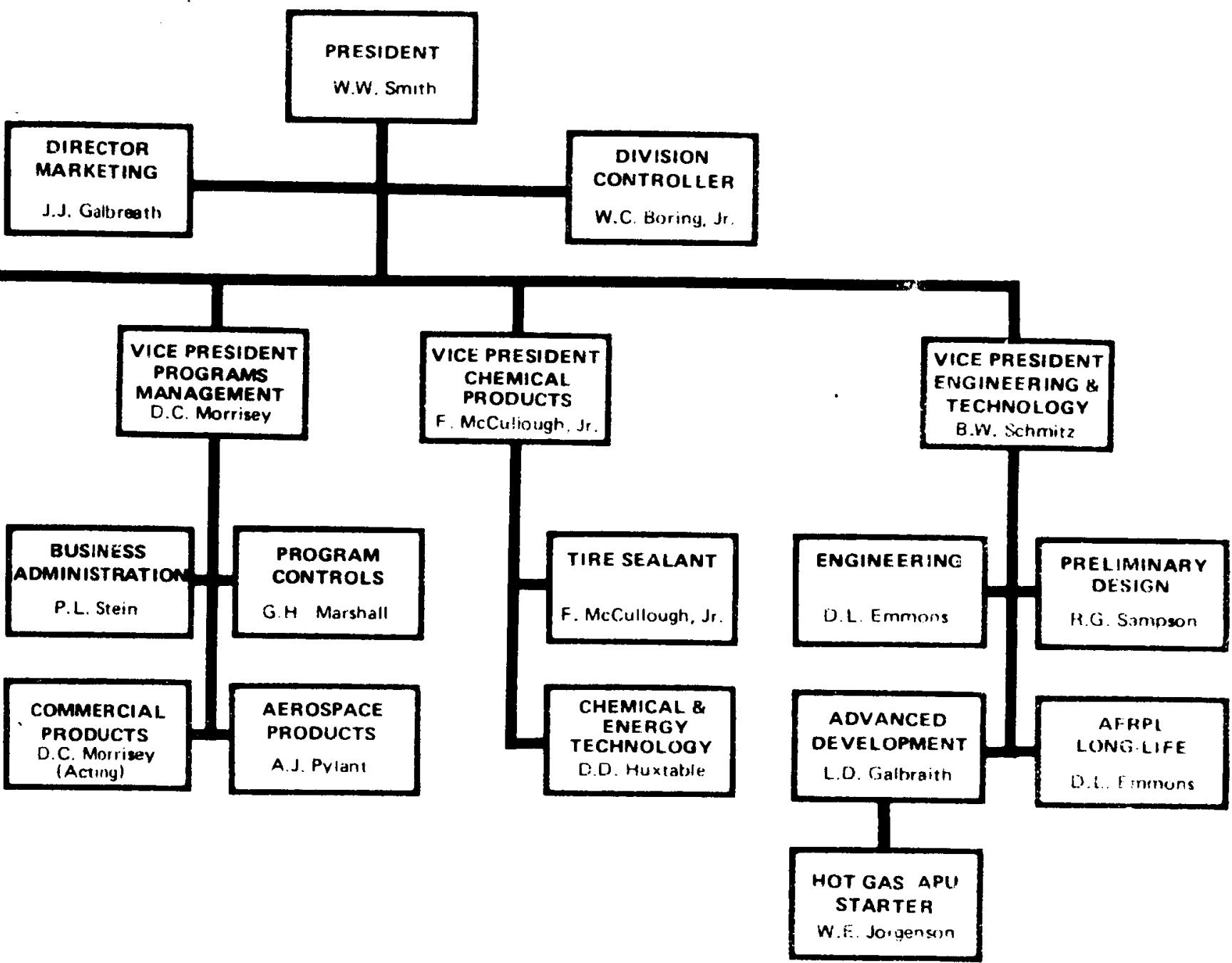

FACILITIES MAINTENANCE

D.H. Oliver 


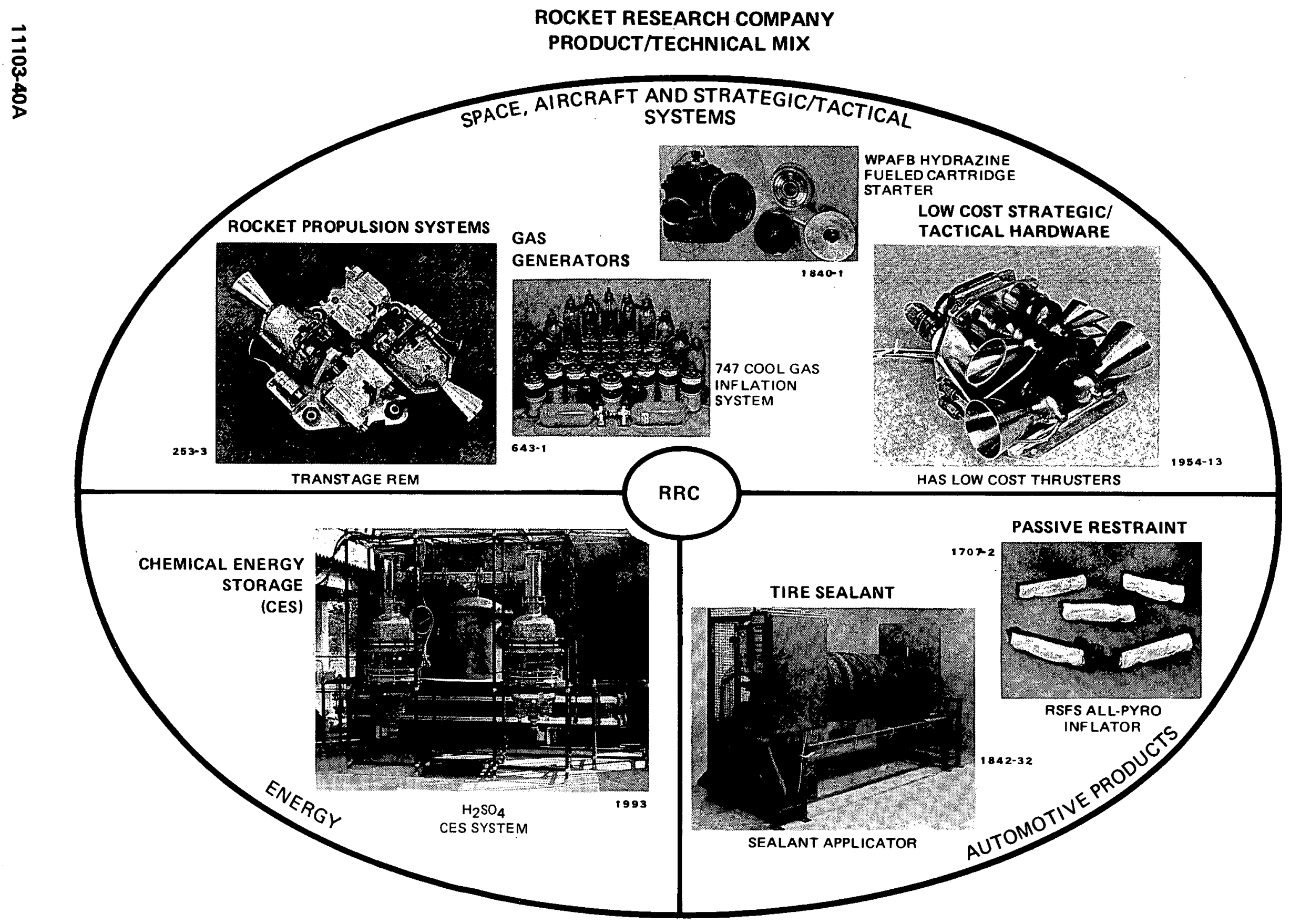


SPACE SHUTTLE APU GAS GENERATOR

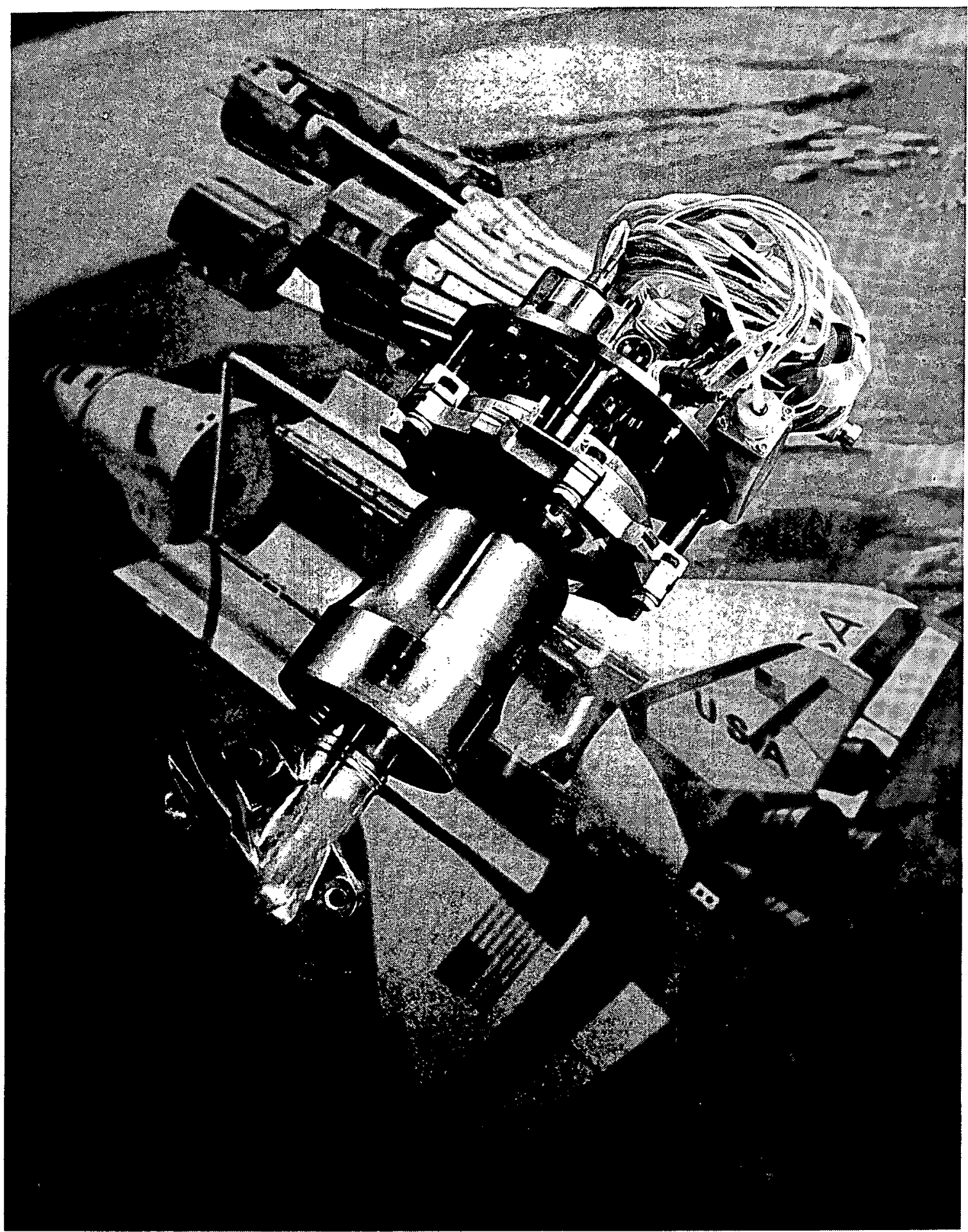




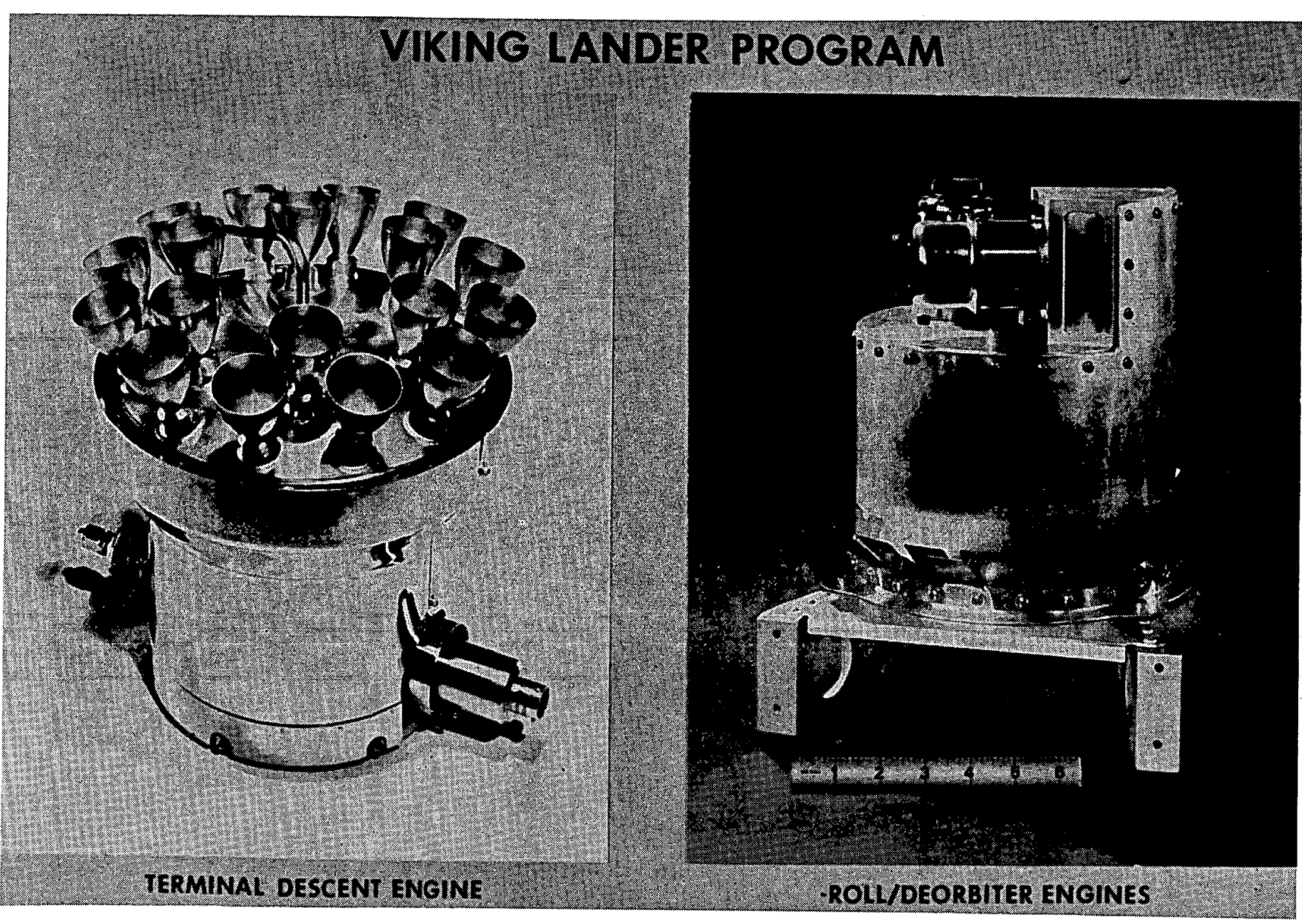


VIEW OF RRC TERMINAL DESCENT ENGINE FROM MARS

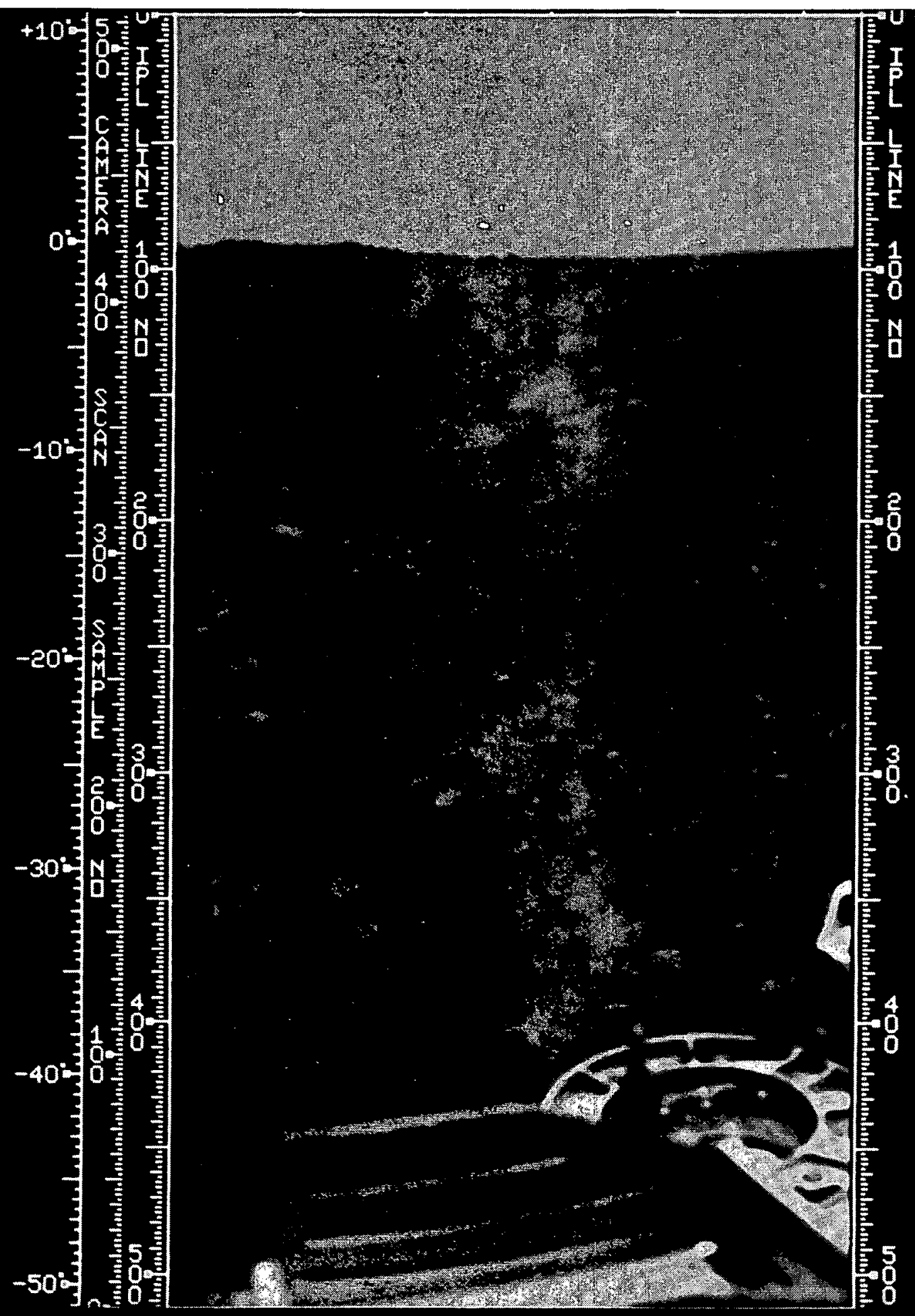




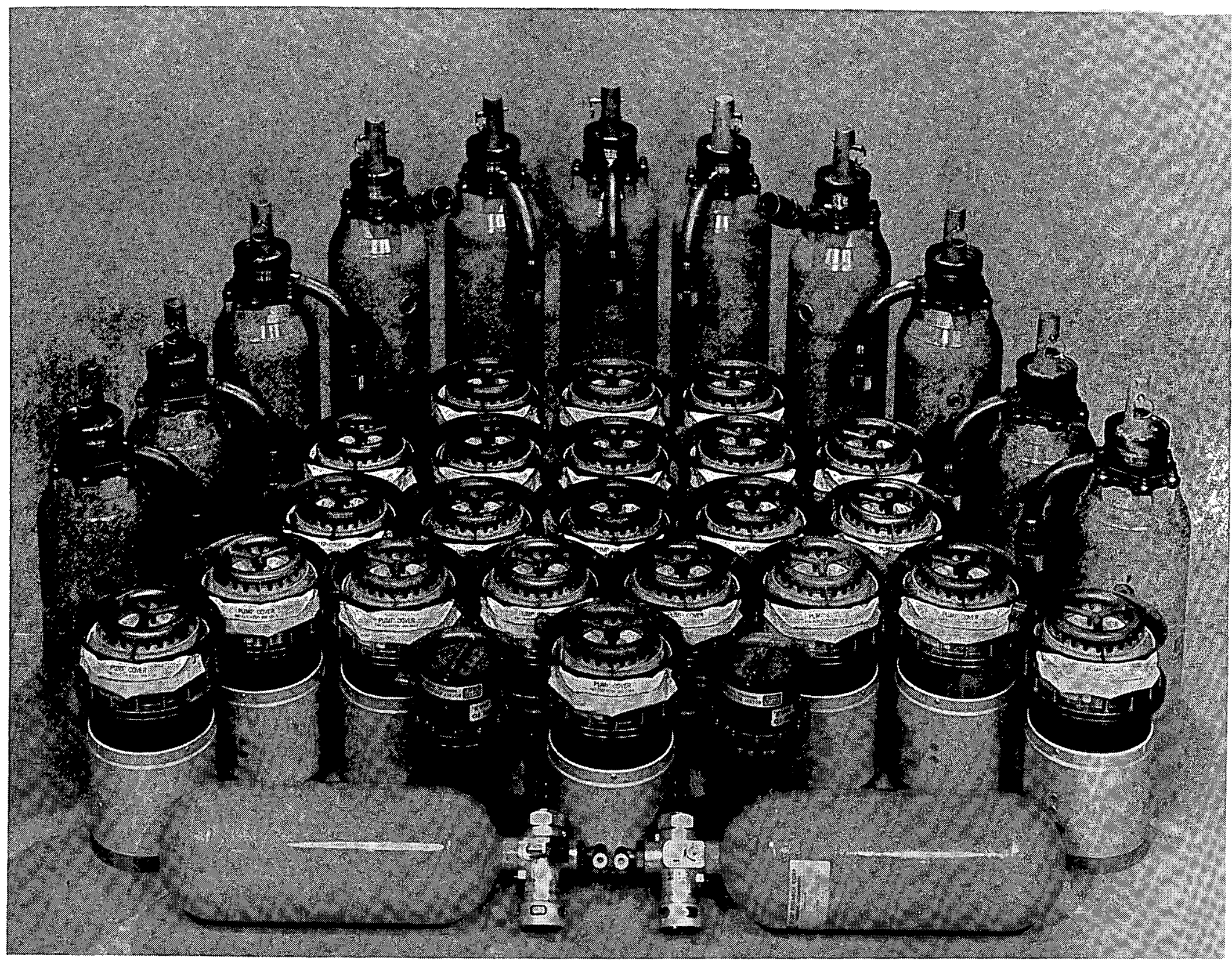


¿

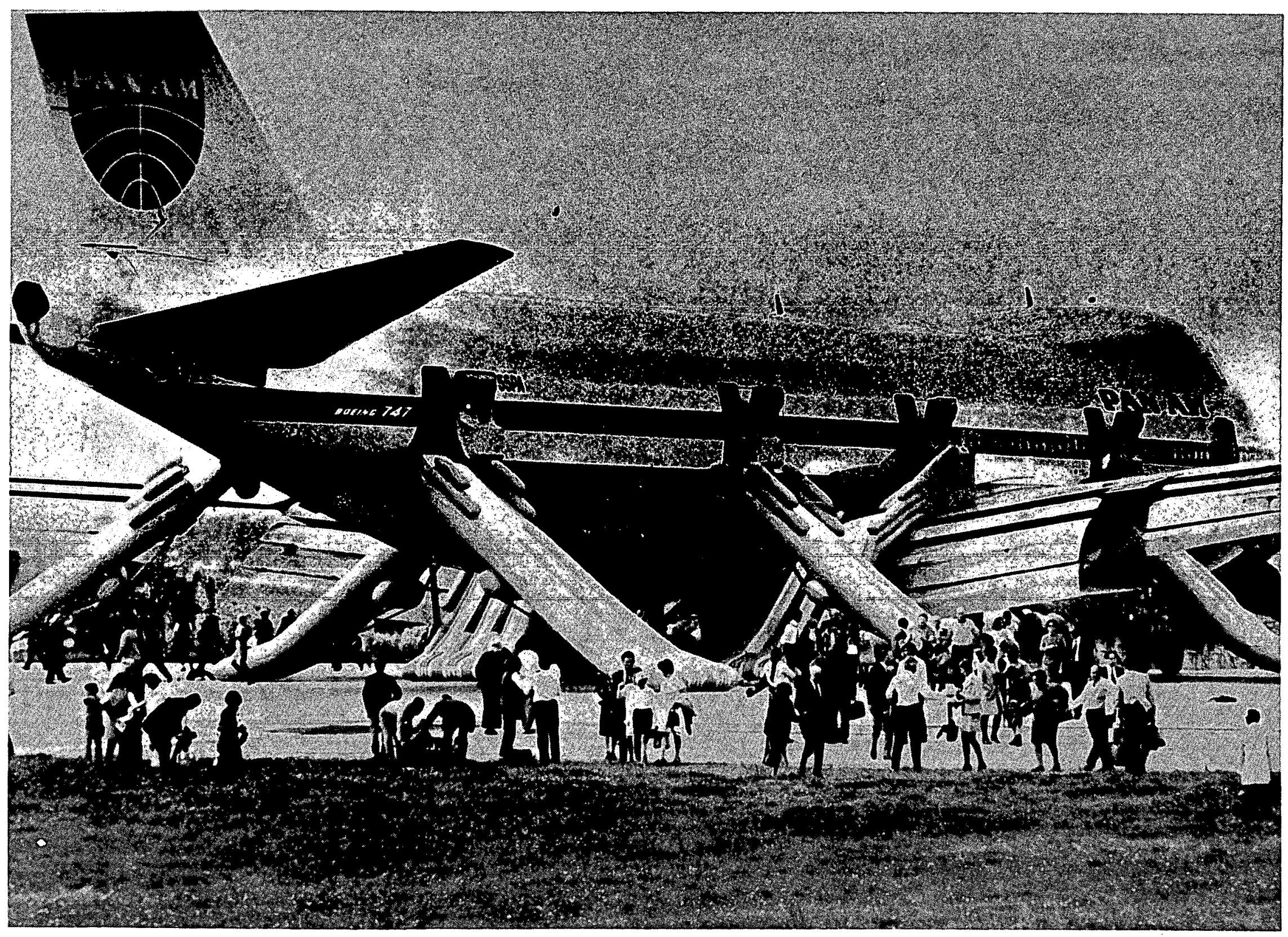


ROCKCOR, INC.

ENERGY TECHNOLOGY

\begin{tabular}{|c|c|c|c|c|c|c|c|}
\hline & \multicolumn{4}{|c|}{ CURAENT RESOURCES } & \multicolumn{3}{|c|}{ FUTURE RESOURCES } \\
\hline & OHL \&AS & COAL & FISSION & $\begin{array}{c}\text { POWER } \\
\text { TRANSMISSION/ } \\
\text { CONVIUSIISN }\end{array}$ & SOI.AH & GtOTHF HMAI & H1Main \\
\hline $\begin{array}{l}\text { ROCKET } \\
\text { RESEARCH } \\
\text { COMPANY }\end{array}$ & $\begin{array}{l}\text { WASTE HEAT } \\
\text { RECOVERY }\end{array}$ & $\begin{array}{l}\text { CATALYST R\&O } \\
\text { FOR METHANATION } \\
\text { WASTE HEAT } \\
\text { RECOVERY }\end{array}$ & $\begin{array}{l}\text { WASTE HEAT } \\
\text { HECOVERY }\end{array}$ & $\begin{array}{l}\text { EXPLUSIVE } \\
\text { SWITCHES O } \\
\text { EXPANSION } \\
\text { ENGINES } \\
\text { WASTE HEAT } \\
\text { HECUVEAY }\end{array}$ & $\begin{array}{l}\text { ENEAGY STOAAGE } \\
\text { CHEMICAL } \\
\text { PHASE CHANGE } \\
\text { FUEI. CELI. }\end{array}$ & $\begin{array}{l}\text { HIGH TEMP } \\
\text { EXPLOSIVE } \\
\text { FOR STIMU. } \\
\text { LATION }\end{array}$ & \\
\hline $\begin{array}{l}\text { PHYSICS } \\
\text { INTERNATIONAL } \\
\text { COMPANY }\end{array}$ & $\begin{array}{l}\text { DYNAFRAC } \\
\text { PROCESS } \\
\text { SONIC. JET } \\
\text { IGNITION }\end{array}$ & $\begin{array}{l}\text { COAL FHAC - RECOVERY } \\
\text { OF METHANE } \\
\text { EB PROCESSING }\end{array}$ & $\begin{array}{l}\text { ELECTRIC BREEDER } \\
\text { FOR FUEL SUPPLY } \\
\text { PICSES ANALY } \\
\text { SIS OF NUCLEAR } \\
\text { REACTOR SAFETY }\end{array}$ & $\begin{array}{l}\text { HIGH } \\
\text { SPEED } \\
\text { SWITCHING }\end{array}$ & & & $\begin{array}{l}\text { ADVANCED CUNCIFIS } \\
\text { INCLUDING E BEAM } \\
\text { HEATED SOLENOSII ANU } \\
\text { PELLET COMHHE SSIIIN }\end{array}$ \\
\hline $\begin{array}{l}\text { PETHOLEUM } \\
\text { IECHNOLOGY } \\
\text { CORPOHATION }\end{array}$ & $\begin{array}{l}\text { CHEMICAL EX } \\
\text { PLOSIVE } \\
\text { STIMULATION }\end{array}$ & & $\begin{array}{l}\text { IN SIIU LEACH } \\
\text { MINING OF } \\
\text { URANIUM ORES }\end{array}$ & & & $\begin{array}{l}\text { EXPLOSIVE } \\
\text { STIMULAIION } \\
\text { PHOCESS }\end{array}$ & \\
\hline $\begin{array}{l}\text { LARSE } \\
\text { CORPOHATION }\end{array}$ & $\begin{array}{l}\text { PIPELINE } \\
\text { MONITORING } \\
\text { AND CONTROL } \\
\text { SYSTEMS }\end{array}$ & & & $\begin{array}{l}\text { STATUS } \\
\text { MONITORING } \\
\text { SYSTEMS }\end{array}$ & & & \\
\hline
\end{tabular}

Ophoposals

unger cunthact 


\section{LARSE CORPORATION PRODUCTS}

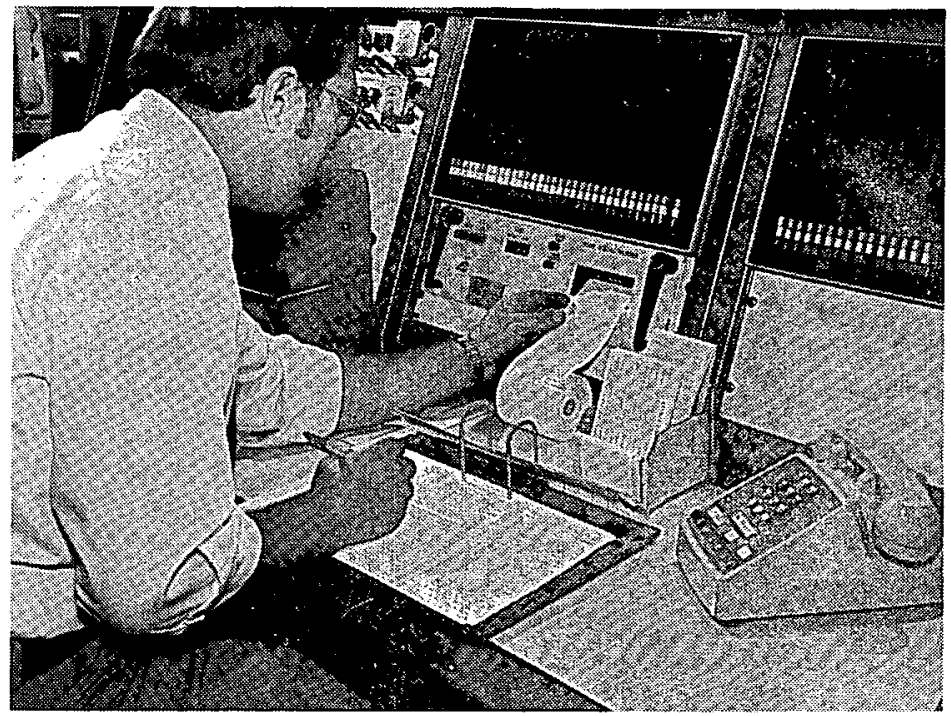

LARSE 3002

MULTIPOINT ALARM SYSTEM

MASTER TERMINAL UNITS
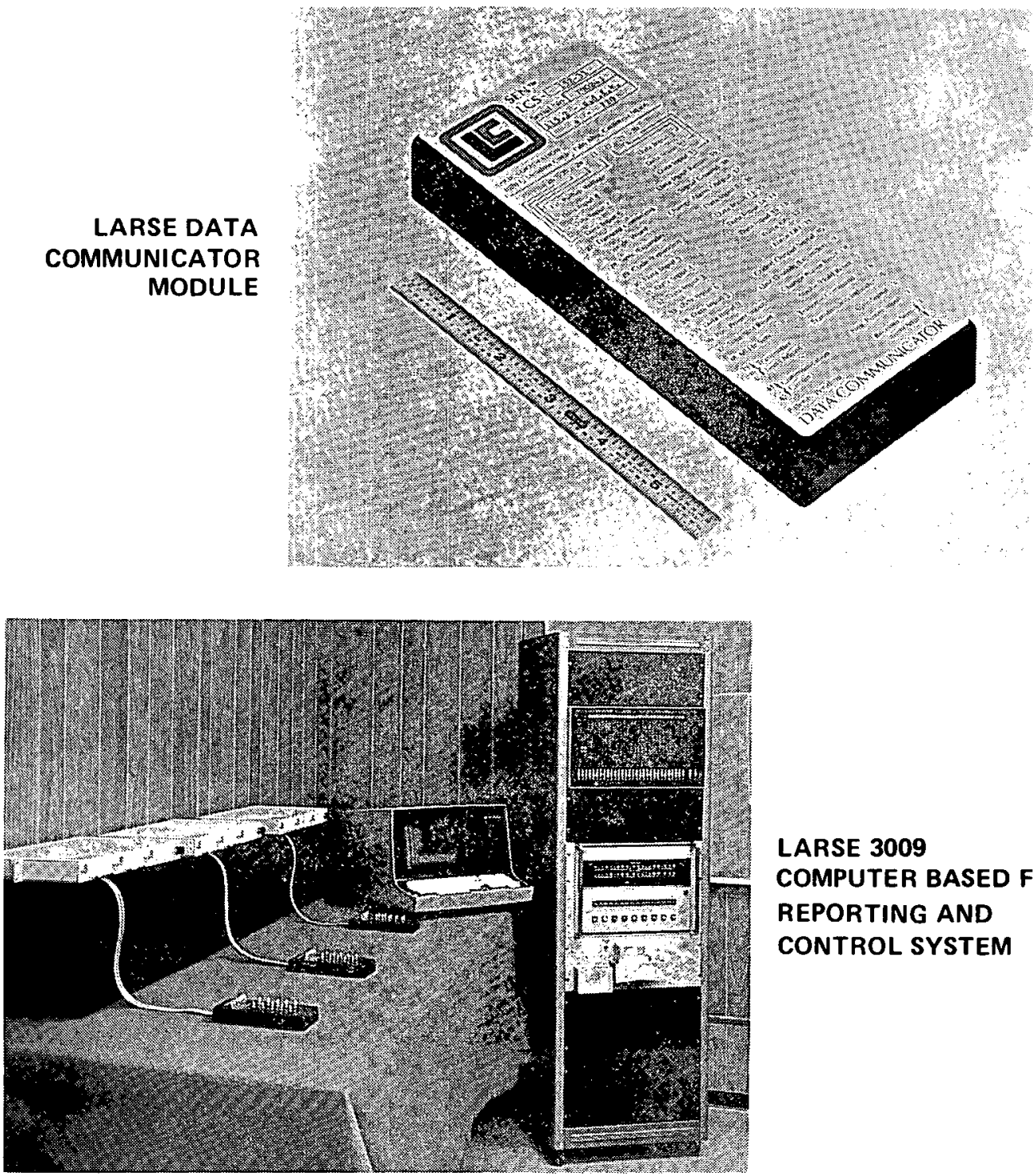

LARSE 3009

COMPUTER BASED FAULT

REPORTING AND

CONTROL SYSTEM 
PETROLEUM TECHNOLOGY CORPORATION

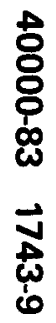

ASTRO FLOW II CHEMICAL EXPLOSIVE FRACTURING EQUIPMENT

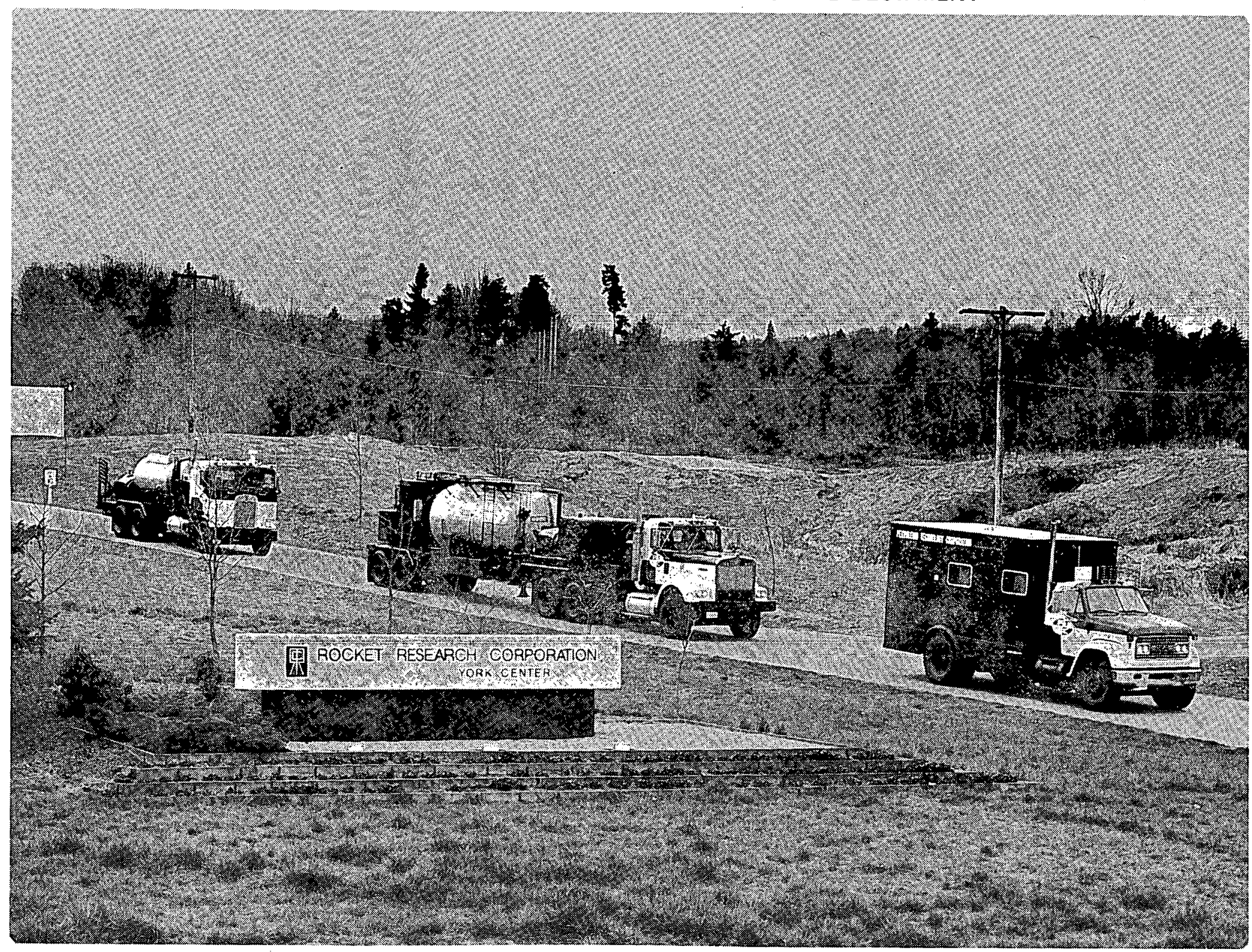


PTC REMOTE CONTROL WELL HEAD “CHRISTMAS TREE"

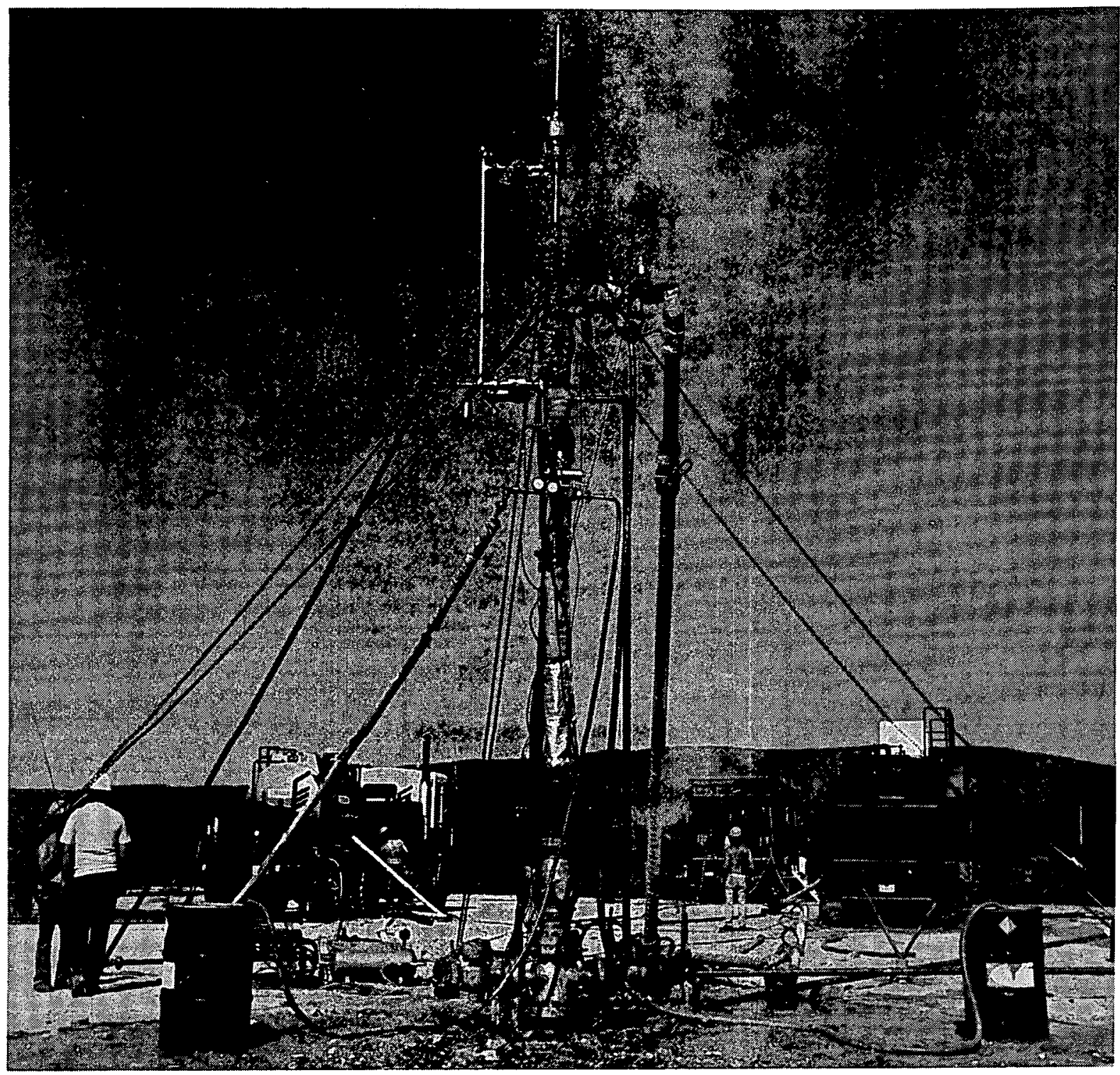




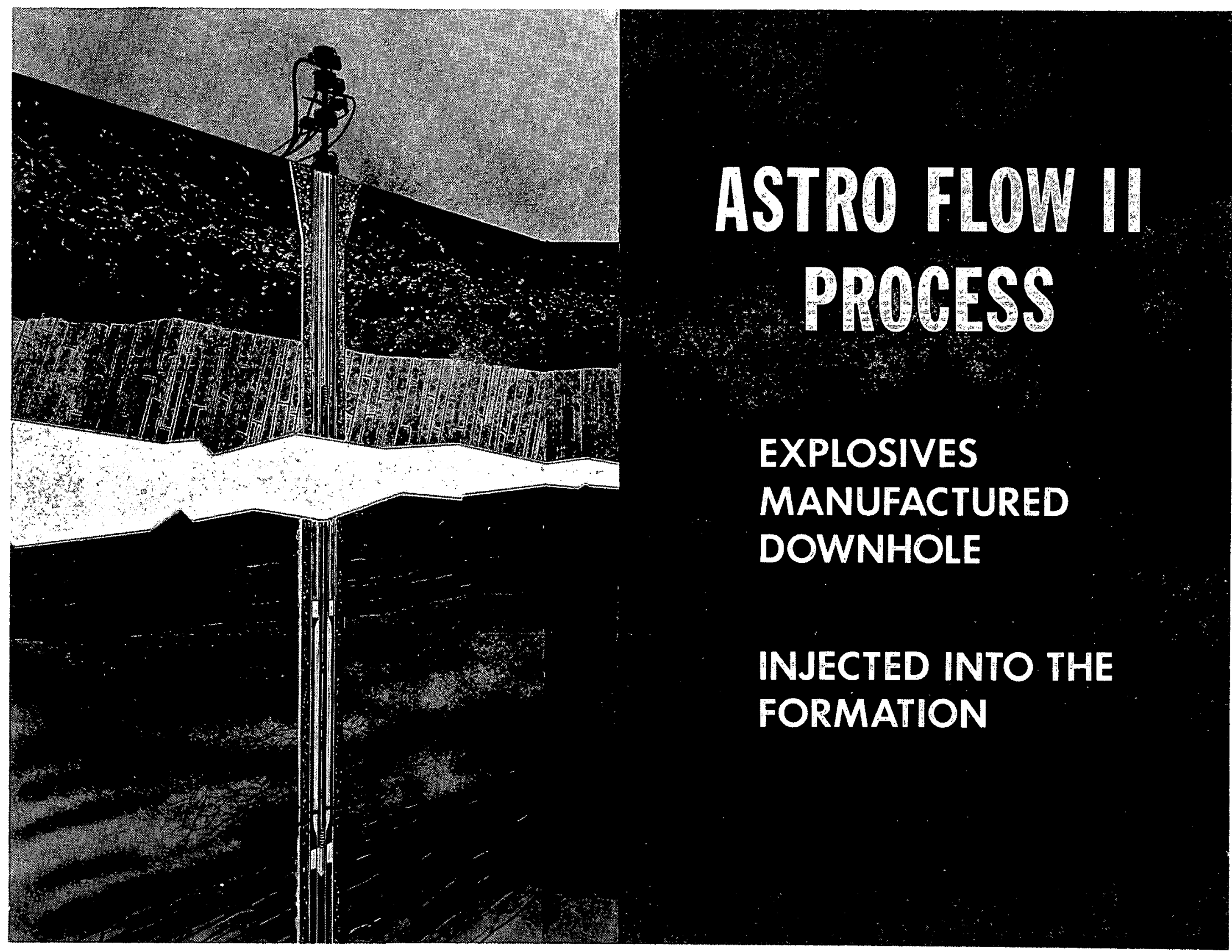


PHYSICS INTERNATIONAL PRODUCTS

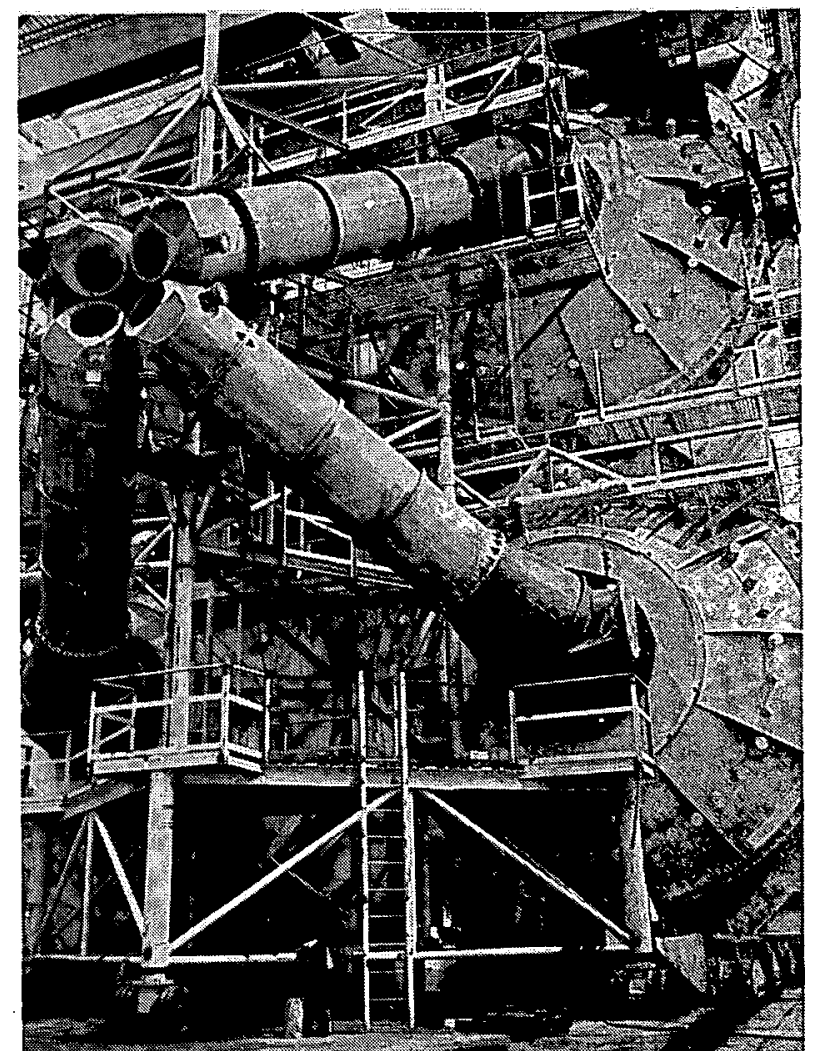

AURORA FLASH X-RAY MACHINE INSTANTANEOUS POWER LEVEL OF 25 MILLION-MILLION WATTS

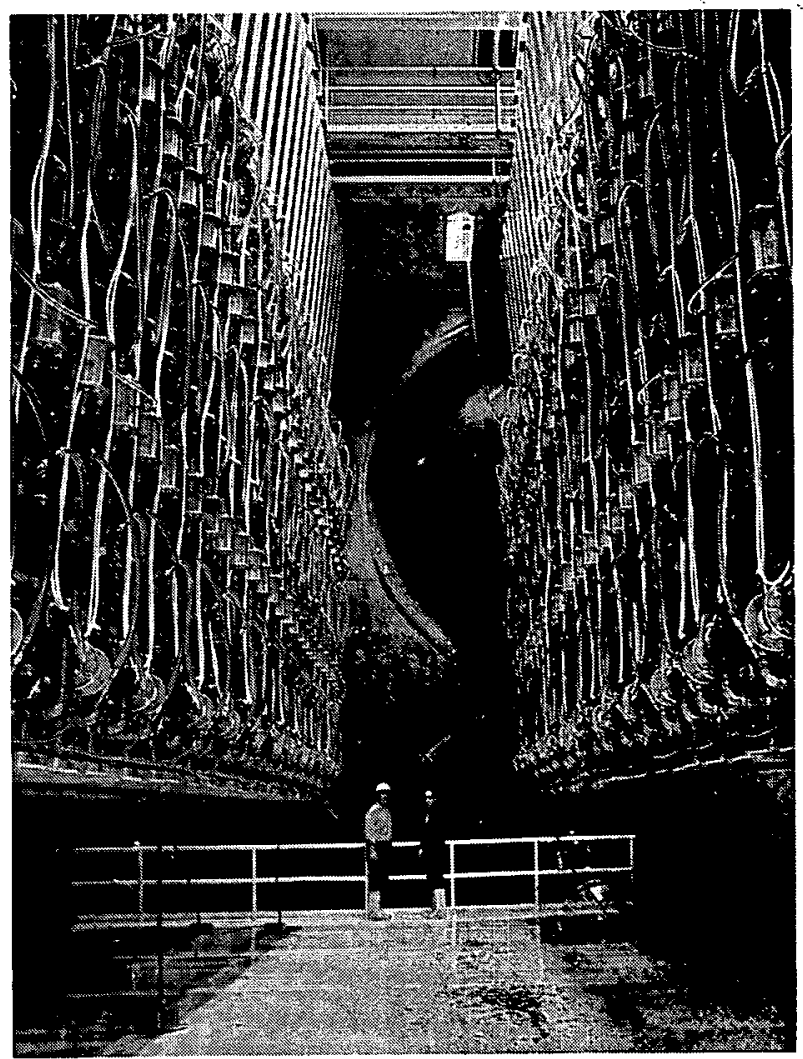

INTERIOR OF AURORA X-RAY MACHINE

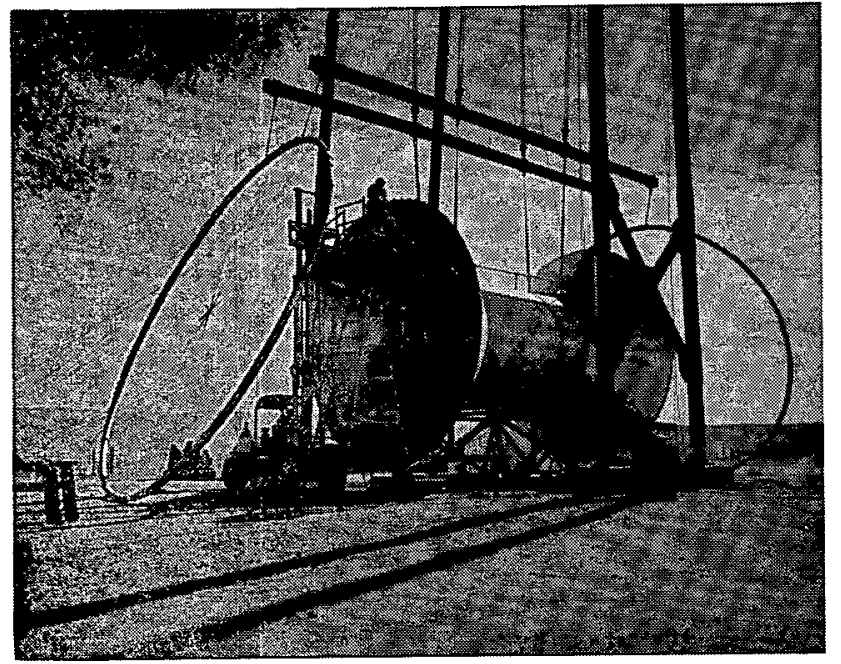

TEMPS GENERATOR PRODUCES ELECTROMAGNETIC PULSE TO SIMULATE NUCLEAR ENVIRONMENT FOR ELECTRONIC COMPONENT TESTING

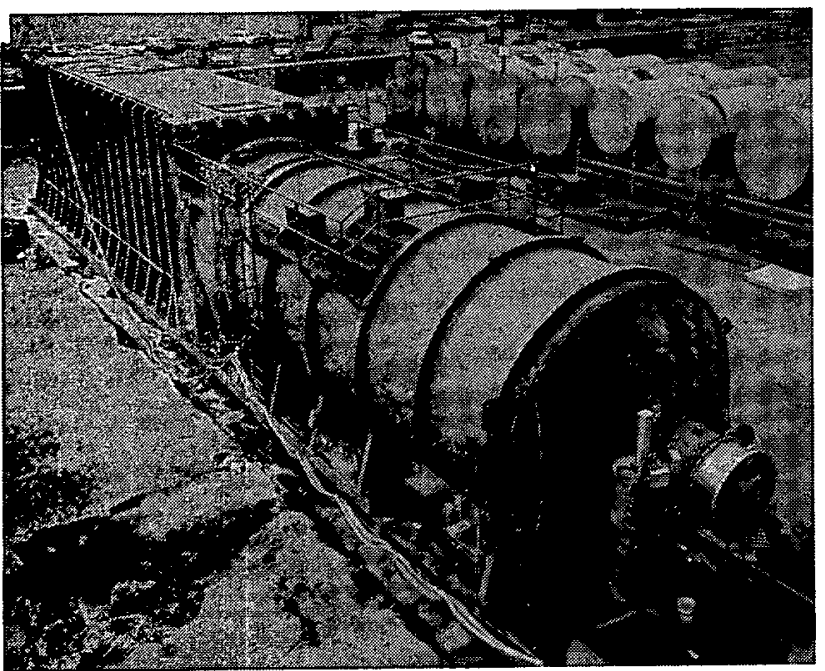

PULSERAD 1480 BUILT FOR FRENCH GOVERNMENT. POWER OUTPUT EXCEEDS 1 TRILLION WATTS.

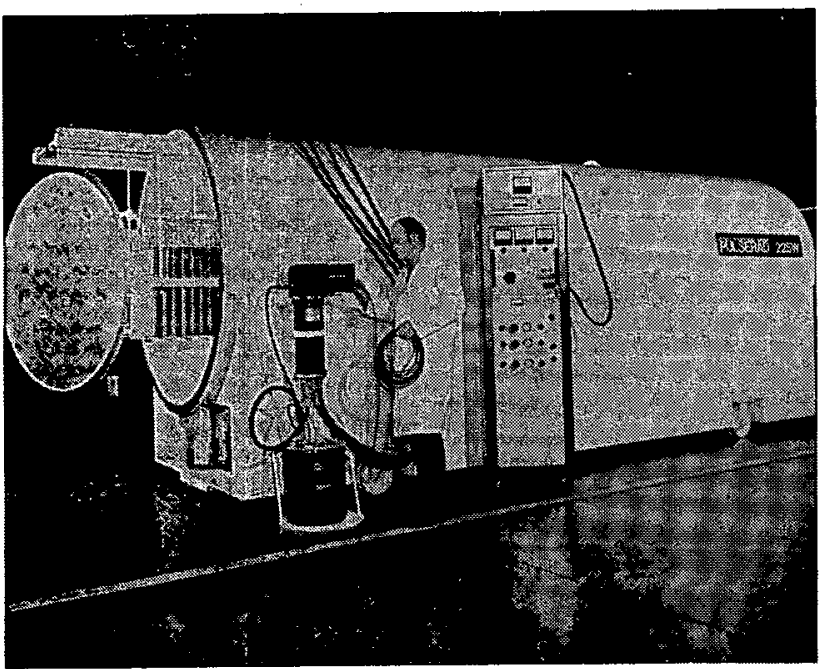

PULSERAD 225-W ELECTRON BEAM ACCELERATOR. OUTPUT OF 1 MILLION ELECTRON VOLTS AT 200,000 AMPERES. 
A HIGH-TEMPERATURE EXPLOSIVE FOR EXPLORATION AND DEVELOPMENT OF GEOTHERMAL ENERGY SOURCES

\section{OBJECTIVE}

- TWO-COMPONENT LIQUID EXPLOSIVE

- NONDETONABLE COMPONENTS

- CAPABLE OF WITHSTANDING TEMPERATURE ENVIRONMENT IN GEOTHERMAL WELLS

- STABLE fOR AT LEASE 24 HOURS AT $477^{\circ} \mathrm{K}\left(400^{\circ} \mathrm{F}\right)$ OR SHORTER PERIODS AT UP TO $561^{\circ} \mathrm{K}\left(550^{\circ} \mathrm{F}\right)$ 
APPLICATIONS OF HIGH-TEMPERATURE EXPLOSIVE

VAPOR-DOMINATED RESERVOIRS

- INCREASE FLOW RATE IN PRODUCING WELLS

- Stimulate maRginal Wells

- SAVE DRILLING TIME BY STIMULATING SHALLOW WELLS

LIQUID-DOMINATED RESERVOIRS

- INCREASE PERMEABILITY IN PRODUCING WELLS

- REMOVE SCALE DEPOSITS IN REINJECTION WELLS

DRY HOT ROCK BEDS

- CONNECTING INJECTION AND PRODUCTION WELLS

- "RUBblizING" OF ROCK to INCREASE SURFACE AREA AND ACCELERATE RATE OF HEAT TRANSFER 
PUBLISHED RESULTS OF EXPLOSIVE STIMULATION TREATMENTS

\begin{tabular}{|c|c|c|c|c|}
\hline $\begin{array}{c}\text { Area } \\
\text { or } \\
\text { Company }\end{array}$ & $\begin{array}{c}\text { No. } \\
\text { of } \\
\text { Wells }\end{array}$ & $\begin{array}{c}\text { Average } \\
\text { Production } \\
\text { Before Slıot }\end{array}$ & $\begin{array}{c}\text { Average } \\
\text { Production } \\
\text { After Sliot }\end{array}$ & $\begin{array}{c}\text { Improvement } \\
\text { Ratio } \\
\text { Avg \& Max }\end{array}$ \\
\hline Kentucky & 6,370 & $53 \mathrm{MCF} / \mathrm{D}$ & $253 \mathrm{MCF} / \mathrm{D})$ & $4.8 \cdot 250$ \\
\hline Western Co. & 8 & $21 \mathrm{BBL} / \mathrm{D}$ & $80.5 \mathrm{BBL} / \mathrm{I})$ & $3.8 \cdots 12$ \\
\hline Dowell & 4 & \multicolumn{2}{|c|}{ Oil Wells } & $13.6-25$ \\
\hline & 6 & \multicolumn{2}{|c|}{ Oil Wells } & $14.4 \ldots \quad 23$ \\
\hline \multirow[t]{2}{*}{ Talley } & 3 & $260 \mathrm{MCF} / \mathrm{D}$ & $998 \mathrm{MCF} / \mathrm{D}$ & 3.8 \\
\hline & 4 & $11.6 \mathrm{BBL} / \mathrm{D}$ & $30.3 \mathrm{BBL} / \mathrm{I})$ & $2.6-14$ \\
\hline
\end{tabular}


USBM/ERDA CHEMICAL EXPLOSIVE FRACTURING OF GAS WELLS

SUMMARY OF RESULTS

\begin{tabular}{|c|c|c|c|c|}
\hline $\begin{array}{c}\text { Well } \\
\text { Designation }\end{array}$ & $\begin{array}{l}\text { Amo } \\
\text { Expl } \\
\text { Inje }\end{array}$ & $\begin{array}{l}\text { it of } \\
\text { sive } \\
\text { ed }\end{array}$ & Well History & Results \\
\hline Hampshire No. 10 & 9,149 & 20,170 & Drilled 1964, acidized once, & $\begin{array}{l}\text { No change. Hole diameter increised } \\
\text { from } 6 \text { inches }(15 \mathrm{~cm}) \text { to } 9-10 \\
\text { inches }(22 \text { to } 25 \mathrm{~cm})\end{array}$ \\
\hline Hampshire No. 18 & 10,333 & 22,780 & $\begin{array}{l}\text { Drilled } 1968, \text { acidized twice, } \\
\text { no improvement as result of } \\
\text { previous treitments }\end{array}$ & Inflow improved by factor of 1.66 \\
\hline Columbia No. 20117-T & 13,753 & 30,320 & $\begin{array}{l}\text { Completed } 1975, \text { no previous } \\
\text { stimulation attempts }\end{array}$ & $\begin{array}{l}\text { Productivity increased. Well could } \\
\text { not be cleaned out }\end{array}$ \\
\hline
\end{tabular}


EXPLOSIVES SELECTION FOR GEOTHERMAL WELL STIMULATION

\begin{tabular}{|c|c|c|}
\hline $\begin{array}{l}\text { PHYSICAL } \\
\text { STATE }\end{array}$ & ADVANTAGES & DISADVANTAGES \\
\hline SOLID & $\begin{array}{l}\text { HIGH ENERGY DENSITY. EXISTING } \\
\text { HIGH-TEMPERATURE RESISTANT } \\
\text { SOLID EXPLOSIVES. }\end{array}$ & $\begin{array}{l}\text { EXPLOSIVE HAZARD ABOVE GROUND. } \\
\text { EXPENSIVE. HOLE CANNOT BE ENTIRELY } \\
\text { FILLED WITH SOLIDS. }\end{array}$ \\
\hline LIQUID & $\begin{array}{l}\text { HIGH ENERGY DENSITY. } \\
\text { CLOSE COUPLING POSSIBLE. }\end{array}$ & $\begin{array}{l}\text { NOT ALL LIOUIDS ARE READILY MISCIBLE. } \\
\text { LIQUIDS ARE GENERALLY THERMALLY } \\
\text { LESS STABLE THAN SOLIDS. }\end{array}$ \\
\hline GASEOUS & $\begin{array}{l}\text { READILY MIXED. } \\
\text { CLOSE COUPLING POSSIBLE }\end{array}$ & $\begin{array}{l}\text { LOW ENERGY DENSITY. } \\
\text { ONLY FEW COMBINATIONS KNOWN. }\end{array}$ \\
\hline
\end{tabular}


PROPERTIES OF EXPLOSIVES UNDER CONSIIERATION FOR GEOTHERMAL APPLICATION

\begin{tabular}{|c|c|c|c|c|c|c|c|}
\hline Explosive & $\begin{array}{l}\text { Density } \\
\mathrm{gm} / \mathrm{cm}^{3}\end{array}$ & $\begin{array}{l}\text { Melting } \\
\text { Point } \\
\text { oK }\end{array}$ & $\begin{array}{c}\text { Current } \\
\text { Thicrmal } \\
\text { Application } \\
\text { Range } \\
\text { oK }\end{array}$ & $\begin{array}{l}\text { Detonation } \\
\text { Velocity } \\
\text { m/sec }\end{array}$ & $\begin{array}{l}\text { Detonation } \\
\text { Pressure } \\
\text { K bar }\end{array}$ & $\begin{array}{l}\text { Impact(1) } \\
\text { Sensitivity } \\
\text { cm (solids) } \\
\text { Joules/sec } \\
\text { (licpuids) }\end{array}$ & Comments \\
\hline \multicolumn{8}{|l|}{ Solids } \\
\hline Z-TACOT & 1.85 & 683 & 561589 & 7.200 & $1 \times 0$ & & $\begin{array}{l}\text { Commercially available. Solc } \\
\text { source supply until patent } \\
\text { expires }\end{array}$ \\
\hline HNS & 1.74 & 591 & $533-561$ & 7.100 & 230 & 39 & Commercially availahlc \\
\hline PYX & 1.77 & - & $561-589$ & 7,800 & 270 & 6.3 & $\begin{array}{l}\text { Developed by LASI. curremly } \\
\text { no commercial soarce }\end{array}$ \\
\hline PATO & 1.94 & 593 & $505-533$ & . & 307 & 320 & $\begin{array}{l}\text { Developed hy LASL . currenly } \\
\text { no commercial source }\end{array}$ \\
\hline DATB & 1.84 & 562 & $505 \ldots 533$ & 7.700 & 282 & 320 & $\begin{array}{l}\text { Used in military service. } \\
\text { commercially available }\end{array}$ \\
\hline TATB & 1.94 & (2) & $533-561$ & 7.970 & 313 & 320 & $\begin{array}{l}\text { May be commercially atvailable } \\
\text { in near future. ERIDA-contrulled } \\
\text { supply }\end{array}$ \\
\hline
\end{tabular}

Notes:

(1) Type 12 impact tool for solid explosives

(2) Does not melt prior to decomposition 


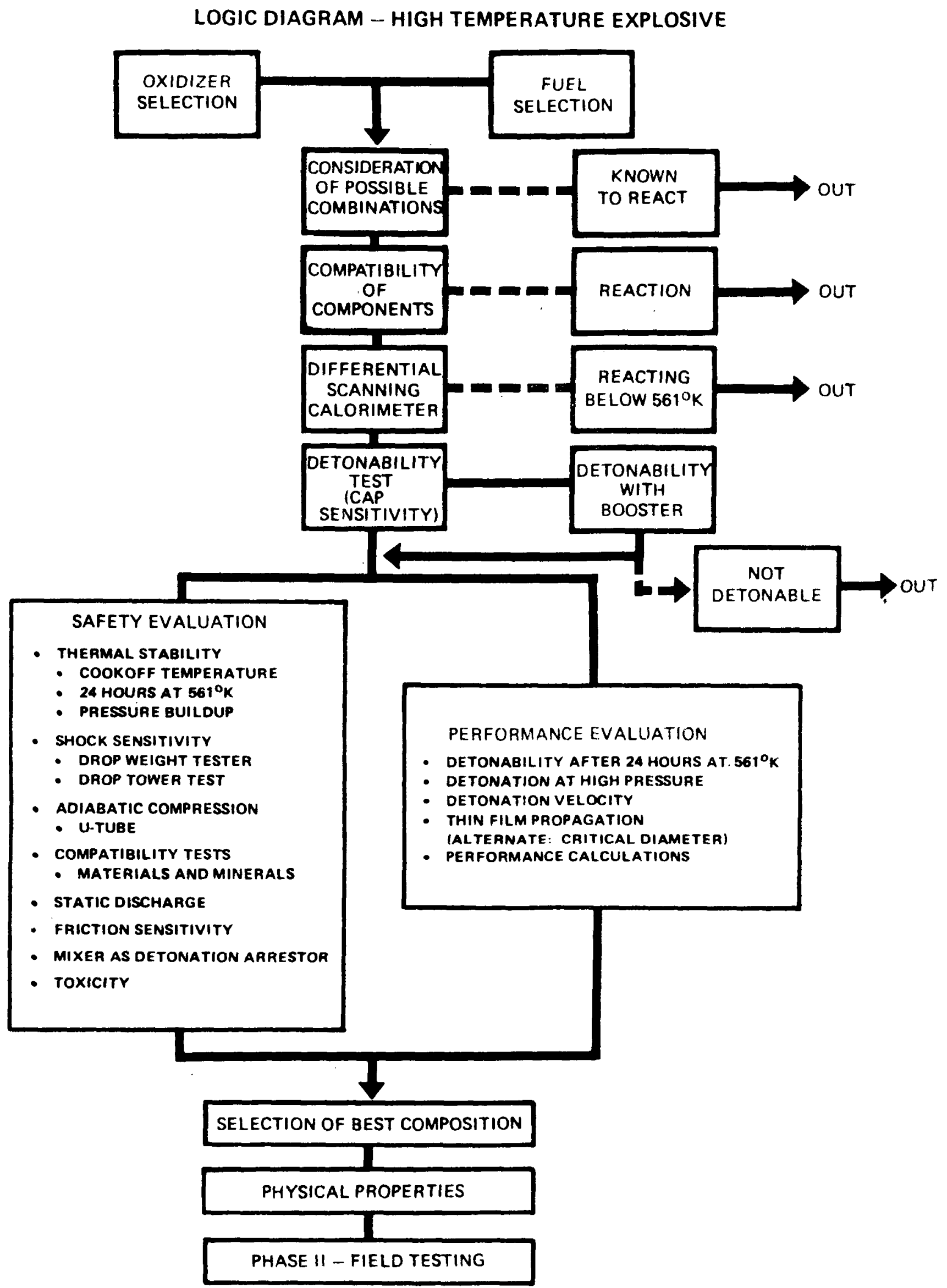


PHYSICAL PROPERTIES OF INORGANIC OXIIIZERS

\begin{tabular}{|c|c|c|c|c|c|c|c|c|c|}
\hline \multirow[t]{2}{*}{ Compound } & \multirow[t]{2}{*}{ Formula } & \multirow[t]{2}{*}{$\begin{array}{c}\text { Molecullar } \\
\text { Weighı }\end{array}$} & \multirow{2}{*}{$\begin{array}{l}\text { Melling } \\
\text { Point } \\
\text { "K }\end{array}$} & \multirow{2}{*}{$\begin{array}{l}\text { Timp. for } \\
\text { Density } \\
\text { OK }\end{array}$} & \multirow[t]{2}{*}{$\begin{array}{l}\text { Density } \\
\text { g/en.3 }\end{array}$} & \multirow{2}{*}{$\begin{array}{l}\text { Uscful } \\
\text { Oxygen } \\
\% \text { by Wi. }\end{array}$} & \multirow{2}{*}{$\begin{array}{l}\text { Usefful } \\
\text { Oxygen } \\
\text { g/cm }\end{array}$} & \multicolumn{2}{|c|}{$\begin{array}{l}\text { Heat of } \\
\text { Formation at } \\
298^{\circ} \mathrm{K}\end{array}$} \\
\hline & & & & & & & & $\mathrm{kcal} / \mathrm{mol}$ & $\mathrm{kJ} / \mathrm{mol}$ \\
\hline Nillic acid $(100 \%)$ & $\mathrm{INO}_{3}$ & 03.0113 & 231 & 298 & $1 . * 1.5027$ & 6.3 .48 & 0.954 & -41.40 & .173 .2 \\
\hline Lillium nitrate & I.iNO 3 & 68.944 & 537 & $\begin{array}{l}293 \\
6.31 .6 \\
882.5\end{array}$ & $\begin{array}{l}S^{*} 2.388 \\
\mathrm{~L} \quad 1.723 \\
1.586\end{array}$ & 58.02 & $\begin{array}{l}1.385 \\
1.000 \\
0.920\end{array}$ & .115 .3 & -482.4 \\
\hline I ilhiimm perchlorate & $\mathrm{Iicl}()_{4}$ & 100.390 & $5(x)$ & 29.3 & $\mathrm{~S} \quad 2.428$ & 60.15 & 1.460 & -90.84 & $-38(1) .3$ \\
\hline Calcium perclilorate & $\left(a\left(\mathrm{ClO}_{4}\right)_{2}\right.$ & 238.98 & 54.3 & 293 & $S \quad 2.6 .51$ & $\$ 3.56$ & 1.42() & -178.11 & .744 .11 \\
\hline Sollium perchlorate & $\mathrm{NaClO})_{4}$ & 122.44 & $75.5(d)$ & 208 & $S \quad 2.5 .36$ & 52.27 & 1.320 & -91.48 & -.382 .7 \\
\hline Calcium nitrate & $\mathrm{Ca}\left(\mathrm{NO}_{3}\right)_{2}$ & 164.09 & 8.34 & 291 & S 2.6.51 & 53.56 & 1.420 & .178 .0 & .744 .11 \\
\hline Sodium millate & $\mathrm{NiNO}_{3}$ & 84.995 & 580 & $\begin{array}{c}293 \\
504.6 \\
\mid(1|| 1.4\end{array}$ & $\begin{array}{ll}S & 2.261 \\
1 . & 1.9(0) \\
1 . & 1.620\end{array}$ & 47.06 & $\begin{array}{l}1.064 \\
0.81) .4 \\
0.76 .2\end{array}$ & -101.5 & -424.7 \\
\hline Nilric acill $(68 \%)$ & $\mathrm{HNO}_{3} \cdot \mathrm{H}_{2} \mathrm{O}$ & & & 293 & I. 1.41 & 4.3 .17 & 0.609 &. & \\
\hline Pulassium perchlorate & $\mathrm{KClO}_{4}$ & 138.55 & 88.3 & 28.3 & S 2.55 & 40.42 & 1.018 & .103 .6 & .433 .0 \\
\hline Potassium nitrate & $\mathrm{KNO}_{3}$ & 101.11 & 607 & $\begin{array}{c}293 \\
6,53.1 \\
1044.7\end{array}$ & $\begin{array}{ll}\mathrm{S} & 2.10 \\
\mathrm{l} . & 1.837 \\
& 1.5 .37\end{array}$ & 39.6 & $\begin{array}{l}0.8 .32 \\
0.727 \\
0.6(04\end{array}$ & .117 .7 & $-4 \% 2.0$ \\
\hline Sexdium nitrite & $\mathrm{NaNO}_{2}$ & $(68.005$ & 544 & 27.3 & $\$ 2.168$ & 34.78 & & -8.5 .4 & -.359 .4 \\
\hline Calcium nitrate tetrahydrate & $\mathrm{Ca}\left(\mathrm{NO}_{3}\right)_{2} \cdot 4 \mathrm{H}_{2} \mathrm{O}$ & 236.15 & 315.8 & 293 & S 1.896 & 33.87 & 0.642 & -509.37 & -21.31 \\
\hline Ammonium perchlorate & $\mathrm{NII}_{4} \mathrm{ClO}_{4}$ & 117.49 & dec. & 29.3 & S 1.95 & 34.04 & 0.664 & -70.74 & .296 .0 \\
\hline Ammonium nitrate & $\mathrm{NH}_{4} \mathrm{NO}_{3}$ & $80.04 ?^{2}$ & 442.8 & 298 & $S \quad 1.725$ & $19.9)$ & 0.345 & -87.9 .3 & .367 .9 \\
\hline
\end{tabular}

- I. $\therefore$ I.icjuid $S=$ Solis 


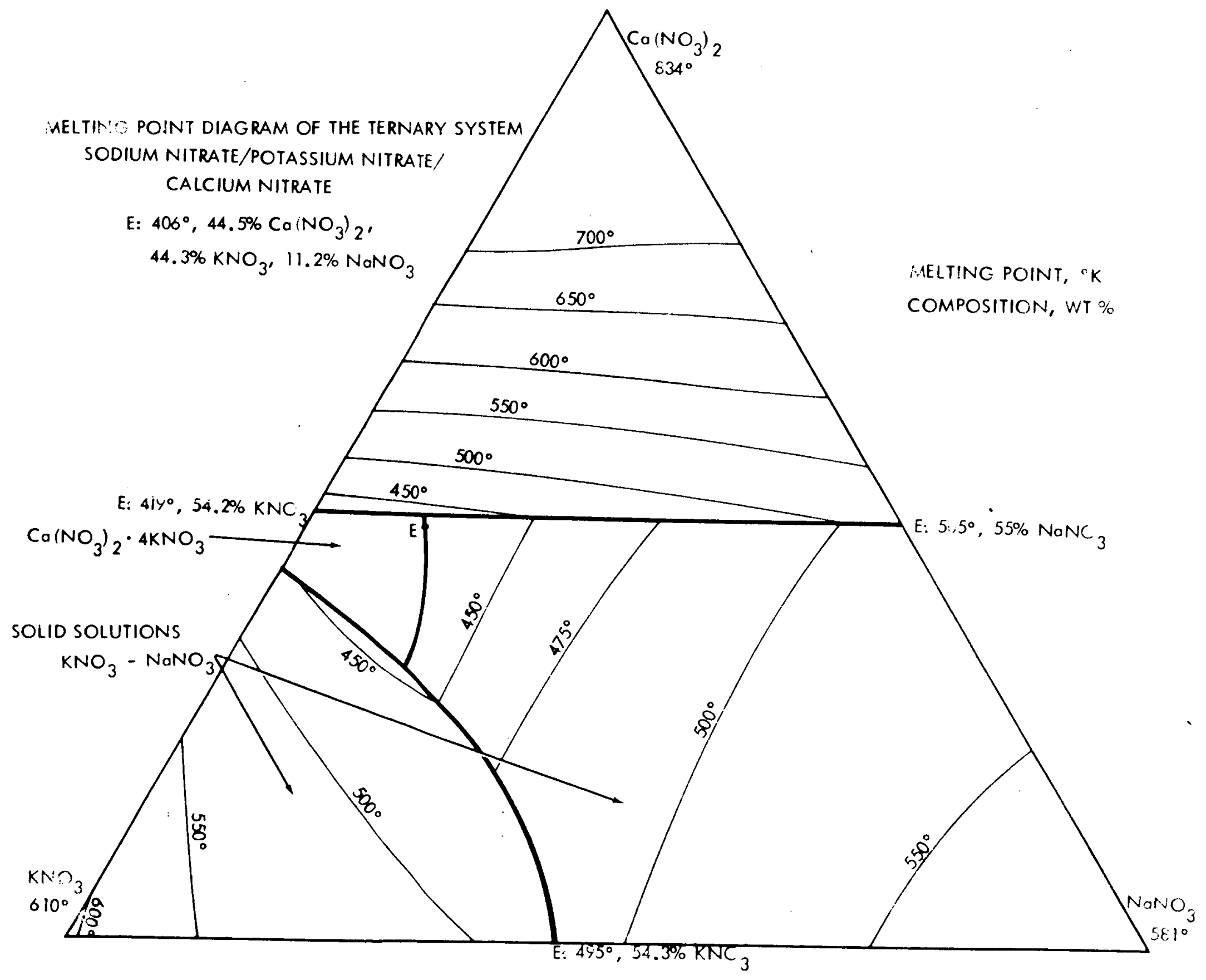




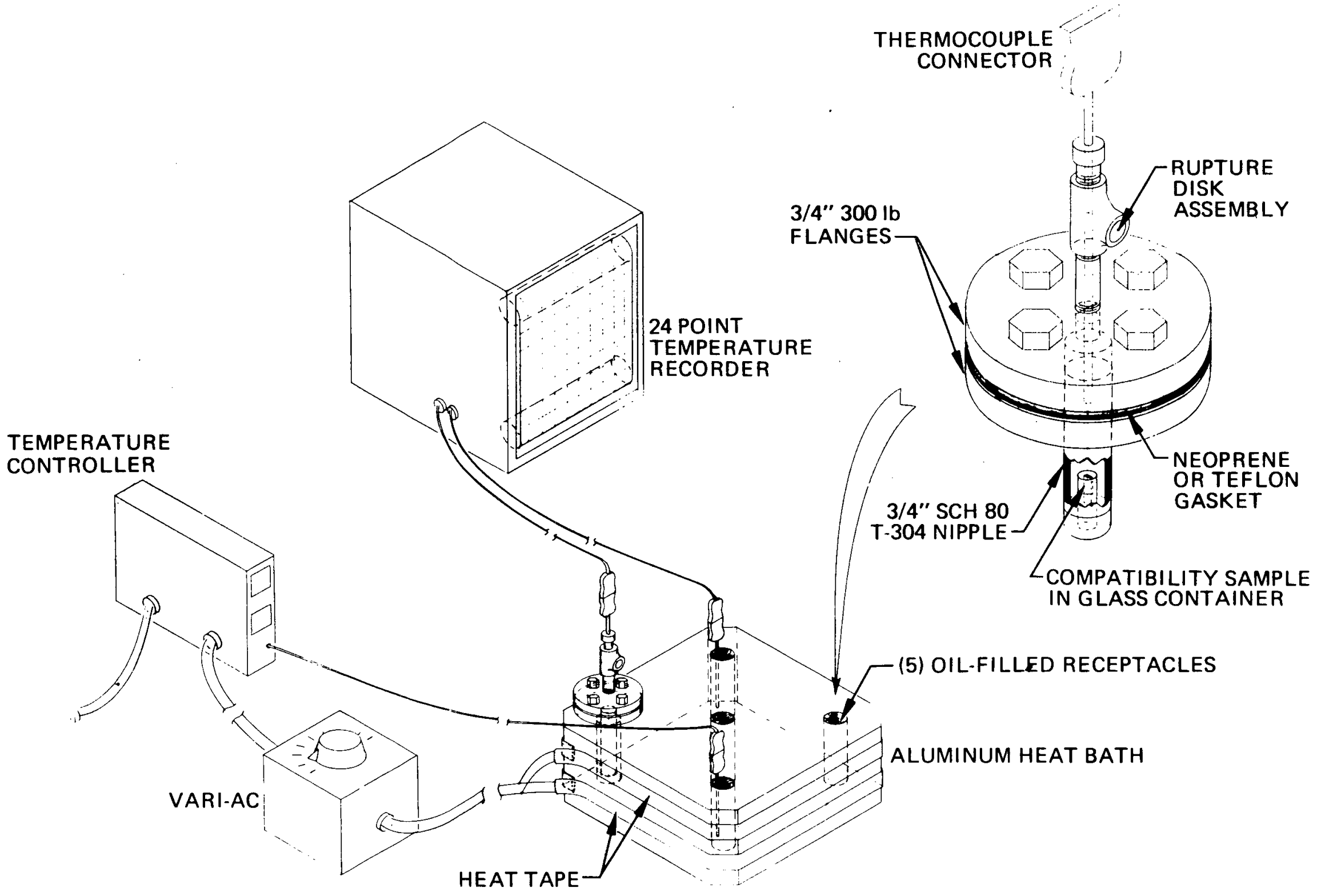


ANALYTICAL RESULTS OF POST-TEST THERIIAL STABILITY SAMPLES - 24 HOURS

\begin{tabular}{|c|c|c|c|}
\hline Sample 1 & $\begin{array}{c}\text { Test } \\
\text { Temp. } \\
1^{0} \mathrm{~K} \mid \\
\end{array}$ & $\begin{array}{c}\tau \\
\text { Nitrate } \\
\text { Loss } \\
\end{array}$ & Gases Detected \\
\hline HTS + Guanidinium Nitrate $\left(\mathrm{GuNO}_{3}\right)$ & 505 & 8 & \\
\hline HTS + Ethyleneglycol (EG) & 505 & 34 & \\
\hline FiTS + Sulfolane & 505 & 12 & \\
\hline HTS + Diethyleneglyicol (DEG) & 505 & 40 & \\
\hline$(\mathrm{LiNaK}) \mathrm{NO}_{3}+$ Formamide & 505 & 14 & \\
\hline$(\mathrm{LiNaK}) \mathrm{NO}_{3}+\mathrm{GuNO}_{3}$ & 505 & 21 & \\
\hline$\left(\mathrm{LiNaK} \mathrm{NO}_{3}+\mathrm{EG}\right.$ & 505 & 28 & \\
\hline$\left(\mathrm{LiNaK}_{1} \mathrm{NO}_{3}+\right.$ Sulfulane & 505 & 23 & \\
\hline$\left(\mathrm{NaKCa}_{\mathrm{a}} \mathrm{NO}_{3} \cdot+\mathrm{H}_{2} \mathrm{O}+\mathrm{GuNO}_{3}\right.$ & 505 & 12 & \\
\hline$\left(\mathrm{NaKCa}_{2}\right) \mathrm{NO}_{3} \cdot 4 \mathrm{H}_{2} \mathrm{O}+$ Acetamide & 505 & 38 & \\
\hline$(\mathrm{NaKC}) \mathrm{NO}_{3}+$ Acetamide & 533 & 35 & $\mathrm{CH}_{4}, \mathrm{CO}, \mathrm{CO}_{2}, \mathrm{NO}_{x}$ \\
\hline $\mathrm{NaKCa} \mathrm{NO}_{3} \cdot 4 \mathrm{H}_{2} \mathrm{O}+\mathrm{EG}$ & 505 & 32 & \\
\hline$(\mathrm{NaKCa}) \mathrm{NO}_{3}+$ Dimethylformamide & 505 & 45 & $\mathrm{H}_{2} . \mathrm{CH}_{4}, \mathrm{CO}, \mathrm{CO}_{2}, \mathrm{NO}_{\mathrm{x}}$ \\
\hline$\left(\mathrm{NaKCa} \mathrm{NO}_{3}+\right.$ Sulfolane & 505 & 31 & $\mathrm{CO}_{2}, \mathrm{NO}_{\mathrm{x}}$ \\
\hline$\left(\mathrm{NaKCa}_{\mathrm{a}}\right) \mathrm{NO}_{3}+$ Sulfolane & . 533 & 57 & \\
\hline$\left(\mathrm{NaKC}_{3}\right) \mathrm{NO}_{3} \cdot 4 \mathrm{H}_{2} \mathrm{O}+\mathrm{DEG}$ & 505 & 25 & $\cdot$ \\
\hline$(\mathrm{NaKCa}) \mathrm{NO}_{3}+\mathrm{M}-\mathrm{Pyrol}_{\mathrm{r}}$ & 533 & 50 & $\mathrm{CO}_{2}, \mathrm{NO}_{\mathrm{X}}$ \\
\hline$\left(\mathrm{NaKCa}_{3} \mathrm{NO}_{3}+\right.$ DEGDME & 533 & 40 & $\cdot$ \\
\hline$(\mathrm{NaKCa}) \mathrm{NO}_{3}+\mathrm{DEG} M E E$ & 533 & 60 & \\
\hline$(\mathrm{NaKCa}) \mathrm{NO}_{3}+$ Triethyleneglycol & 533 & 43 & \\
\hline$(\mathrm{NaKCa}) \mathrm{NO}_{3}+$ Phthalonitrile & 533 & 7 & \\
\hline$\left(\mathrm{NaKC}_{3}\right)_{\mathrm{NO}_{3}}+$ Phthalimide & 533 & 13 & \\
\hline$(\mathrm{NaKCa}) \mathrm{NO}_{3}+$ Dibutylphthalate & 533 & 10 & \\
\hline$\left(\mathrm{NaKCaNO}_{3}+\right.$ Triphenylphosphate & 533 & 55 & $\mathrm{CO}, \mathrm{CO}_{2} \cdot \mathrm{NO}_{\mathrm{X}}$ \\
\hline$\left(\mathrm{NaKCa}_{3}\right) \mathrm{NO}_{3}$ only & 533 & 16 & \\
\hline
\end{tabular}

The initial sample size is $4 \mathrm{~g}$ 
ANALYTICAL RESULTS OF POST-TEST THERIIAL STABILITY SAMPLES 6 HOURS AT $505^{\circ} \mathrm{K}$

\begin{tabular}{|c|c|c|c|c|}
\hline Sample & $\begin{array}{c}\ddot{\prime} \\
\text { Nitrate } \\
\text { Loss } \\
\end{array}$ & 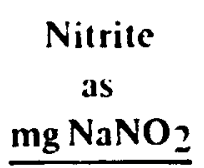 & $\begin{array}{c}\text { Carbonate } \\
\text { as } \\
\mathrm{mgCO}_{2}\end{array}$ & $\begin{array}{c}\text { Gases } \\
\text { Detected } \\
\end{array}$ \\
\hline$(\mathrm{NaKCa}) \mathrm{NO}_{3}+$ Acetamide & 3.4 & 0.004 & 4.3 & $\mathrm{CO}_{2} \mathrm{NO}_{\mathrm{x}}$ \\
\hline$\left(\mathrm{NaKC}_{\mathrm{a}}\right) \mathrm{NO}_{3}+$ Ethyleneglycol & 3.4 & 0.036 & 1.6 & $\mathrm{CO} . \mathrm{CO}_{2} . \mathrm{NO}_{\mathrm{X}}$ \\
\hline$(\mathrm{NaKC} a) \mathrm{NO}_{3}+$ Dimethylformamide & 31 & 0.03 & 202.5 & $\mathrm{H}_{2} \cdot \mathrm{CH}_{4} \cdot \mathrm{CO}, \mathrm{CO}_{2}, \mathrm{NO}_{x}$ \\
\hline$\left(\mathrm{NaKCa}_{2}\right)_{3} \mathrm{~N}_{3}+$ Sulfolane & 21 & 0.76 & 58 & $\mathrm{CO} . \mathrm{CO}_{2} . \mathrm{NO}_{\mathrm{X}}$ \\
\hline$\left(\mathrm{NaKCa} \mathrm{NO}_{3}+\right.$ Phthalimide & 0 & 0.003 & 0.5 & $\mathrm{CO}_{2}, \mathrm{NO}_{\mathrm{X}}$ \\
\hline$(\mathrm{NaKC} a) \mathrm{NO}_{3}+$ Dimethylphthalate & 0 & 0.80 & $\cdots$ & $\mathrm{CO}^{-\mathrm{CO}_{2}} \mathrm{NO}_{\mathrm{X}}$ \\
\hline$(\mathrm{NaKCa}) \mathrm{NO}_{3}+4.4 \cdot$ Thiodiphenol & 0 & 0.084 & - & $\mathrm{CO}_{2} \mathrm{NO}_{\mathrm{X}}$ \\
\hline$\left(\mathrm{NaKC}_{\mathrm{a}}\right) \mathrm{NO}_{3}+$ Triphenylphosphate & 9.4 & 0.80 & 4.1 & $\mathrm{CO} . \mathrm{CO}_{2} . \mathrm{NO}_{\mathrm{x}}$ \\
\hline
\end{tabular}




\section{RESULTS OF MATERIALS COMPATIBILITY TESTS AT $505^{\circ} \mathrm{K}$ FOR 24 HOURS}

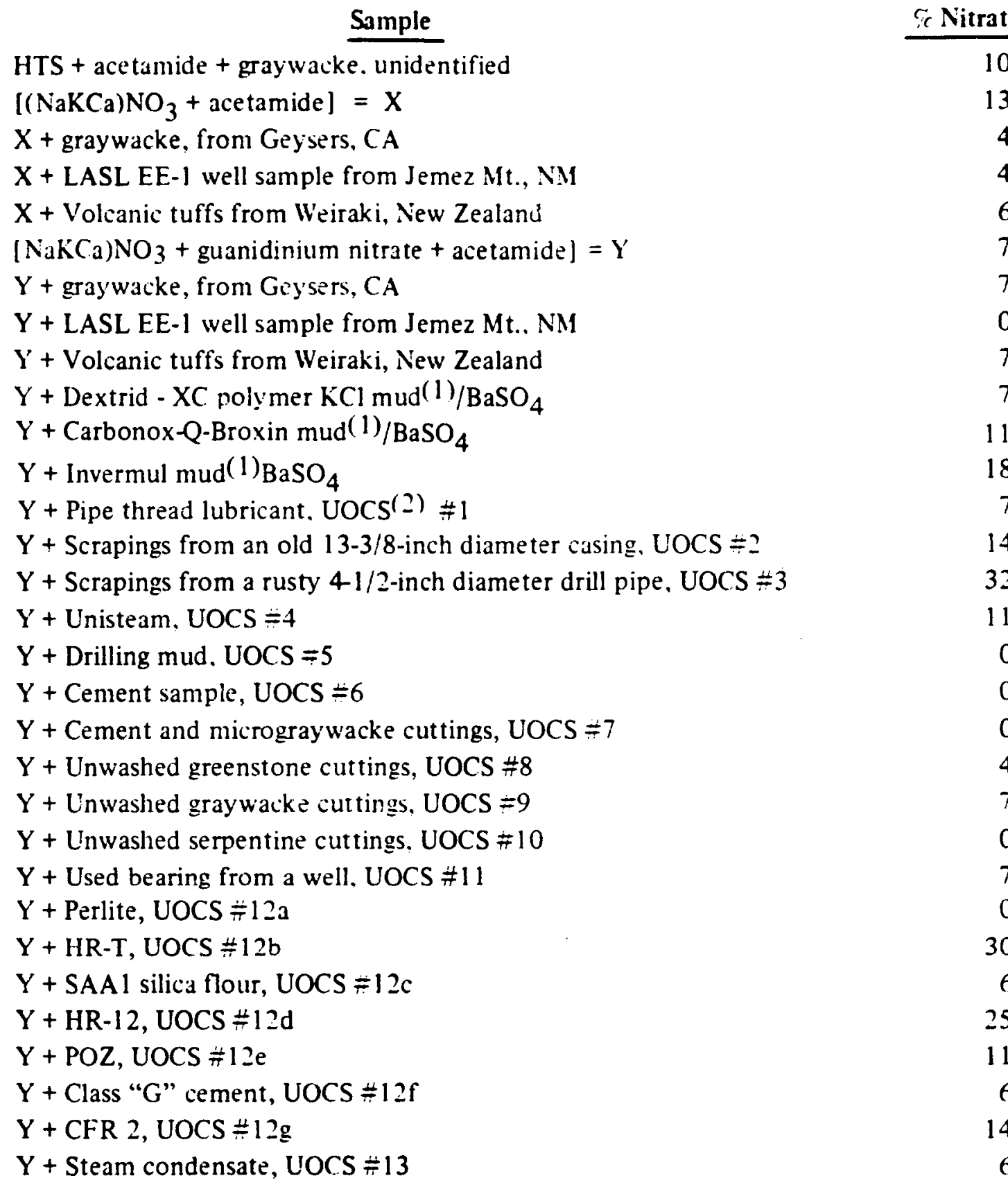

(1 These mud samples were obtained fron $\mathrm{Dr}$. A. Spencer. They were combined with $20 \%$ by weight $\mathrm{BaSO}_{4}$.

$\therefore$ UOCS = Union Oil Company Sample. These samples were obtaired from Union Geothermal Division of Union Oil Company of California, lociared in Santa Rosa. Additional details of these samples can be found in the correspondence from Jack Hartz of Union Oil Company to Dr. E. Schmidt of ROCKCOR, dated September 21. 1977. 


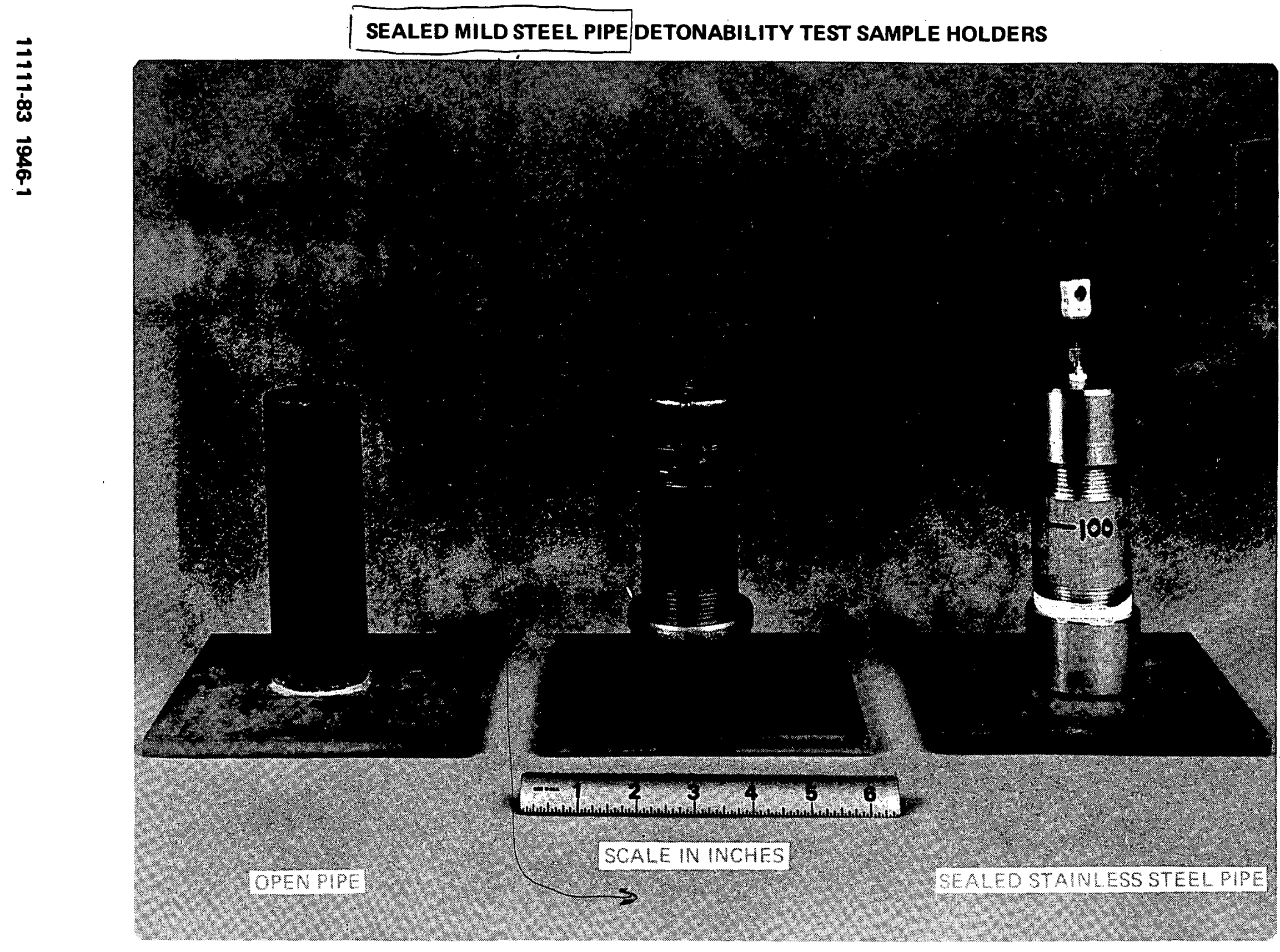




\section{6mm DIAMETER DETONABILITY TESTS BEFORE AND AFTER DETONATION}

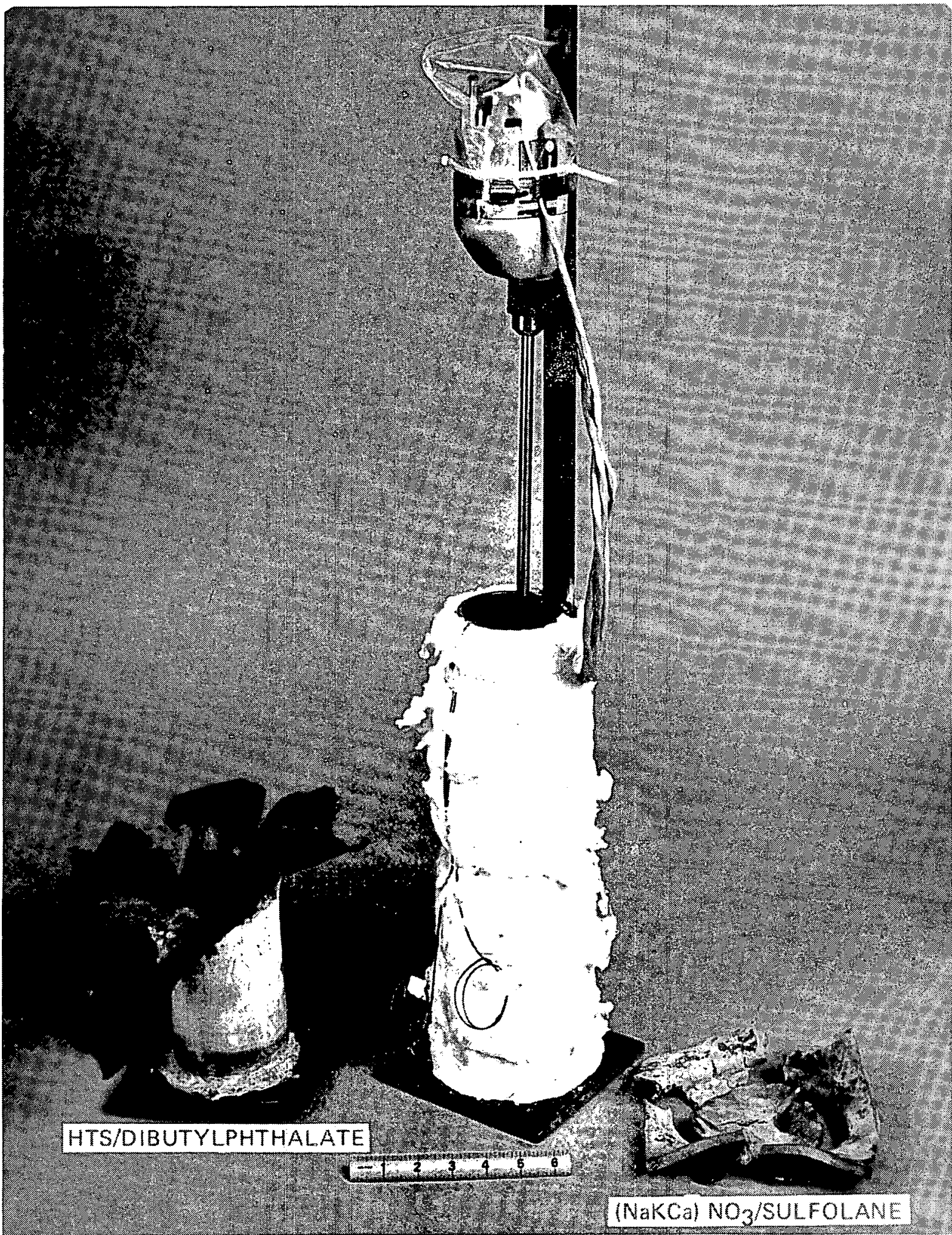




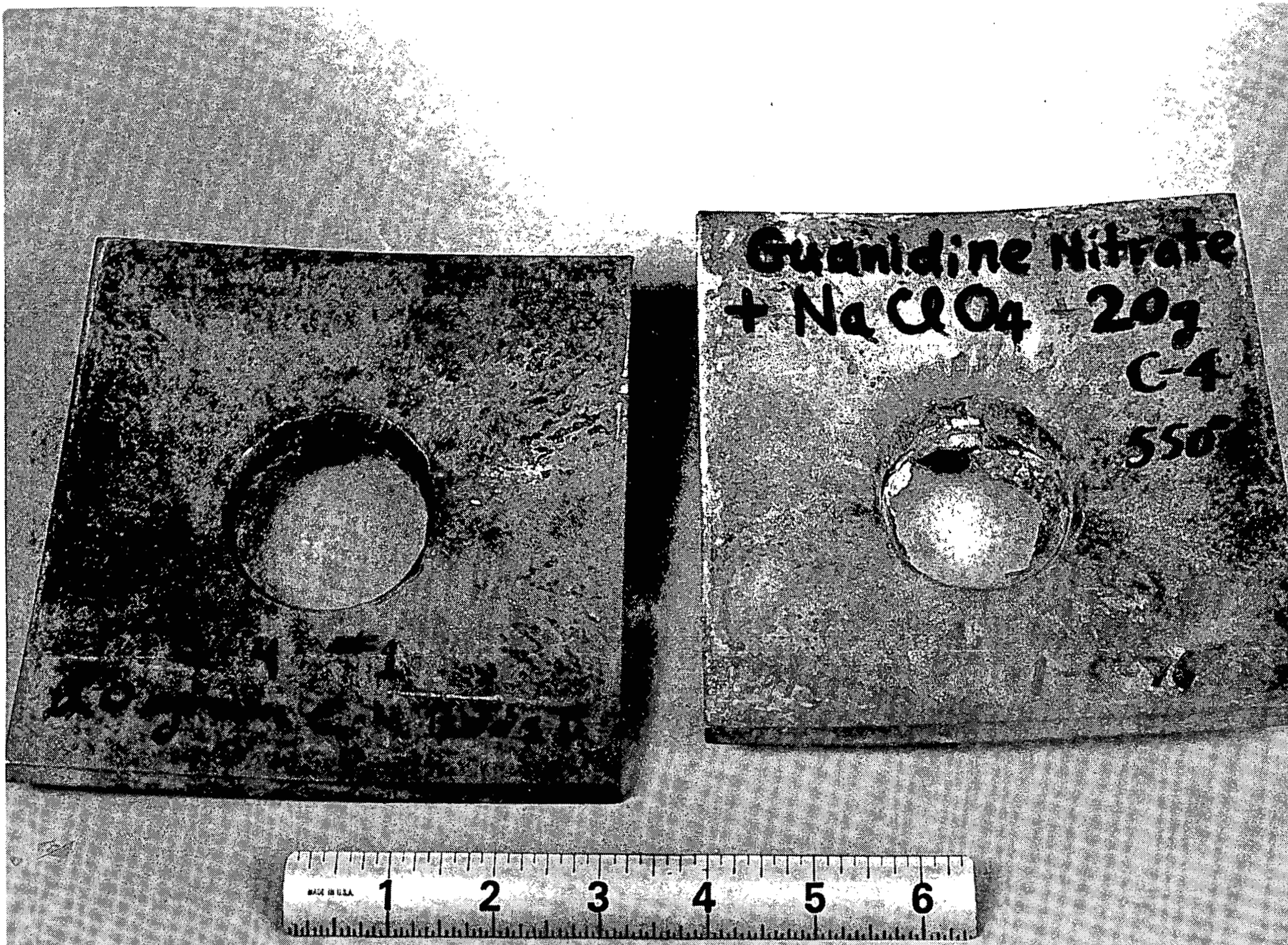

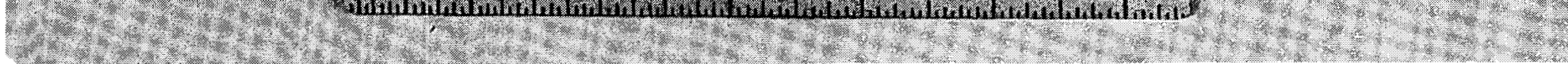


DETONATION TEST RESULTS - OPEN PIPE TESTS

\begin{tabular}{|c|c|c|c|c|c|}
\hline \multirow{2}{*}{ Oxidizer } & \multirow{2}{*}{ Fuel } & \multicolumn{2}{|c|}{ Temperature } & \multirow{2}{*}{ Iniliation } & \multirow{2}{*}{$\begin{array}{l}\text { Wituess Plate } \\
\text { Indenlationn } \\
\text { (muin) }\end{array}$} \\
\hline & & ${ }^{\prime} K$ & $\omega_{F}$ & & \\
\hline$(\cdot 4$ & $c-4$ & 293.3 & 77 & Cap & $\infty$ \\
\hline PTE'-4' & PTC 4 & 29.3 & 77 & $\left(j p+c-4^{2}\right.$ & $\infty$ \\
\hline $\mathrm{NaC} \mathrm{NO}_{4}$ & Guanidinium nit rate & 561 & 550 & $\mathrm{Cap}+\mathrm{C} \cdot 4$ & $\infty$ \\
\hline $\mathrm{NaClO}_{4} \cdot \mathrm{H}_{2} \mathrm{O}$ & Triethylene glycol & 552 & 5.34 & Autodelonated & $\infty$ \\
\hline$\left(\mathrm{NaK}(\mathrm{a}) \mathrm{NO}_{3}\right.$ & Guanidinium nitrate & $5 n 1$ & 550 & Cap & $\infty$ \\
\hline$\left(\mathrm{NaK}\left(\mathrm{C}^{\mathrm{a}}\right) \mathrm{NO}_{3}\right.$ & Acetumide & 480 & 405 & Cap + C.4 & $37^{3}$ \\
\hline $\mathrm{N}: 1 \mathrm{C}^{\prime} \mathrm{IO}_{4}$ & Diethylene glycol & 519 & 475 & $C a p+C \cdot 4$ & $.30^{4}$ \\
\hline $\mathrm{N}: \mathrm{ClO}_{4}$ & Ethylene glycol & $4(09$ & $3 \times 5$ & $\mathrm{Cap}+\mathrm{C} \cdot 4$ & $2 x^{4}$ \\
\hline$(\mathrm{NaKCa}) \mathrm{NO}_{3} \cdot 4 \mathrm{H}_{2} \mathrm{O}$ & Sulfolune & 533 & 500 & Cap & $2 x^{4}$ \\
\hline$(\mathrm{IiNaK}) \mathrm{N}()_{3}+\mathrm{NaClO}_{4}$ & (ilycerol & 555 & 540 & $\mathrm{Can}+\mathrm{C}-4$ & 25 \\
\hline $\mathrm{INO}_{3}+\mathrm{H}_{2} \mathrm{O}$ & Nitrobenzene & 29.3 & 77 & Cap & 25 \\
\hline $\mathrm{N}: \mathrm{IClO}_{4}$ & lithylene flycol & $47 !$ & 390 & Cap & 104 \\
\hline $\mathrm{Nu}\left(\mathrm{ClO}_{4}\right.$ & Dicthylene glycol & 511.5 & 450 & (ap) & $1 x$ \\
\hline $\mathrm{NaClO}_{4}$ & Guanidinium nit rate & 501 & 550 & Cop & 17 \\
\hline$(\mathrm{LiNaK}) \mathrm{NO}_{3}$ & Ethylene glycol & 477 & 400 & $\mathrm{Cap}+\mathrm{C}-4$ & 17 \\
\hline$\left(\mathrm{NaK}(\mathrm{a}) \mathrm{NO}_{3} \cdot 4 \mathrm{H}_{2} \mathrm{O}\right.$ & Triplicily/plonspliale & 5.33 & 500 & Cap & 16 \\
\hline$\left(\mathrm{NaK}(\cdot \mathrm{a}) \mathrm{N}()_{3} \cdot 4 \mathrm{H}_{2}()+\mathrm{NaClO}_{4}\right.$ & Dibuty|pluthalate & 501 & 550 & Cap & 14 \\
\hline $\mathrm{N}: \mathrm{ClO}_{4} \cdot \mathrm{H}_{2} \mathrm{O}$ & Acetimmide & 505 & 450 & Cup & 1.3 \\
\hline$(\mathrm{l} i \mathrm{NaK}) \mathrm{NO}_{3}+\mathrm{NaClO}_{4}$ & (ilycerol & 561 & 550 & Cap & 13 \\
\hline Nunc & m-Dinitrobenzene & 561 & 550 & Cap & 12 \\
\hline $\mathrm{KClO}_{4}$ & m-Dinitruben/ene & 550 & 5.30 & Cap & 10 \\
\hline $111 s^{5}$ & Melamine & 501 & 550 & (ap & 10 \\
\hline $\mathrm{HISS}+\mathrm{NaClO})_{4}$ & Dihuly|phulaslate & $5.3 .3+$ & $50(0)+$ & (alp & 9 \\
\hline
\end{tabular}


DETONATION TEST RESULTS - OPEN PIPE TLSTS

\begin{tabular}{|c|c|c|c|c|c|}
\hline \multirow{2}{*}{ Oxidizer } & \multirow{2}{*}{ Fuel } & \multicolumn{2}{|c|}{ Temperature } & \multirow{2}{*}{ Initiation } & \multirow{2}{*}{$\begin{array}{c}\text { Witness Plate } \\
\text { Indentation } \\
\text { (num) }\end{array}$} \\
\hline & & $\mathbf{o}_{\mathbf{K}}$ & $\mathbf{o}_{\mathbf{F}}$ & & \\
\hline$(\mathrm{N}: 1 K(\mathrm{a}) \mathrm{NO})_{3} \cdot 4 I_{2} \mathrm{O}$ & Acelamide & 5015 & 450 & Cap & $x$ \\
\hline$(\mathrm{l} . \mathrm{iNaK}) \mathrm{NO}_{3}$ & Diethyletie glycol & 505 & 450 & (ap & $x$ \\
\hline IITS & Dimethylphthalate & 550 & 530 & Cap & 7 \\
\hline Nonce & 2,4-1Dinitrotolucic & 522 & 480 & $\mathrm{Cap}+\mathrm{C}-4$ & 7 \\
\hline Nime & 2,4-1)initrotoluene & 501 & 550 & (ap & 0 \\
\hline$(\mathrm{LiNaK}) \mathrm{NO}_{3}$ & Glycerol & 544 & 520 & Cap & 6 \\
\hline Nomle & m-Dinilrobenzene & 547 & 525 & $\mathrm{Cap}+\mathrm{C}-4$ & 5 \\
\hline ( NaK('i) $\mathrm{NO}_{3}$ & Silicone vil & 505 & 450 & Cap & 5 \\
\hline IITS & Dihutylphthlialate & $5(, 1$ & .550 & (a) & .4 \\
\hline IITS & l:iliylence glycol & 406 & .380 & Cap & 3 \\
\hline IITS & Dowtlierm G & 533 & 500 & Cap + C.4 & 3 \\
\hline IITS & Dihutylphthulate & 561 & 5.50 & $\mathrm{Cap}+\mathrm{C}_{-4}$ & 3 \\
\hline IITS & Acelamide & 505 & 450 & cap & 1 \\
\hline HTS & Dibutylphithalate & 293 & 77 & Cup & Nome \\
\hline IITS & Glycerol & 477 & 400 & Cap & Nonle \\
\hline IITS & Formamide & 477 & 400 & Cup & None \\
\hline IITS & Mineral oil & 505 & 450 & Cap & Nonc \\
\hline IITs & Dowlherm $\mathrm{C}$ & 53.3 & 500 & C:ip & Nom: \\
\hline Ms & Therminol 55 & 50.1 & 550 & $\left(a_{i}\right)$ & Nince \\
\hline HTS & Tiphenylphosphate & 561 & 550 & Cap & Nence \\
\hline HTs & Sulfolanc & 5.33 & 500 & Cap & Nome \\
\hline$\left(\right.$ liNaK) $\mathrm{NO}_{3}$ & lithylene glycol & 472 & 390 & ciap & None: \\
\hline$(\mathrm{LiNaK}) \mathrm{NO}_{3}$ & Acetamide & 505 & 4.50 & Cap & Notle \\
\hline
\end{tabular}


DETONATION TEST RLSULTS - OPLN PIPE TESTS

\begin{tabular}{|c|c|c|c|c|c|}
\hline \multirow{2}{*}{ Oxidizer } & \multirow{2}{*}{ Fuel } & \multicolumn{2}{|c|}{ Temperature } & \multirow{2}{*}{ Initiation } & \multirow{2}{*}{$\begin{array}{c}\text { Witness Plate } \\
\text { Indentation } \\
\text { (mm) }\end{array}$} \\
\hline & & $o_{K}$ & oF & & \\
\hline$(I . \mathrm{NaK}) \mathrm{N}()_{3}$ & Dihutylphullaliale & 56,1 & 550 & Cap & Nome \\
\hline$(\mathrm{l} . \mathrm{iNaK}) \mathrm{NO}_{3}$ & Sultulane & 5.33 & 500 & Cap & Nomick \\
\hline$(\mathrm{LiNaK}) \mathrm{NO}_{3}+\mathrm{NaClO}_{4}$ & Dibutylphillualate & 561 & 550 & Cap & Nomic \\
\hline$\left(\mathrm{NaKCa}^{\prime} \mathrm{NO}_{3} \cdot 4 \mathrm{H}_{2} \mathrm{O}\right.$ & Dibutylphthalate & 561 & 550 & Cap & Nonc: \\
\hline$\left(\mathrm{NaK}(\cdot a) \mathrm{NO}_{3} \cdot 4 \mathrm{I}_{2} \mathrm{O}\right.$ & Dimethylphthalate & 550 & 5.30 & cap & Nomico \\
\hline$(\mathrm{NaKC}$ a $) \mathrm{NO}_{3} \cdot 41_{2} \mathrm{O}$ & Therminol or & 561 & 550 & Cap & Nonce \\
\hline$(\mathrm{NaKC} \cdot \mathrm{a}) \mathrm{NO}_{3}$ & Acetamide & 293 & 77 & Cap & Nente \\
\hline$(\mathrm{NaKCa}) \mathrm{NO}_{3}$ & Guanidinium nitrate + acetamide $(70 / 30)$ & 297 & 75 & Cap & Nonic \\
\hline $\mathrm{NaClO}_{4}$ & Melamine & 29.3 & 77 & Cap & Nont: \\
\hline Nomle & m-Dinitrohen/ene & 37.3 & 212 & Cap & Nom: \\
\hline Numt & (iuanidinium uiltate $+\mathrm{H}_{2} \mathrm{O}$ & 29.3 & 77 & Cap & None \\
\hline
\end{tabular}

ANNOTATIONS:

1 PTC'-4 .. Proprictary explosive of Pelroleum Techuology Corporation, a subsidiary of ROCKCOR. Inc.

2 A llercules No. 8 blasting cap and $20 \mathrm{~g}$ booster charge of composition $C-4$.

3 This was a $1-5 / 8$ " ( dia $)$ x 12 " open pipe bomb containing slightly over one pound of the explosive mixture. All the others in the 1 able were $100 \mathrm{~g}$ mixtures.

4 Witness plate penctrated, but not to the extent that a high order detomation occurred.

$5 \quad$ IITS $=$ heat treatment sult $=\mathrm{KNO}_{3}: \mathrm{NaNO}_{3}: \mathrm{NaNO}_{2}(53: 7: 40 \%$ by weight $)$.

6 The explosion was somewhal louder than just the blasting cap.

NOTI: All inmiscible nixtures contained $2 \%$ (ab-O-Sil and were stirred while hot, just prior to intiation, by a remotely activated stitrer. 


\section{DETONABILITY TEST RESLLTS OF SOME IIIXTURES OF GUANIDINIUM NITR ATE AND ACET AMIDE}

\begin{tabular}{|c|c|c|c|c|}
\hline \multirow{2}{*}{ Sample ${ }^{1}$} & \multicolumn{2}{|c|}{ Temperature } & \multirow{2}{*}{ Initiation } & \multirow{2}{*}{ Result } \\
\hline & ${ }^{\mathbf{O}_{K}}$ & ${ }^{O F}$ & & \\
\hline $\begin{array}{l}\text { Guanidinium nitrate + acetamide } \\
(65 / 35)^{3}\end{array}$ & 505 & 450 & Cap' & Negative \\
\hline $\begin{array}{l}\text { Guanid inium nitrate }+ \text { acetamide } \\
(75 / 25)\end{array}$ & 505 & 450 & Cap & Negative \\
\hline $\begin{array}{l}\text { Guanidinium nit rate }+ \text { acetamide } \\
(85 / 15)\end{array}$ & 505 & 450 & Cap & Negative \\
\hline Guanidinium nitrate & 505 & 450 & Cap & Negative \\
\hline $\begin{array}{l}\text { Guanidinium nitrate }+ \text { acetamide } \\
(70 / 30) 4\end{array}$ & $477+$ & $400+$ & $\mathrm{Cap}+\mathrm{C}+(20 \mathrm{~g})$ & Negative \\
\hline
\end{tabular}

${ }^{1}$ Unless otherwise specified, these were $80 \mathrm{~g}$ samples in $37 \mathrm{~mm}$ diameter pipes

2 Hercules No. 8 detonator squib

3 The numbers in parentheses indicate percent by wejght of each, respectively

4 This was a $5 \mathrm{lb}$ mixture. It was tested in a 3 -i:ch diameter pipe 
COOK-OFF TEST RESULTS'

\begin{tabular}{|c|c|c|c|c|c|c|c|}
\hline \multirow{2}{*}{ Senmple } & \multicolumn{2}{|c|}{$\begin{array}{l}\text { Average Ilealing } \\
\text { Rale }\end{array}$} & \multirow{2}{*}{ Result } & \multirow{2}{*}{ Exothem } & \multicolumn{2}{|c|}{$\begin{array}{l}\text { Heating Rate at } \\
\text { Exotherm Temp. }\end{array}$} & \multirow{2}{*}{$\begin{array}{c}\text { DSC } \\
\text { Exotherm2 }\end{array}$} \\
\hline & $\theta_{K} / \mathrm{Inin}$ & OF/min & & & oK/min & $0 F / m i n$ & \\
\hline 1. ( $\mathrm{NaK}(\mathrm{a}) \mathrm{NO}_{3}+$ acetamide & 5.1 & 9.2 & Blew top at $6.390 \mathrm{~K}\left(690^{\circ} \mathrm{F}\right)$ & $586^{\circ} \mathrm{K}\left(596^{\circ} \mathrm{F}\right)$ & 2.6 & 4.6 & $5.370 \mathrm{~K}$ \\
\hline$\therefore\left(\mathrm{N}_{\mathrm{a}} \mathrm{K}\left({ }^{\circ} \mathrm{a}\right) \mathrm{NO}_{3}+\right.$ phithulimide & 5.6 & 10.0 & Blew top al $575^{\circ} \mathrm{K}\left(575^{\circ} \mathrm{F}\right)$ & $5.37^{\circ} \mathrm{K}\left(50 \mathrm{KOF}^{\circ}\right)$ & 1.4 & 2.5 & $5.36^{(1)} \mathrm{K}$ \\
\hline 3. $\left(\mathrm{NaK}_{\mathrm{K}}(\mathrm{a}) \mathrm{NO}_{3}+\right.$ sulliblame & 7.5 & 1.3 .5 & Dellagrated at $58.5^{\circ} \mathrm{K}\left(504^{\circ} \mathrm{F}\right)$ & $54.30 \mathrm{~K}(5180 \mathrm{~F})$ & 5.9 & 10.7 & $4100 \mathrm{~K}$ \\
\hline 4. $\left(N_{a} K(\right.$ a) $) N_{3}+$ dimellıylformuanide & 6.4 & 11.5 & Blew (op) at $5250 \mathrm{~K}(4850 \mathrm{li})$ & $5160 \mathrm{~K}(470 \mathrm{in})$ & 2.0 & 3.7 & $4 \times 4() \mathrm{K}$ \\
\hline 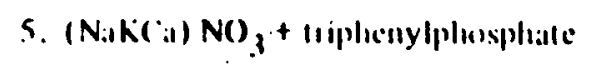 & 6.5 & 11.7 & IHlew lop at $5 \times 10 \mathrm{~K}(58(0) \mid \mathrm{l})$ & $55.3(1) R(5.36(1) 1)$ & 5.6 & 10.0 & $54211 \mathrm{~K}$ \\
\hline 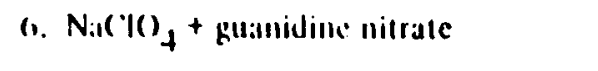 & 3.0 & 5.4 & Hlew lop al $744^{\circ} \mathrm{K}\left(880^{\circ} \mathrm{F}\right)$ & $56.31) \mathrm{K}(.554 \cdot 1)$ & 0.6 & 1.0 & $S 6 K(1)$ \\
\hline 7. $\left(\mathrm{N}: 1 \mathrm{~K}(\mathrm{a}) \mathrm{N}()_{3}+\mathrm{M}-\right.$ pyrol & 0.2 & 11.0 & Burst at $51 \mathrm{I}^{0 \mathrm{~K}}(4600 \mathrm{~F})$ & SOS"K (450') & Unk & wil & $495.5 .370 \mathrm{~K}$ \\
\hline 8. ( $\mathrm{Nat}\left(\mathrm{K}^{\prime}\right.$ a) $\mathrm{NO}_{3}+$ fuanidine nitrate ${ }^{3}$ & 2.8 & 5.1 & Burst at (6)10K (7850) & $5850 \mathrm{~K}(50.30 \mathrm{~F})$ & 0.94 & 1.7 & $S \times X 11 \mathrm{~K}$ \\
\hline 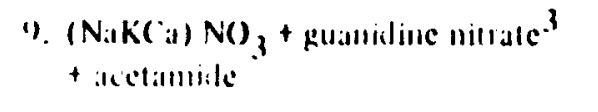 & 3.0 & 5.5 & Burst al 50201 $(606,10)$ & $5680 \mathrm{~K}(56.30 \mathrm{0})$ & $0.7 x$ & 1.4 & $506,1) k$ \\
\hline 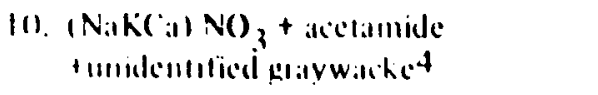 & 5.0 & 9.0 & Burst at $\sim(1,300 \mathrm{~K}(\sim 6000)$ & $5550 \mathrm{~K}\left(540^{\circ}\right)$ & 1.3 & $2 . .3$ & $5.3111 \mathrm{~K}$ \\
\hline
\end{tabular}

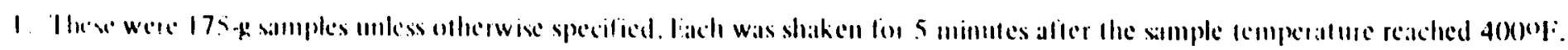

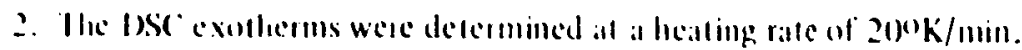

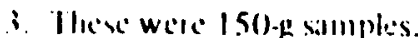

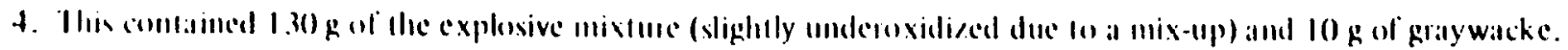


DETONATION TEST RESULTS - SEALED PIPE

\begin{tabular}{|c|c|c|c|c|c|}
\hline \multirow{2}{*}{ Oxiclizer } & \multirow{2}{*}{ Fuel } & \multicolumn{2}{|c|}{ Temperature } & \multirow{2}{*}{ Initiation } & \multirow{2}{*}{ Result } \\
\hline & & $\mathrm{O}_{\mathrm{K}}$ & OF & & \\
\hline$c-4$ & $C-4$ & 293 & 77 & Cap + S.C. ${ }^{1}$ & $\begin{aligned} \text { Positive I High order } \\
\text { I defonation }\end{aligned}$ \\
\hline$P T C-4$ & PTC-4 & 293 & 77 & Cap + S.C. & Pusitive \\
\hline$\left(\mathrm{NaK}(\mathrm{a}) \mathrm{N}()_{3} \cdot 4 \mathrm{H}_{2} \mathrm{O}\right.$ & Formamide? & $>394$ & $>250$ & Cap + S.C. & Negrative. ${ }^{3}$ \\
\hline$(\mathrm{NaK}(\mathrm{a}) \mathrm{NO})_{3} \cdot 41_{2} \mathrm{O}$ & Acctaminide 2 & 505 & 450 & Cap + S.C. & Nequative. ${ }^{3}$ \\
\hline $\mathrm{N}: \mathrm{A}(\mathrm{I}) 4$ & Ciuamidinium nitratte & 550 & 5.30 & Cal + S.C. & Posilive. 3 \\
\hline$(\mathrm{NakCa}) \mathrm{NO}_{3}$ & Dimethylformamicle & 511 & 460 & $(a)+S . C$. & Posilive \\
\hline$\left(N: 1 K(: a) N()_{3}\right.$ & Acetamide & -510 & $\sim 475$ & X-321-K (a) & Positivo \\
\hline$\left(\mathrm{NiK}(\dot{a}) \mathrm{N}()_{3}\right.$ & Acelamide & 516 & 470 & $C a p+S . C^{4}$ & Positive \\
\hline$\left(N: K(\right.$ i) $) N()_{3}$ & Acot:amide & 516 & 470 & (ap) + S.C. & Posilive \\
\hline$\left(N: 1 K(: a) N()_{3}\right.$ & M-l'yrol & 505 & 450 & $(a p)+S C$. & Negill ive \\
\hline$(\mathrm{NaKC} a) \mathrm{NO}_{3}$ & Triellyylene glycol & 494 & 430 & Cap + S.C. & Posilive \\
\hline$\left(\mathrm{N}: \mathrm{K}(\mathrm{a}) \mathrm{N}()_{3}\right.$ & Phllialonitrilc & 511 & 40,0 & Cap + S.C. & Negallive \\
\hline$\left(\mathrm{N}: \mathrm{K}^{\prime}(\mathrm{a}) \mathrm{N}()_{3}\right.$ & Phthalimilc & 5.33 & 500 & Calp + S.C. & Posilive \\
\hline
\end{tabular}

\section{NoTl:s:}

1 A llercules $N_{0}$. 8 blasting cap altached to the groove of a shaped charge. The groove of the shaped charge was filled with approximately $2 \mathrm{~g}$ of $\left({ }^{-4}\right.$. The shaped charge was aimed through the side of the sealed pipe at a $45^{\circ}$ downward angle at the liquid surface.

2 The fuel was sealed in a polyethylene bag.

3 These were 100 g inixtures. All the whers were $175 \mathrm{~g}$ mixtules.

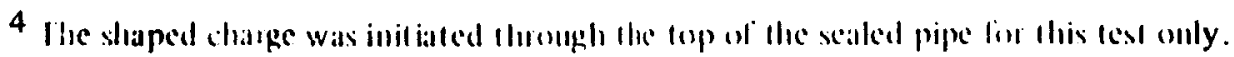




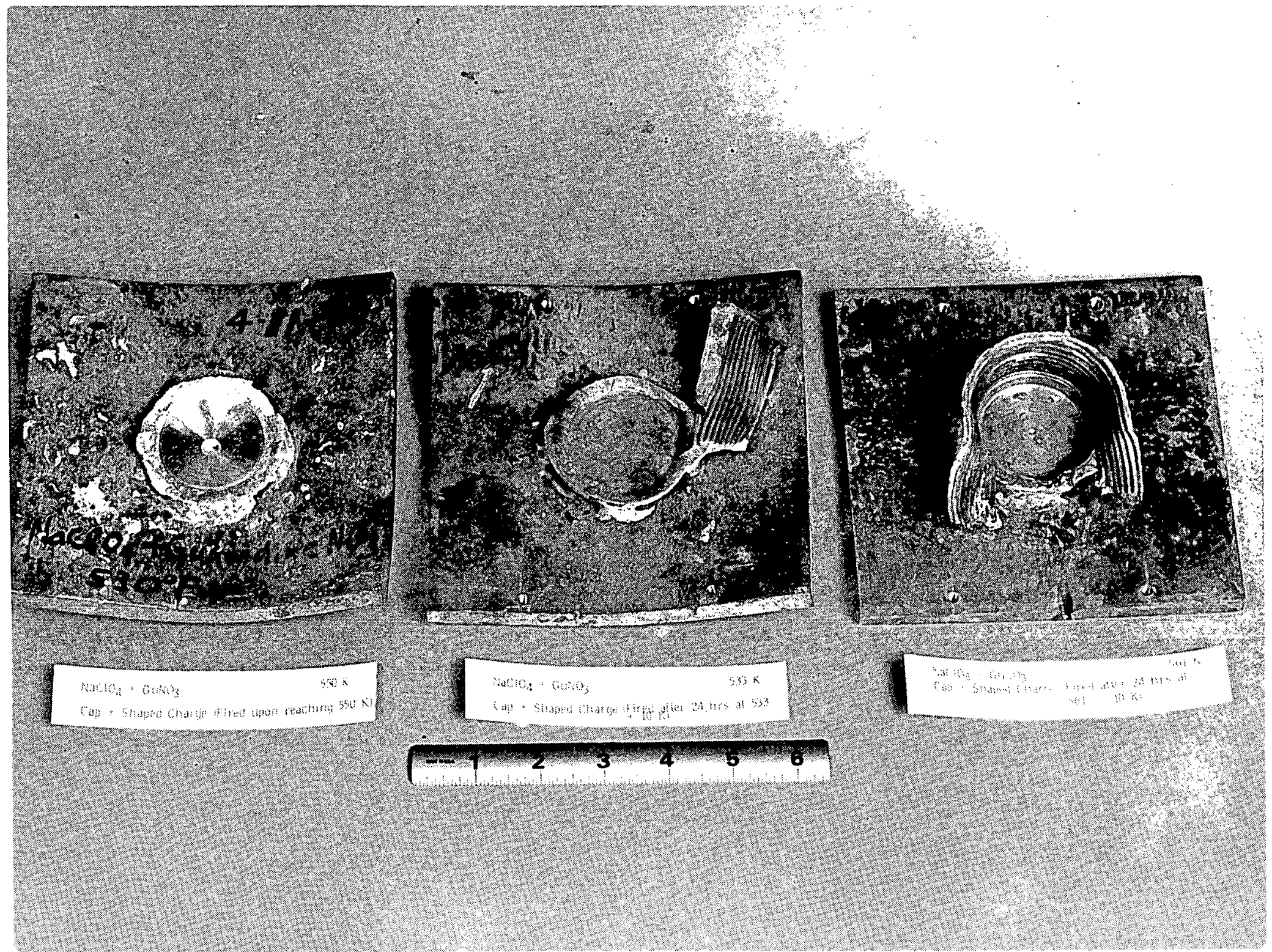




\section{TWENTY-FOUR-HOUR DETONATION TIST RLSULTS}

\begin{tabular}{|c|c|c|c|c|}
\hline \multirow{2}{*}{ S:muple ${ }^{\prime}$} & \multicolumn{2}{|c|}{ Test Tempxeralure } & \multirow{2}{*}{$\begin{array}{c}\text { Duralion } \\
\text { (lurs) }\end{array}$} & \multirow{2}{*}{ Result } \\
\hline & ('K) & (DF) & & \\
\hline 1. $\left.(\mathrm{NaKCa})_{\mathrm{NO}}\right)_{3}+$ acetamide & $491 \pm 10$ & $425+20$ & 2.3 & Detonated on command? \\
\hline 2. ( $\mathrm{NaKCa}) \mathrm{NO}_{3}+$ sulfolanc & $491 \pm 10$ & $425+20$ & 21 & Detonated on command \\
\hline 3. $\mathrm{NaClO}_{4}+$ Cuanidinium nitrate $\left(\mathrm{GuNO}_{3}\right)$ & $533 \pm 10$ & $500 \pm 20$ & 24 & Detonated on command \\
\hline 4. ( NaKCa)NO $3+$ + (riphenylphosplhate & $505 \pm 10$ & $450+20$ & $\therefore 4$ & Negallive \\
\hline 5. (NaK(a)NO 3 + dimethylformamicle & $478+10$ & $4(00)+20$ & 1.3 & Bursl hy ils:ll \\
\hline 6. (NaKCa)NO3 + phthalimide & $505 \pm 10$ & $450 \pm 20$ & 10 & Bursl by itscll \\
\hline 7. $(\mathrm{NaKCa}) \mathrm{NO}_{3}+$ acctamicle & $561 \pm 10$ & $550 ! 20$ & 24 & Burst or dedlagrated ent commmand ${ }^{3}$ \\
\hline 8. $\mathrm{NaClO}_{4}+\mathrm{CiuNO}_{3}$ & $561 \pm 10$ & $550 \pm 20$ & 24 & Burst or deflagrated on command \\
\hline 9. $\left(\mathrm{NaK}^{\prime} \mathrm{Ca}\right) \mathrm{NO}_{3}+\mathrm{GuNO}_{3}$ & $561 \pm 10$ & $550 \pm 20$ & 24 & Burst or defliggraled on command \\
\hline 10. $(\mathrm{NaKCa})+\mathrm{GuNO}_{3}+$ acetamide & $505 \pm 10$ & $450 \pm 20$ & 24 & Delonated on command \\
\hline
\end{tabular}

1 Sample sizes varied from $100 \mathrm{~g}$ (for No. 3 and No. 9), $150 \mathrm{~g}$ (for No. 10$) .10175 \mathrm{~g}$ (for the rest), depending on the ease of loading the
1.5 -inch diameter pipe bounbs.

2 The top and main body of the pipe were hlown into fragments. The threaded holtom cap of the pipe was flated and the witness plate was distorted.

3 The pipe sheared off alove the bottom cap leaving it and the wilness plate cescentially intact with minimal distortion. 
ADIABATIC COMPRESSION TEST APPARATUS

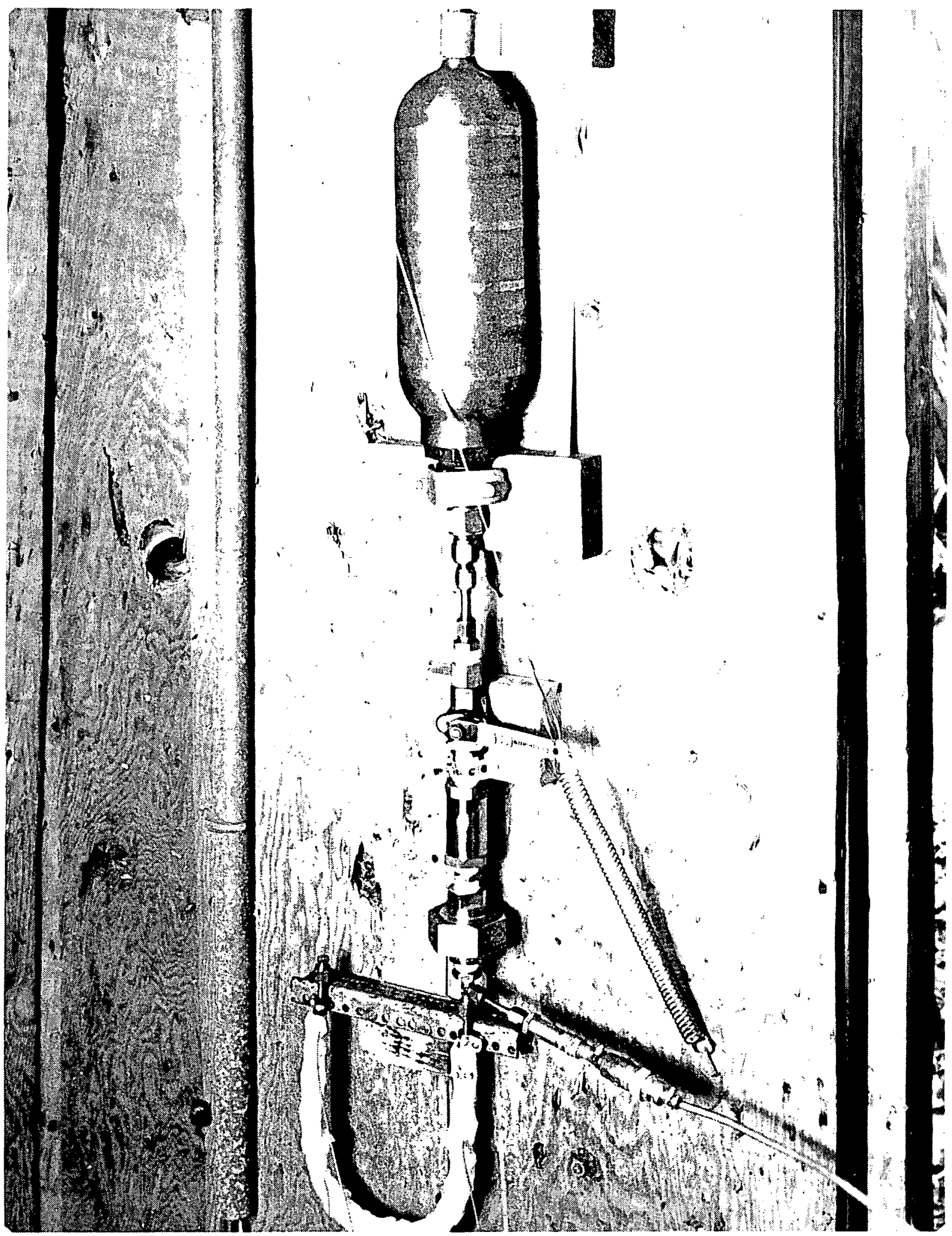


ADIABATIC COMPRESSION TEST RESULTS ${ }^{1}$

\begin{tabular}{|c|c|c|c|c|}
\hline Sample & $\begin{array}{c}\text { Samiple } \\
\text { Size } \\
|g|\end{array}$ & $\begin{array}{c}\text { Temperature } \\
{ }^{\circ} \mathbf{K}\end{array}$ & $\begin{array}{c}\text { Heating } \\
\text { Time } \\
\text { (min) }\end{array}$ & Result \\
\hline $\mathrm{N}_{2} \mathrm{H}_{4}$ & 3.2 & $387\left(238^{\circ} \mathrm{F}\right)$ & 17 & - \\
\hline $\mathrm{N}_{2} \mathrm{H}_{4}$ & 3.2 & $394\left(250^{\circ} \mathrm{F}\right)$ & 14 & - \\
\hline $\mathrm{N}_{2} \mathrm{H}_{4}$ & 4.1 & $422\left(300^{\circ} \mathrm{F}\right)$ & 8.5 & + \\
\hline$(\mathrm{NaKC}) \mathrm{NO}_{3}$ & 3.2 & $533\left(500^{\circ} \mathrm{F}\right)$ & 12.5 & -- \\
\hline $\mathrm{GuNO}_{3}-\mathrm{AcNH}_{2}=$ & 3.2 & $533\left(500^{\circ} \mathrm{Fi}\right.$ & 12 & - \\
\hline$\left(\mathrm{NaKCa}_{\mathrm{a}} \mathrm{NO}_{3}-\mathrm{Gu}_{3} \mathrm{NO}_{3}-\mathrm{AcNH}_{2}{ }^{3}\right.$ & 3.2 & $550\left(530^{\circ} \mathrm{F}\right)$ & 20 & + \\
\hline$(\mathrm{NaKCa}) \mathrm{NO}_{3}-\mathrm{GuNO}_{3}-\mathrm{AcNH}_{2}{ }^{3}$ & 3.2 & $533\left(500^{\circ} \mathrm{F}\right)$ & 8.5 & - \\
\hline$\left(\mathrm{NaKCaNO}_{3}-\mathrm{Gu}_{2} \mathrm{NO}_{3}-\mathrm{AcNH}_{2}{ }^{3}\right.$ & 4.1 & $561\left(550^{\circ} \mathrm{F}\right)$ & 15 & - \\
\hline$\left(\mathrm{NaKCa}_{3}-\mathrm{GuNO}_{3}-\mathrm{AcNH}_{2}{ }^{3}\right.$ & 4.1 & $568\left(563^{\circ} \mathrm{F}\right)$ & 15.5 & - \\
\hline
\end{tabular}

${ }^{1}$ Each sample was suddenly compressed with $2.000 \mathrm{psi}$ of $\mathrm{N}_{2}$ within $3 \mathrm{~ms}$. at an alerage rate of $670.000 \mathrm{psi}$ is

2 This is 70 to $30 \%$ by weight mixture of guanidinium nitrate and acetamide. respectively.

3 This is a $58.7 \%-28.9 \%-12.4 \%$ by weight mixture. The mixture was premolien in a test tube and later transferred to the sample U-tube. 


\section{ELECTROSTATIC DISCHARGE (ESD) TEST RESULTS}

Sample conditions: $\quad$ Molten. temperature $400^{\circ} \mathrm{F}$

Test conditions: $\quad$ Room temperature $-70^{\circ} \mathrm{F}$

Relative humidity $-15 \%$

\begin{tabular}{|l|c|c|}
\hline $\begin{array}{c}\text { Test Level } \\
\text { (Joules) }\end{array}$ & $\begin{array}{c}\text { No. of } \\
\text { Shots }\end{array}$ & $\begin{array}{c}\text { Total No. } \\
\text { of Trials }\end{array}$ \\
\hline 5.0 & 10 & 10 \\
1.26 & 8 & 10 \\
0.50 & 4 & 10 \\
0.075 & 0 & 20 \\
\hline
\end{tabular}

FRICTION SENSITIVITY TEST RESULTS

Sample conditions: Solid

Test conditions: $\quad$ Temperature $-66^{\circ} \mathrm{F}$

Relative humidity $-61^{\circ} \mathrm{F}$

Steel components

\begin{tabular}{|c|c|c|c|}
\hline $\begin{array}{c}\text { Test } \\
\text { Velocity } \\
\text { (mps) }\end{array}$ & $\begin{array}{c}\text { Energy } \\
\text { Level } \\
\left(\mathbf{N} / \mathrm{m}^{2}\right)\end{array}$ & $\begin{array}{c}\text { No. of } \\
\text { Shots }\end{array}$ & $\begin{array}{c}\text { Total No. } \\
\text { of Trials }\end{array}$ \\
\hline 3.0 & $12.1 \times 108$ & 1 & 18 \\
& $10.2 \times 108$ & 1 & 10 \\
& $8.8 \times 108$ & 1 & 10 \\
& $7.5 \times 108$ & 0 & 20 \\
2.4 & $9.9 \times 108$ & 0 & 20 \\
\hline
\end{tabular}

NOTE: LIRA inrared analyzer used to detect reaction.

For the 2.4 mps friction test. $9.9 \times 10^{8} \mathrm{~N} / \mathrm{m}^{2}$ was the highest test level attainable consistent with a proper test.

TIL is $7.5 \times 10^{8} \mathrm{~N} / \mathrm{m} 2$ for $3 \mathrm{mps}$ triction.

TIL is greater than or equal in $9.9 \times 10^{8} \mathrm{~N}$ m- for 2.4 mps trietion.

Friction sample was 0.080 " thick sheet. 


\section{DETONATION ARREST TEST FIXTURE}

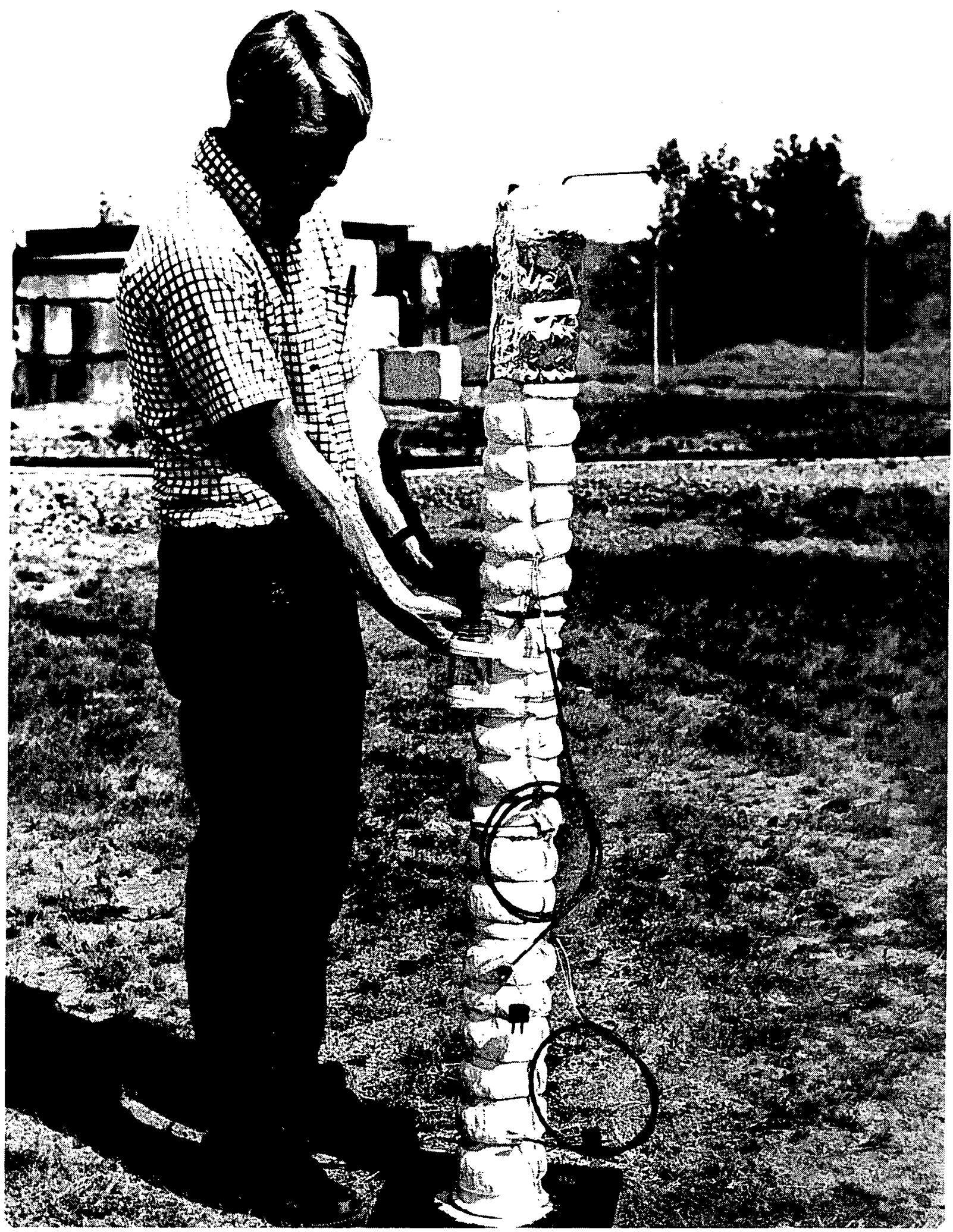




\section{CROSS SECTION OF DETONATION ARREST TEST FIXTURE AT DONAR}

INTERFACE ACCEPTOR

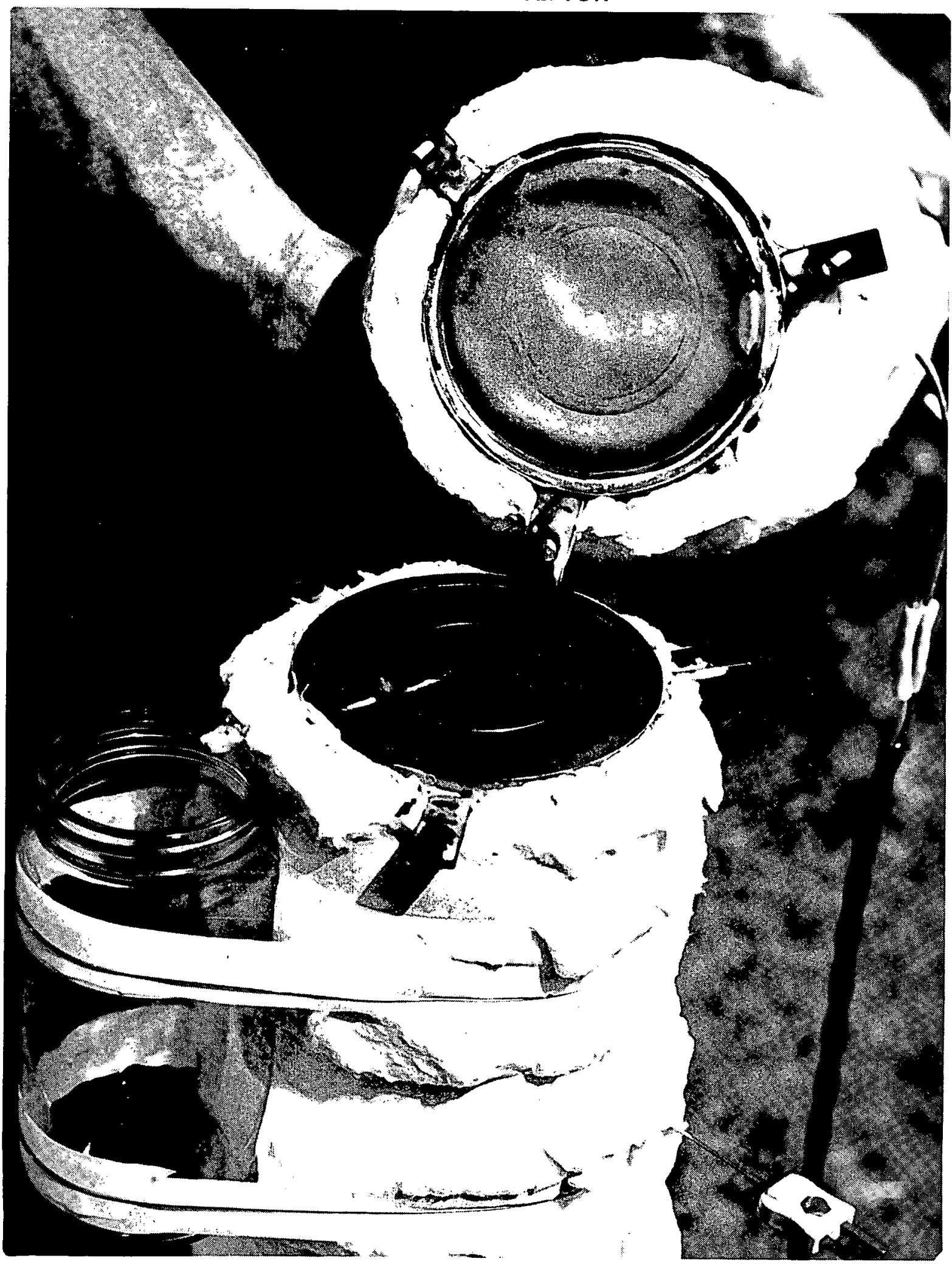




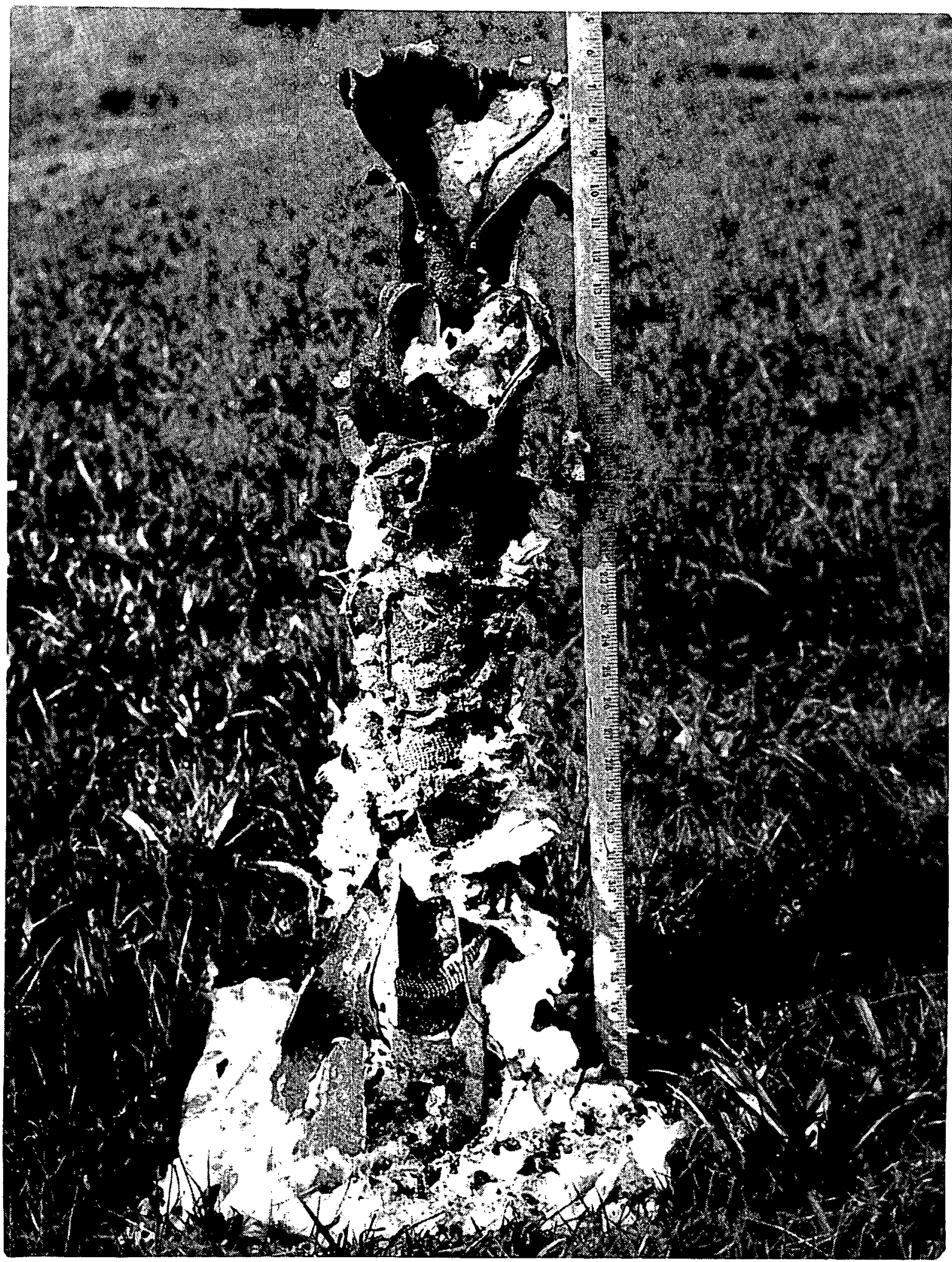




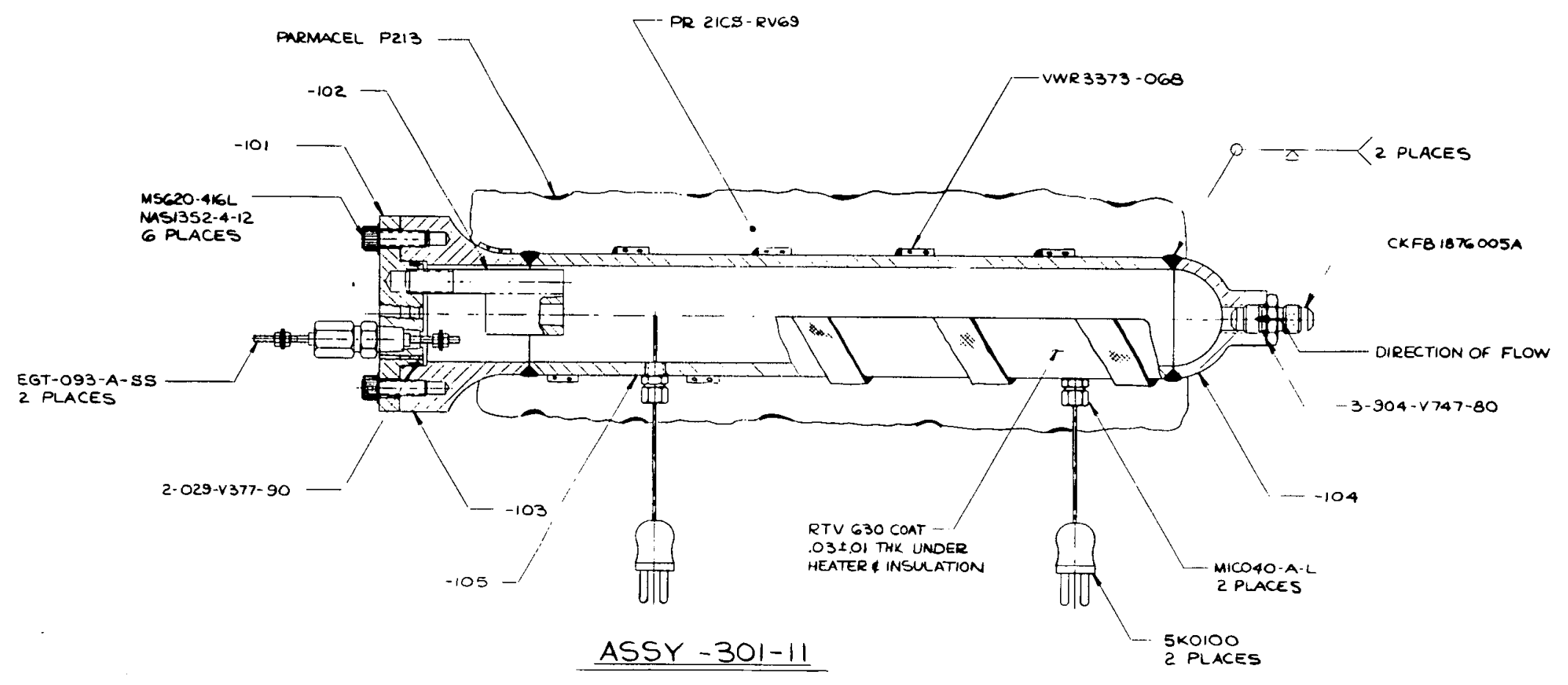




\section{HIGH PRESSURE DETONABILITY TEST BOMB}

$\vec{\Xi}$
$\vec{N}$
$\dot{8}$
No
心్
$\vdots$






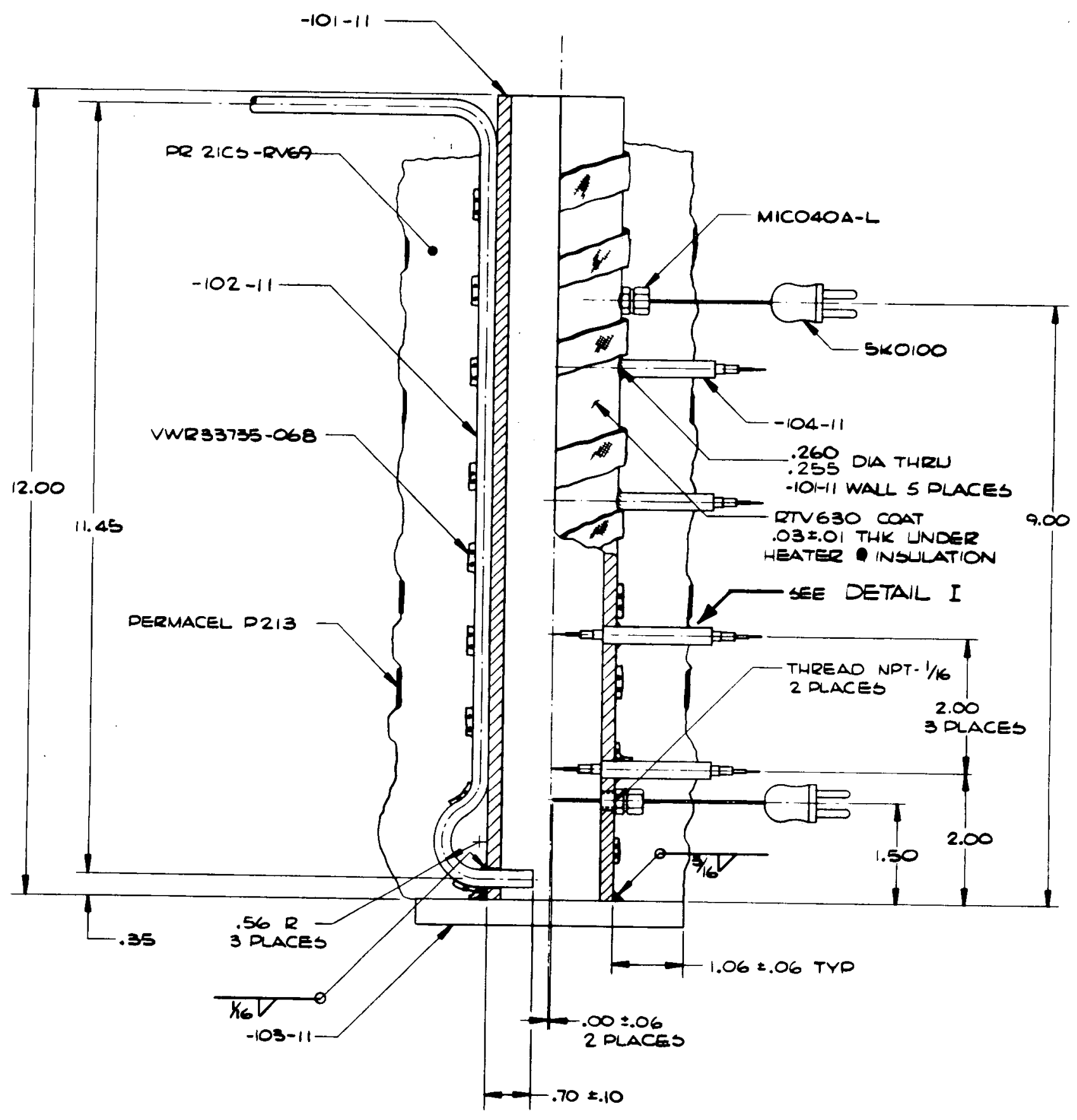

-ASSY - 301-11- 


\section{DETONATION VELOCITY TEST FIXTURES DURING ASSEMBLY}

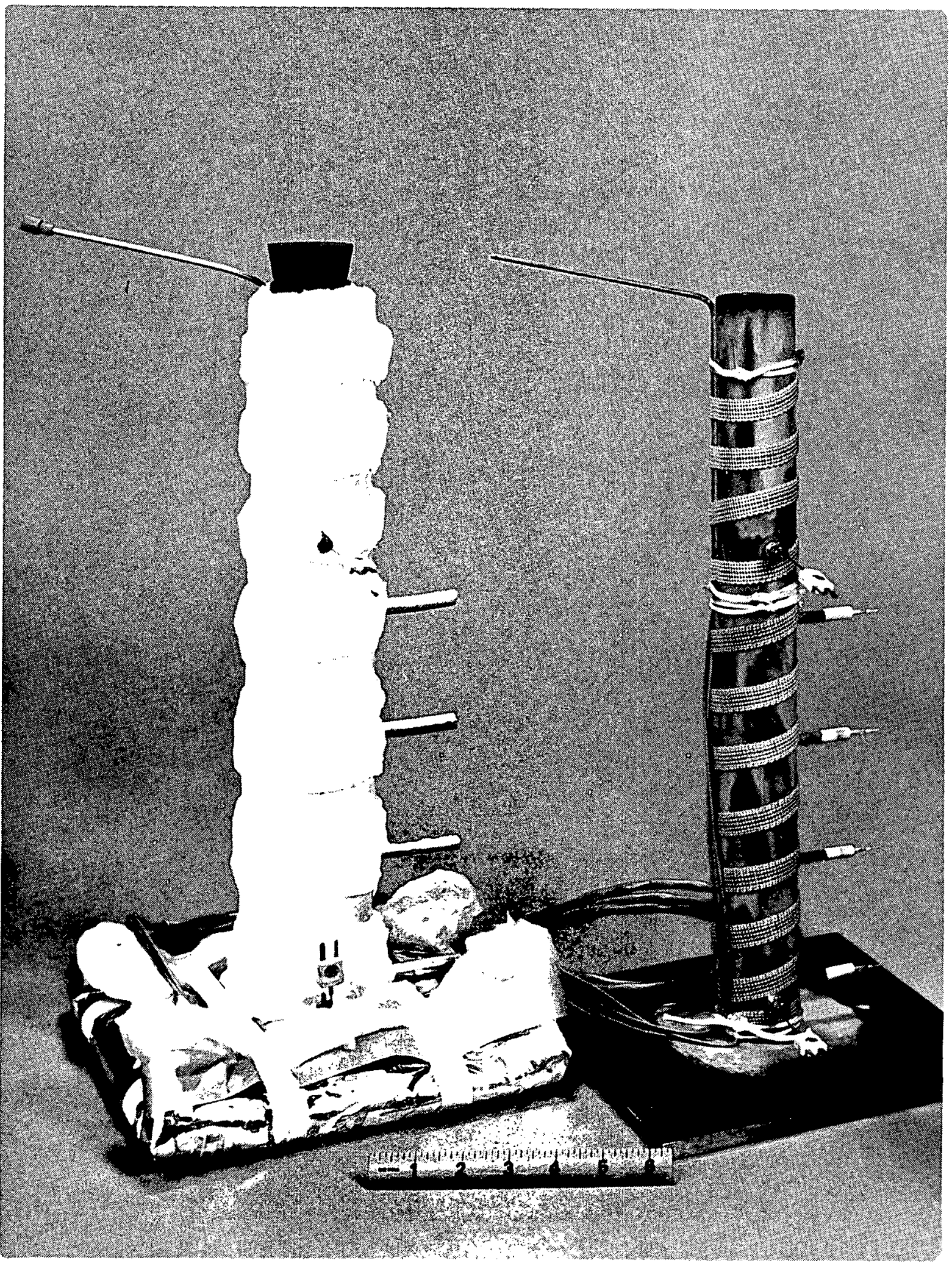




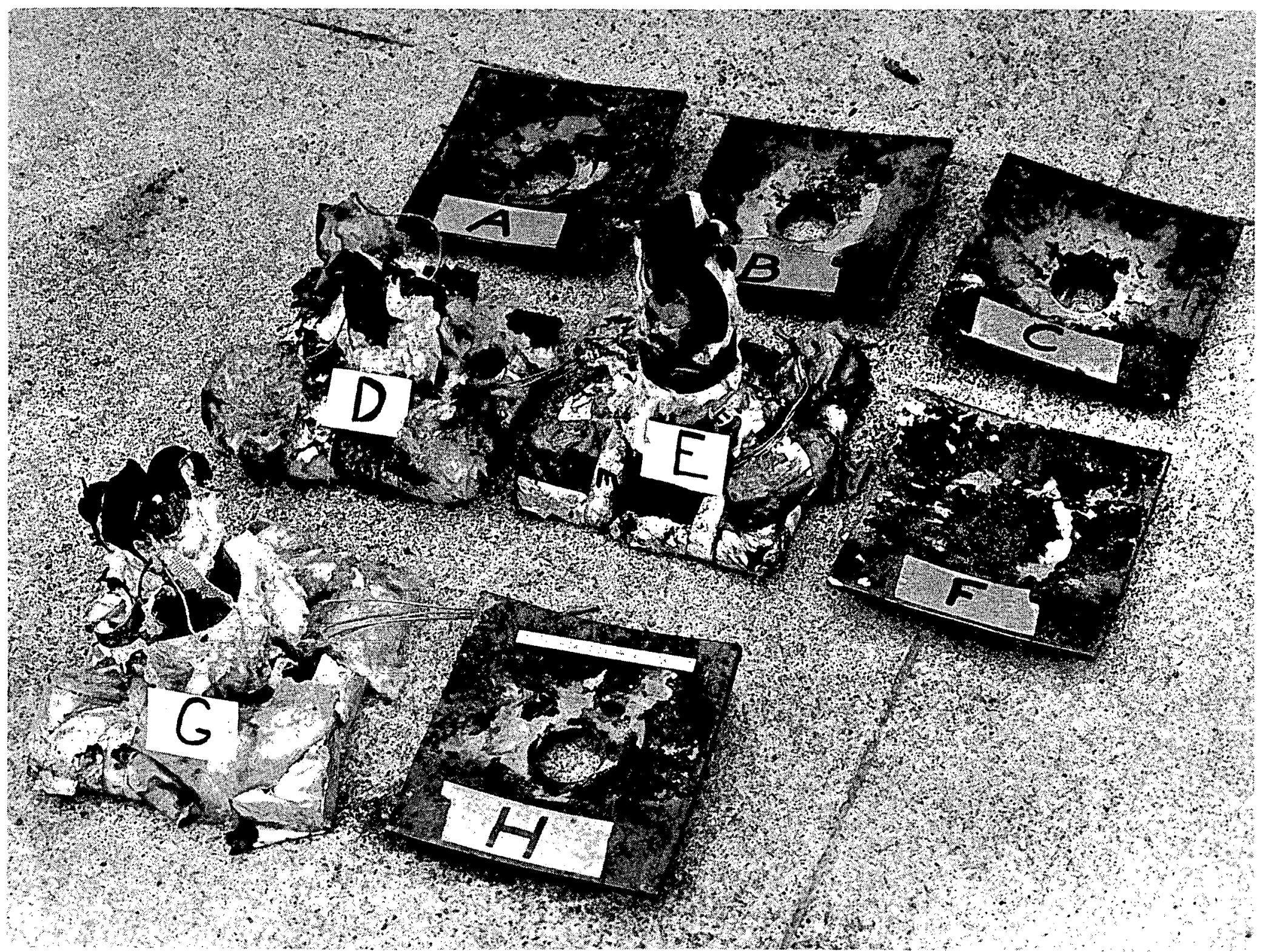


DETONATION VELOCITY TEST RESULTS

\begin{tabular}{|l|c|c|c|}
\hline \multicolumn{1}{|c|}{ Explosive Sample } & $\begin{array}{c}\text { Average } \\
\text { Detonation } \\
\text { Velocity } \\
(\mathrm{m} / \mathrm{s})\end{array}$ & $\begin{array}{c}\text { Test } \\
\text { Designation }\end{array}$ & Remarks \\
\hline (NaKCa)NO3/AcNH2 $+\mathrm{GuNO}_{3}$ & 6,096 & $\mathrm{~A}$ & Mixed OK \\
& 6,016 & $\mathrm{~B}$ & Mixing problems \\
& 6,000 & $\mathrm{C}$ & Mixed OK \\
(NaKCa)NO3/AcNH2 & 3,810 & $\mathrm{~F}$ & Mixcil OK \\
(NaKCa)NO3/Sulfolane & 2,454 \\
& 4,675 & $\mathrm{H}$ & Mixed repeatedly. \\
& 5,562 & & Detonator not fully inserted. \\
\hline
\end{tabular}




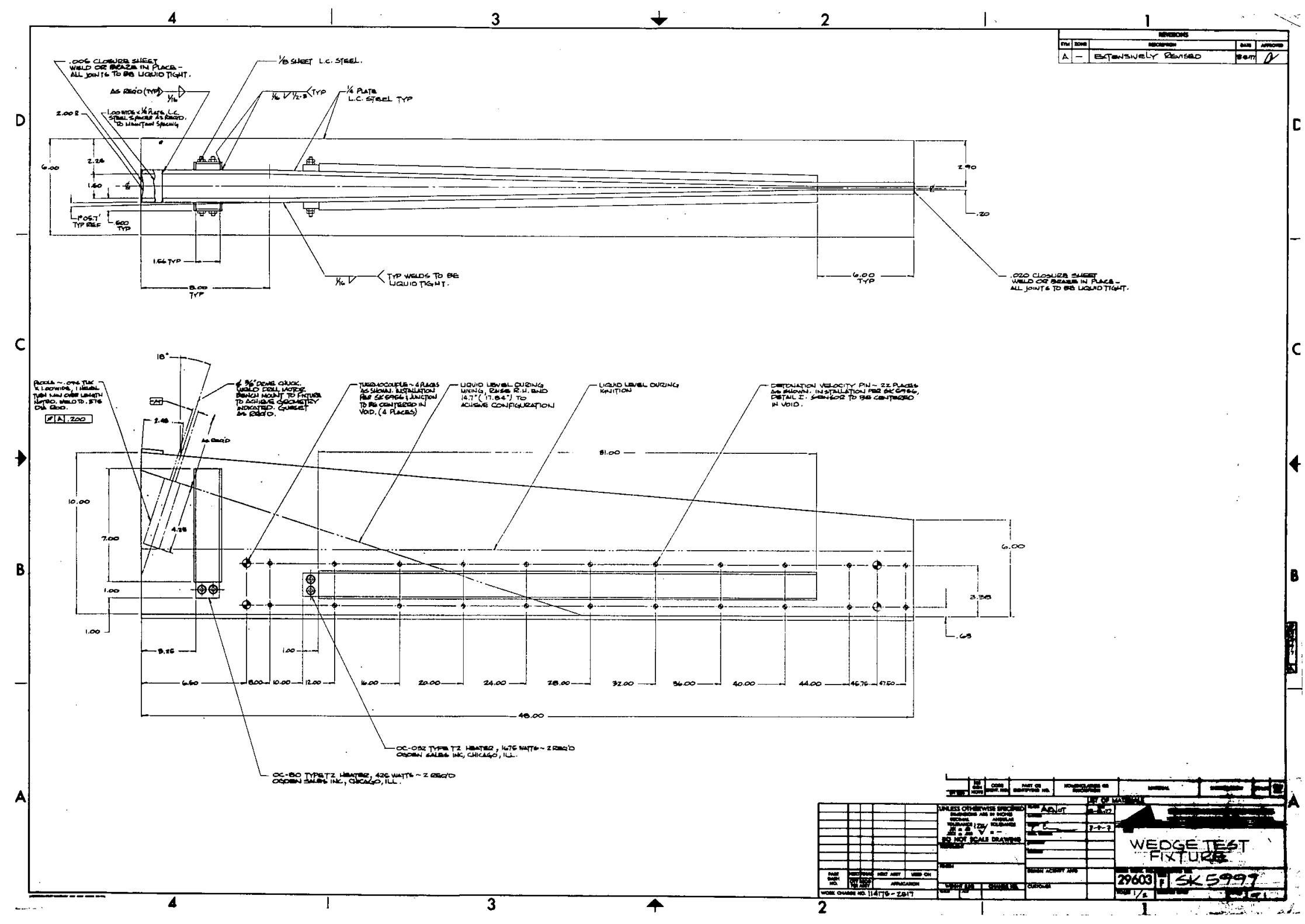


WEDGE TEST FIXTURE DURING ASSEMBLY

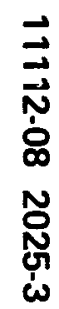

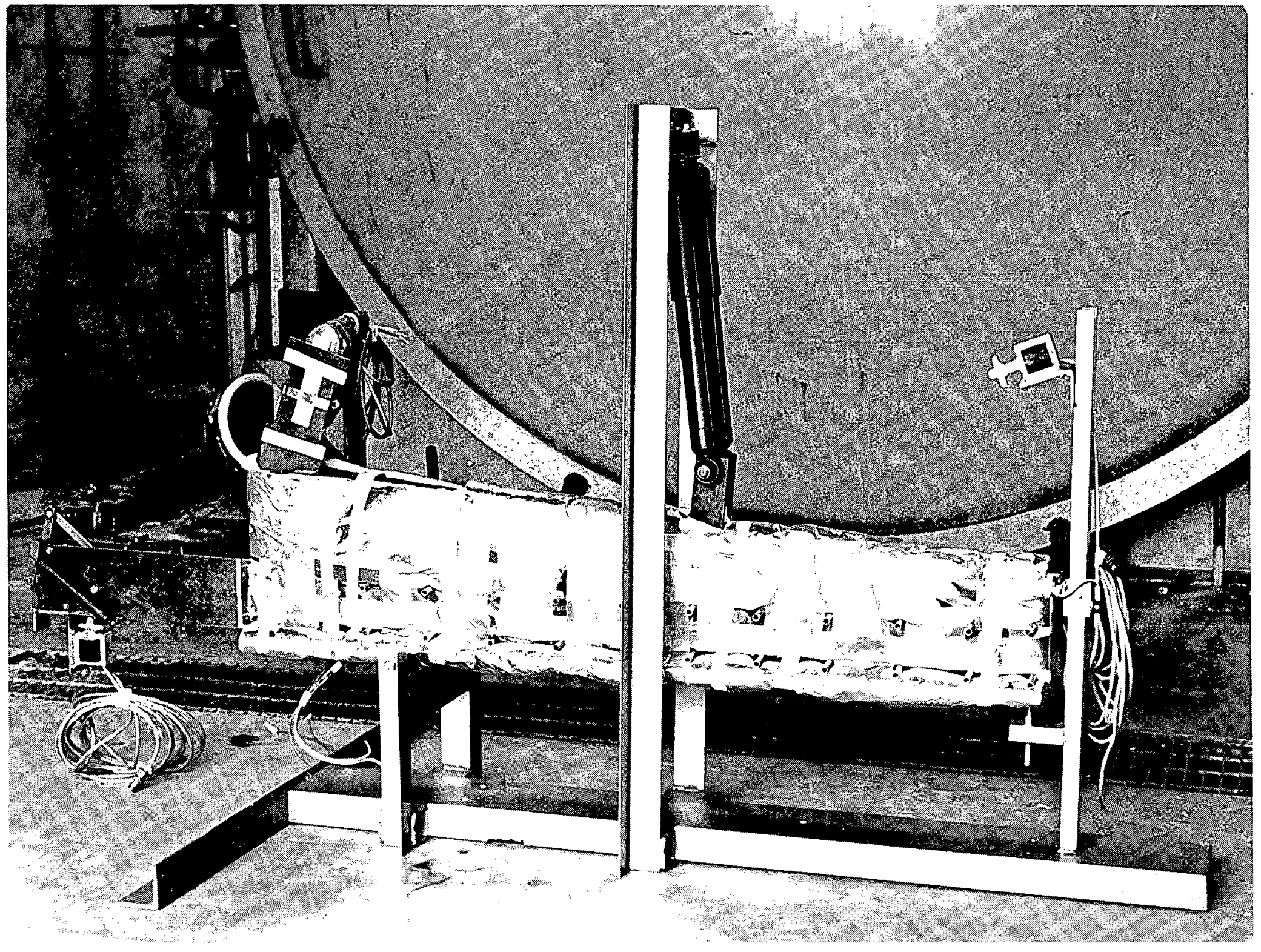


HITEX DETONATION VELOCITY AS A FUNCTION OF WEDGE GAP WIDTH

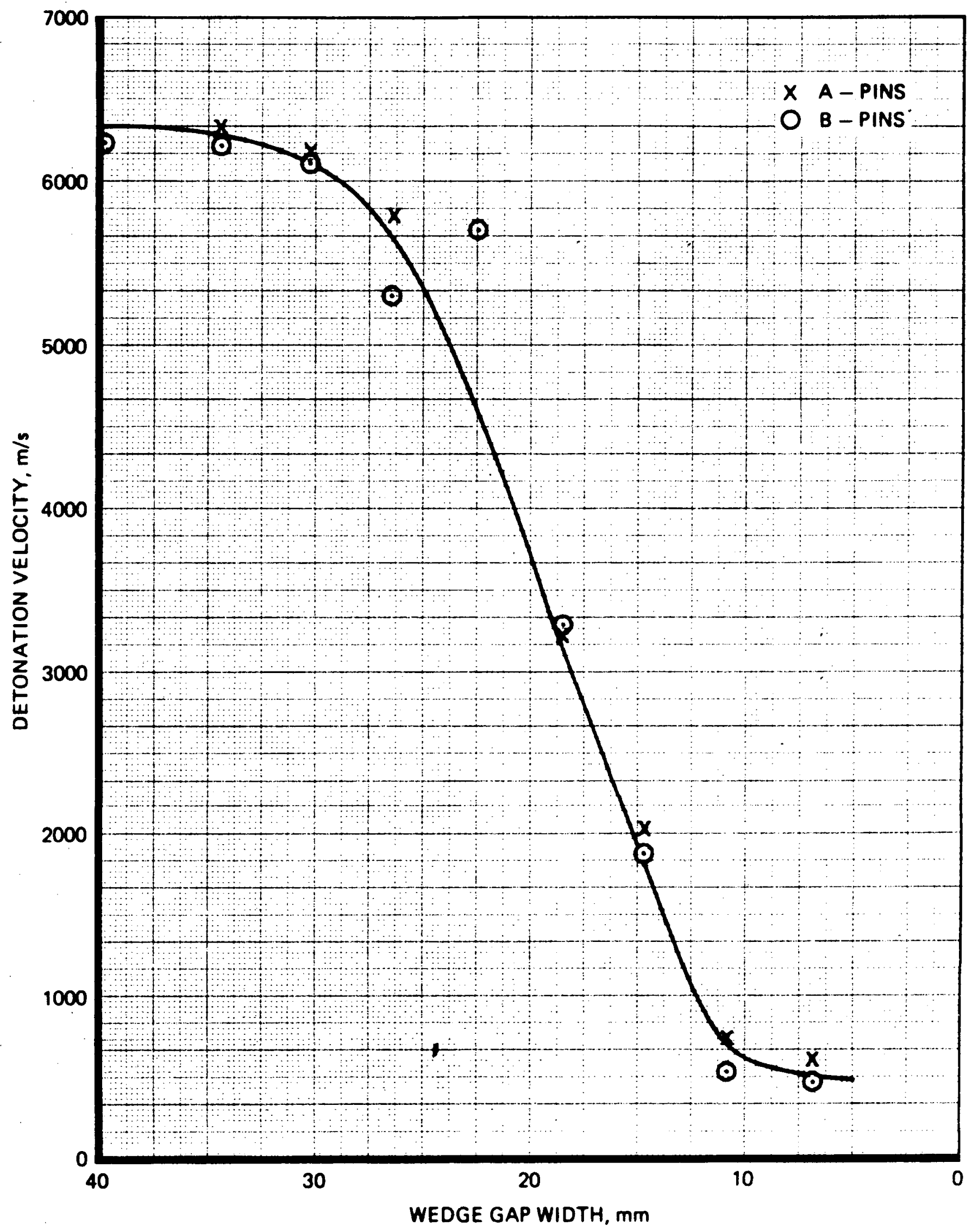




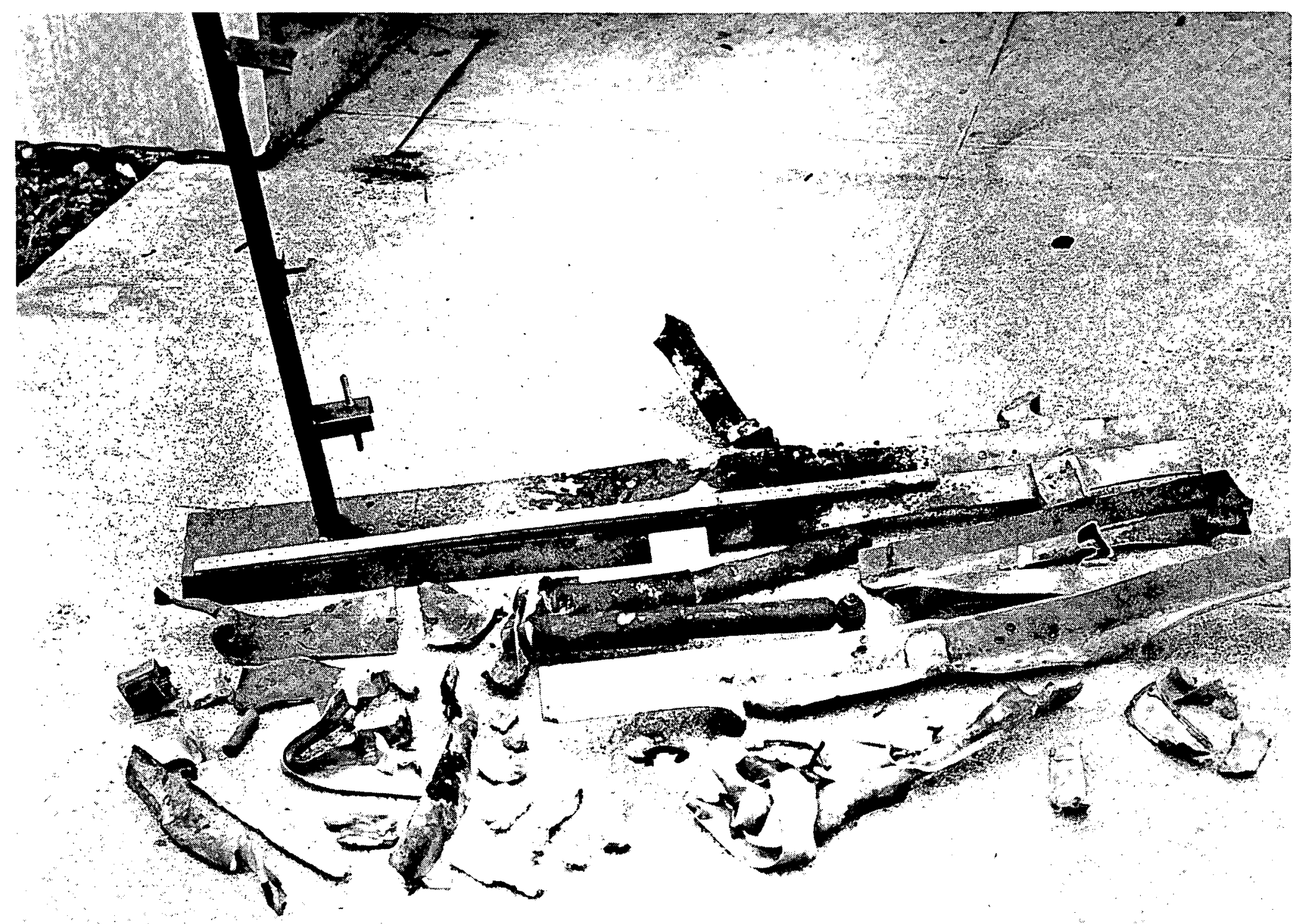


DETONATOR COOK-OFF TEST RESULTS

\begin{tabular}{|c|c|c|c|c|c|c|c|c|c|}
\hline \multirow[b]{2}{*}{ Detonator } & \multirow[b]{2}{*}{ Manufacturer } & \multirow{2}{*}{$\begin{array}{c}\text { Explosive } \\
\text { Charge, } \\
\text { g }\end{array}$} & \multirow{2}{*}{$\begin{array}{c}\text { Heating Rate } \\
\mathbf{O K}_{\mathbf{K} / \mathrm{min}}\end{array}$} & \multicolumn{2}{|c|}{$\begin{array}{c}\text { Temperature at } \\
\text { Autoignition }\end{array}$} & \multicolumn{2}{|c|}{ Temperature } & \multirow{2}{*}{$\begin{array}{c}\text { Time at } \\
\text { Temperature, } \\
\text { Minutes }\end{array}$} & \multirow{2}{*}{$\begin{array}{l}\text { Fired On } \\
\text { Command }\end{array}$} \\
\hline & & & & $\mathbf{O}_{\mathbf{K}}$ & OF & $\mathbf{O K}$ & OF & & \\
\hline E-97 & DuPont & $0.356 \mathrm{RDX}$ & 5.6 & 460 & 368 & - & - & - & $\ldots$ \\
\hline [:- -97 & DuPonl & & 3.4 & 464 & 376 & - & $\ldots$ & - & $\ldots$ \\
\hline E-97. & DuPont & & 3.6 & 466 & 379 & - & -- & $\ldots$ & $\ldots$ \\
\hline E:-97 & DuPont & & 2.7 & 461 & 371 & - & $\ldots$ & $\cdots$ & $\ldots$ \\
\hline E-97 & DuPont & & 1.1 & $\ldots$ & - & 435 & 324 & 30 & Yes \\
\hline E.97 & DuPont & & 2.3 & - & - & 454 & 358 & 30 & Yes \\
\hline Number 8 & Ilercules & & 3.7 & 420 & 297 & - & $\ldots$ & - & $\ldots$ \\
\hline MS-250-9 & DuPont & & 3.7 & 435 & 324 & $\ldots$ & - & $\ldots$ & -- \\
\hline Lead azide squib & Atlas & $\mathrm{Ph}\left(\mathrm{N}_{3}\right)_{2}$ & 3.6 & 587 & $\begin{array}{c}597 \\
(605)\end{array}$ & - & $\ldots$ & - & ..- \\
\hline$X-321-K$ & DuPont & O.52 TACOT & 4.7 & 585 & 593 & - & - & - & $\ldots$ \\
\hline
\end{tabular}




\section{HIGH-TEMPERATURE EXPLOSIVE FOR GEOTHERMAL WELL STIMULATION}

- Phase I - EXPlosive DeVElopment. CONTRACT EY-76-C-06-2336 COMPLETED

- PhASE la - EXTENDED EXPlOSIVE DEVELOPMENT. PROPOSED NOVEMBER 1977. IMMEDIATE PROGRAM START POSSIBLE.

- PHASE II - CONSTRUCTION OF AND ABOVE-GROUND TEST WITH MOBILE MIXING UNIT.

- PHASE III - LOW-PRESSURE, HIGH-TEMPERATURE GEOTHERMAL WELL DEMONSTRATION TEST

- PHASE IV - HIGH.PRESSURE, HIGH-TEMPERATURE MOBILE MIXING UNIT DEVELOPMENT AND CONSTRUCTION. 
PHASE Ia - EXTENDED HIGH-TEMPERATURE EXPLOSIVE EVALUATION FOR GEOTHERMAL WELL STIMULATION*

- COMPATIBILITY WITH MATERIALS OF CONSTRUCTION

- PHYSICAL PROPERTIES

- ENVIRONMENTAL IMPACT ASSESSMENT

- ANALYSIS OF EXPLOSIVE RESIDUES

- DETONATION VELOCITY AT HIGH PRESSURE

- FAILURE DIAMETER AT HIGH PRESSURE

- EFFECT OF STEAM OR BRINE DILUTION

- HIGH-TEMPERATURE DETONATOR/BOOSTER DEVELOPMENT

- PROgRAm Plan for field demonstration test

"RRC 78-P-826 Submitted November 11, 1977 


\section{PHASE II - DESIGN OF LOW-PRESSURE MOBILE MIXING UNIT}

- SELECT MATERIALS

- SELECT COMPONENTS

- DESIGN FLOW DIAGRAM AND CONTROLS

- ENGINEERING DRAWINGS

- PROCUREMENT OF COMPONENTS AND HARDWARE

- INSTALLATION OF TANKS, AgITATORS, PUMPS, VALVES, METERS ON MIXER/PUMPER TRAILER

- INSTALLATION OF METERING AND CONTROL EQUIPMENT IN DATA CONTROL TRAILER (DCT)

- CHECKOUT RUNS WITH INERT COMPONENTS

- EXTENDED CLOSED LOOP OPERATION WITH ONE COMPONENT AT A TIME

- SELECTION OF REMOTE TEST SITE

- INSTALLATION OF ABOVE-gROUND SIMULATED BORE HOLE AND DISPOSAL UNIT

- ABOVE-GROUND DEMONSTRATION TEST WITH INVITED OBSERVERS (POTENTIAL USERS) 


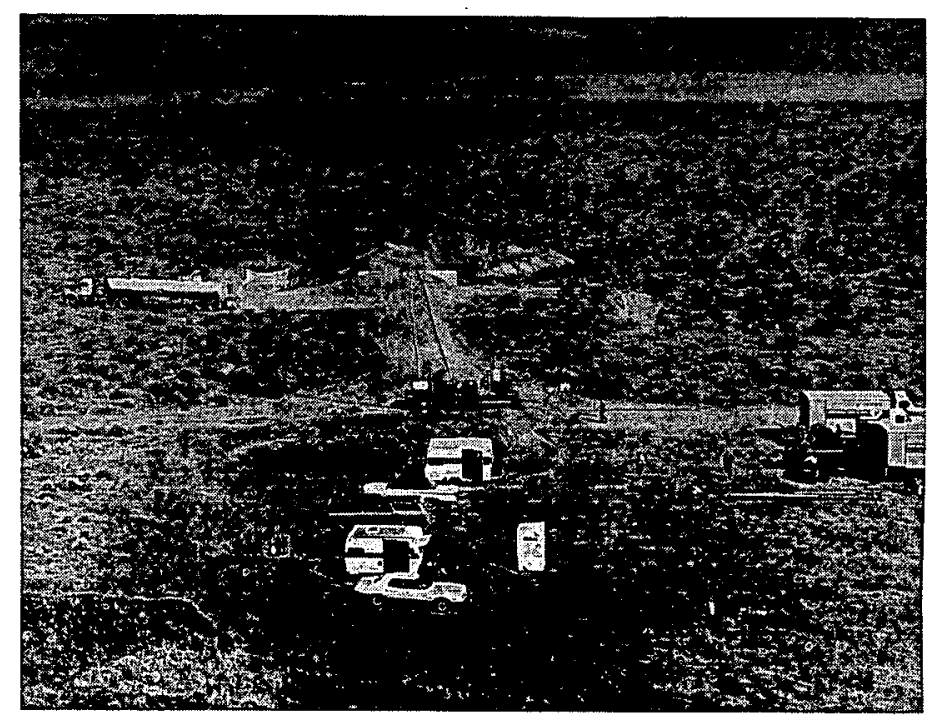

GENERAL LAYOUT

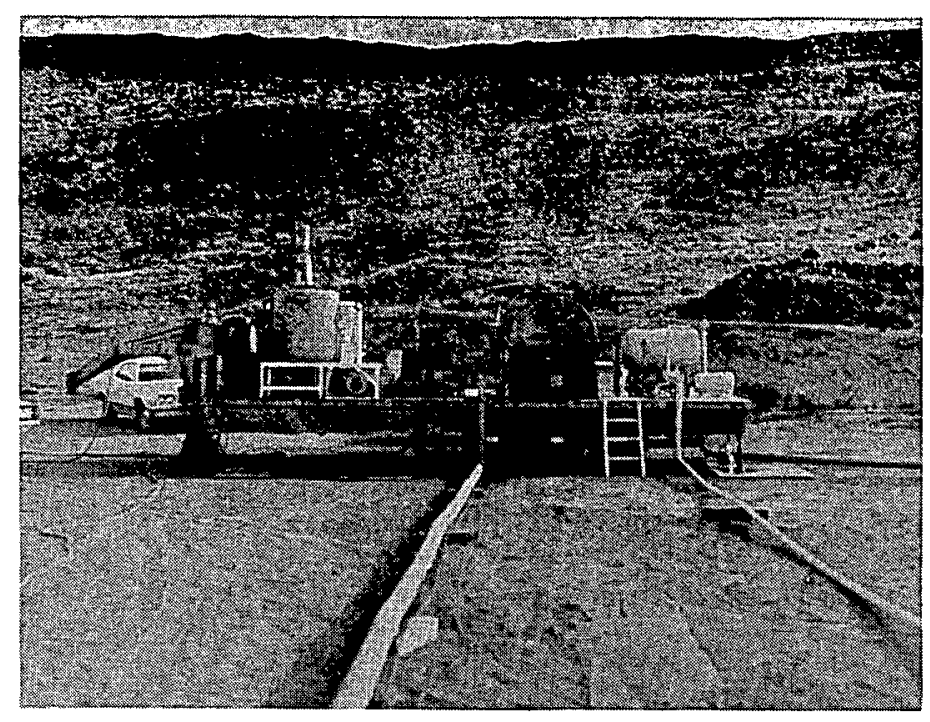

RIG AND DELIVERY LINES FOR TWO COMPONENTS

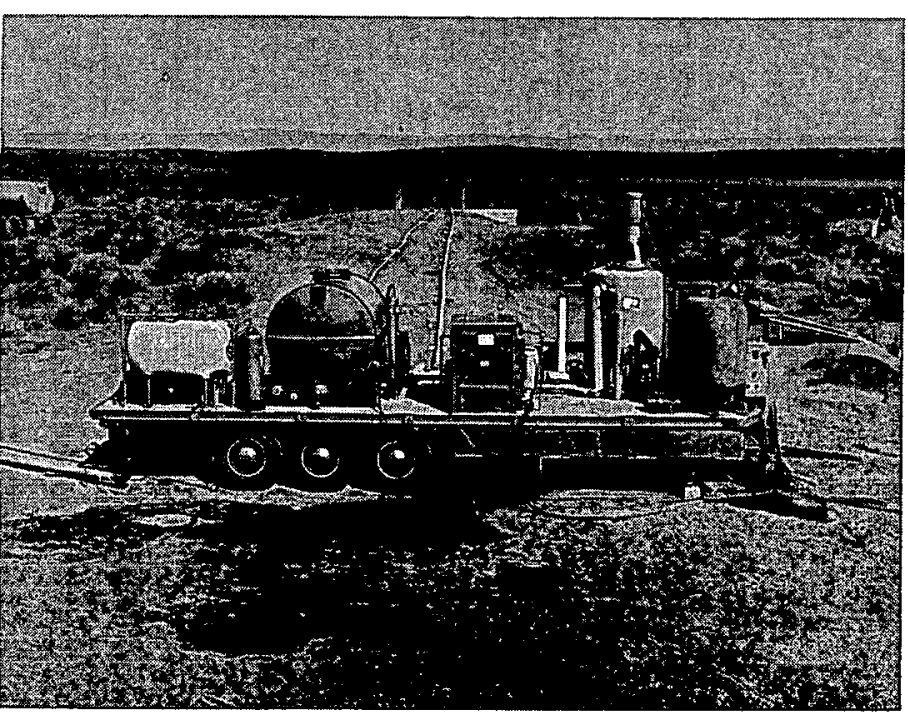

TRAILER-MOUNTED LOW PRESSURE RIG

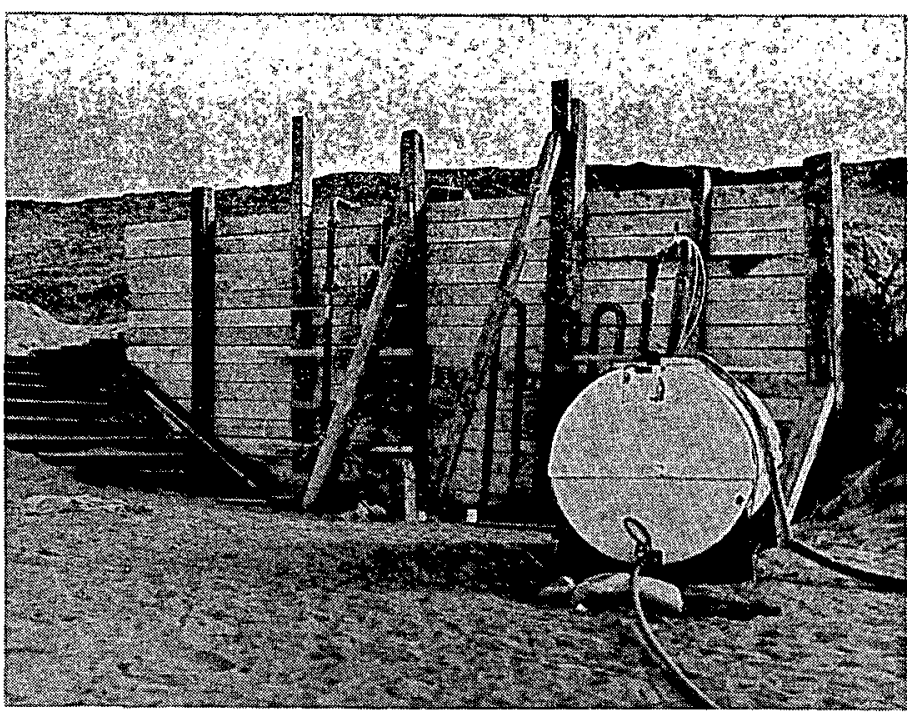

EXPLOSIVE MIXING TEST SETUP AND CATCH TANK LOCATION: ATOMIC ENERGY COMMISSION HANFORD RESERVATION WASHINGTON STATE 
PETROLEUM TECHNOLOGY CORPORATION

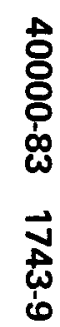

ASTRO FLOW II CHEMICAL EXPLOSIVE FRACTURING EQUIPMENT






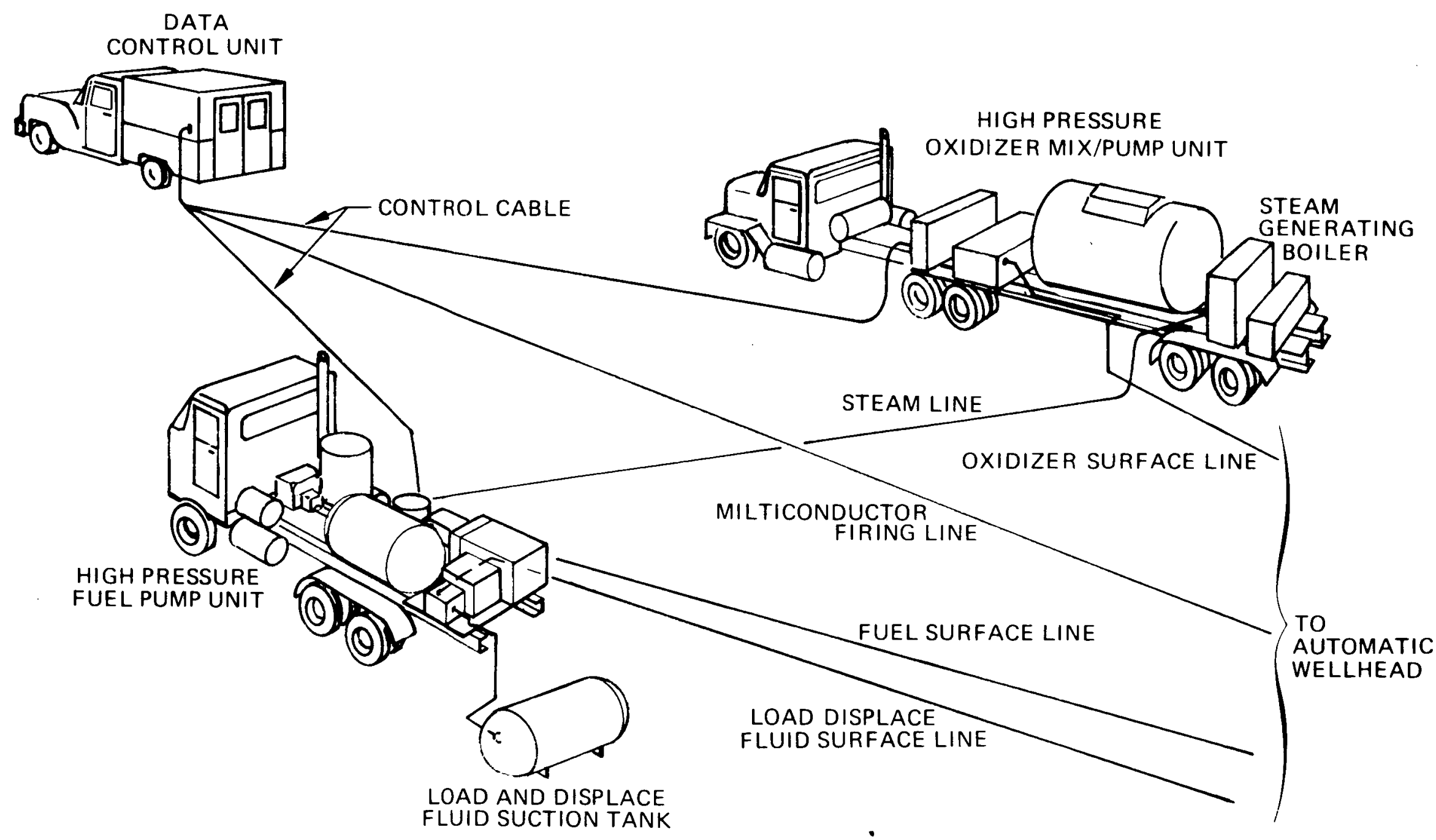

